\title{
Digamma, what next?
}

\section{Roberto Franceschini, ${ }^{a}$ Gian F. Giudice, ${ }^{a}$ Jernej F. Kamenik, ${ }^{a, b, c}$ Matthew McCullough, ${ }^{a}$ Francesco Riva, ${ }^{a}$ Alessandro Strumia ${ }^{a, d}$ and Riccardo Torre ${ }^{e}$}

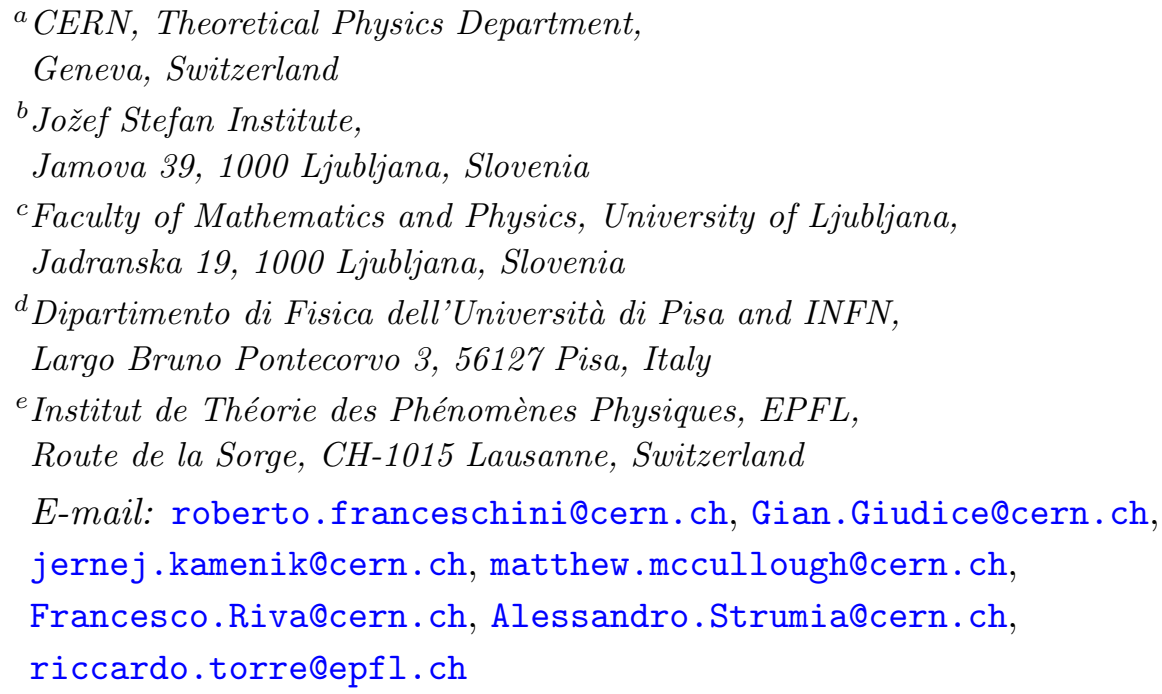

ABSTRACT: If the $750 \mathrm{GeV}$ resonance in the diphoton channel is confirmed, what are the measurements necessary to infer the properties of the new particle and understand its nature? We address this question in the framework of a single new scalar particle, called digamma $(\digamma)$. We describe it by an effective field theory, which allows us to obtain general and model-independent results, and to identify the most useful observables, whose relevance will remain also in model-by-model analyses. We derive full expressions for the leading-order processes and compute rates for higher-order decays, digamma production in association with jets, gauge or Higgs bosons, and digamma pair production. We illustrate how measurements of these higher-order processes can be used to extract couplings, quantum numbers, and properties of the new particle.

Keywords: Phenomenological Models

ArXiv EPrint: 1604.06446 


\section{Contents}

1 Introduction $\quad 2$

$2 \quad p p \rightarrow \digamma$ : single production $\quad 3$

2.1 Experimental status 3

2.2 Theoretical framework 5

3 ケ decays 9

$\begin{array}{lll}3.1 & \text { Two-body } \digamma \text { decays } & 10\end{array}$

3.2 Three-body $\digamma$ decays 13

$\begin{array}{lll}3.3 & \text { Four-body } \digamma \text { decays } & 14\end{array}$

$4 \quad p p \rightarrow \digamma j, \digamma j j:$ associated production with jets $\quad 15$

$4.1 \mathrm{CP}$ of $\digamma$ from $p p \rightarrow \digamma j j \quad 16$

$\begin{array}{lll}5 & p p \rightarrow \digamma V, \digamma h: \text { EW associated production } & 19\end{array}$

$5.1 \mathrm{CP}$ of $\digamma$ from $p p \rightarrow \digamma Z, \digamma W$

5.2 EFT expansion and associated production 24

$6 \quad p p \rightarrow \digamma \digamma$ : pair production $\quad 26$

6.1 Effective theory parametrisation 26

6.2 Model computation in low energy theorem approximation 28

6.3 Full computation beyond the LET approximation 33

6.4 Pair production of a pseudo-scalar resonance 34

6.5 Decorrelating single and pair production 35

6.6 Resonant pair production 36

6.7 Pair production phenomenology 36

$\begin{array}{lll}7 & \text { Summary } & \mathbf{3 8}\end{array}$

7.1 Identifying the weak representation 38

$\begin{array}{ll}7.2 & \text { Identifying the initial state } \\ \end{array}$

7.3 Measuring $\digamma$ couplings 40

7.4 Identifying the CP parity 40

$\begin{array}{ll}7.5 & \text { Pair production }\end{array}$

A Effective Lagrangian in the unitary gauge 42

B $\digamma$ decay widths including the mixing with the Higgs 42 


\section{Introduction}

Preliminary LHC data at $\sqrt{s}=13 \mathrm{TeV}$ show a hint for a new resonance in $p p \rightarrow \gamma \gamma$ (thereby denoted by the letter ${ }^{1}$ digamma, $\digamma$ ) at invariant mass of $750 \mathrm{GeV}[1,2]$, which stimulated intense experimental and theoretical interest. On the experimental side, dedicated analyses strengthen the statistical significance of the excess [3-6]. New measurements, which are underway, will tell us whether the excess is real and, if so, a thorough exploration of the new particle's properties will start.

Superficially, the situation looks similar to the discovery of the Higgs boson $h$, which first emerged as a peak in $\gamma \gamma$ at $125 \mathrm{GeV}$. Various computations and considerations can be readapted today from the Higgs case. However, $h$ has large couplings to SM massive vectors, unlike $\digamma$. Furthermore, in the Higgs case, the Standard Model (SM) predicted everything but the Higgs mass. Theorists made precision computations, and experimentalists made optimised measurements of Higgs properties such as its spin and parity, which did not lead to any surprise.

Today, with the digamma, we are swimming in deep water. Many key issues related to the new resonance remain obscure. Does it have spin 0,2 , or more? Is it narrow or broad? Or, more generally, how large are its couplings? To which particles can it decay? Do its couplings violate $\mathrm{CP}$ ? If not, is it $\mathrm{CP}$-even or CP-odd? Is it a weak singlet or a weak doublet or something else? Is it produced through $g g, q \bar{q}$ or weak vector collisions? Is it elementary or composite? Is it a cousin of the Higgs boson? Is it related to the mechanism of electroweak breaking or to the naturalness problem? What is its role in the world of particle physics? Who ordered that?

Answering each one of these questions could point to different theoretical directions, which at the moment look equally (im)plausible. The observation of $\gamma \gamma$ could be only the tip of an iceberg. Here we take a purely phenomenological approach. The goal of this paper is discussing and reviewing how appropriate measurements could address some of these questions.

So many possibilities are open that, not to get lost in a plethora of alternatives, we will focus on the simplest 'everybody's model'. The model involves a new scalar $\digamma$ with $M_{\digamma} \approx 750 \mathrm{GeV}$ and effective interactions to photons and other SM states. ${ }^{2}$

The paper is structured as follows. In section 2 and appendix B we describe and fit the experimental data and present the theoretical framework that can account for $p p \rightarrow \digamma \rightarrow \gamma \gamma$. In section 3 we provide full expressions (including terms suppressed

\footnotetext{
${ }^{1}$ Digamma $(\digamma)$ is a letter of the archaic Greek alphabet, originating from the Phoenician letter waw. The digamma was present in Linear B Mycenean Greek and Æolic Greek, but later disappeared from classical Greek probably before the $7^{\text {th }}$ century BC. However, it remained in use as a symbol for the number 6 , because it occupied the sixth place in the archaic Greek alphabet and because it is made of two gammas, the third letter of the Greek alphabet. As a numeral it was also called episemon during Byzantine times and stigma (as a ligature of the letters sigma and tau) since the Middle Ages. In our context, the reference to the number six is fitting, as the mass of the digamma particle is 6 , in units of the Higgs mass. Moreover, the historical precedent of the disappearance of the letter $\digamma$ is a reminder that caution is necessary in interpretations of the particle $\digamma$.

${ }^{2}$ The following list of references consider this model and its collider phenomenology [7-79]. Studies focussing explicitly on a pseudo-scalar version include [80-107].
} 


\begin{tabular}{|c|c|c|c|c|c|c|c|}
\hline \multirow[b]{2}{*}{$\sqrt{s}=13 \mathrm{TeV}$} & \multirow[b]{2}{*}{ eq. } & \multicolumn{6}{|c|}{$\digamma$ couples to } \\
\hline & & $b \bar{b}$ & $c \bar{c}$ & $s \bar{s}$ & $u \bar{u}$ & $d \bar{d}$ & $G G$ \\
\hline$\sigma_{\digamma j} / \sigma_{\digamma}$ & $(4.1 a)$ & $9.2 \%$ & $7.6 \%$ & $6.8 \%$ & $6.7 \%$ & $6.2 \%$ & $27 . \%$ \\
\hline$\sigma_{\digamma b} / \sigma_{\digamma}$ & $(4.1 b)$ & $6.2 \%$ & 0 & 0 & 0 & 0 & $0.32 \%$ \\
\hline$\sigma_{\digamma j j} / \sigma_{\digamma}$ & $(4.1 c)$ & $1.4 \%$ & $1.0 \%$ & $0.95 \%$ & $1.2 \%$ & $1.0 \%$ & $4.7 \%$ \\
\hline$\sigma_{\digamma j b} / \sigma_{\digamma}$ & $(4.1 d)$ & $1.2 \%$ & $0.18 \%$ & $0.19 \%$ & $0.34 \%$ & $0.31 \%$ & $0.096 \%$ \\
\hline$\sigma_{\digamma b b} / \sigma_{\digamma}$ & $(4.1 e)$ & $0.31 \%$ & $0.17 \%$ & $0.18 \%$ & $0.34 \%$ & $0.31 \%$ & $0.024 \%$ \\
\hline$\sigma_{\digamma \gamma} / \sigma_{\digamma}$ & $(5.1 b)$ & $0.37 \%$ & $1.5 \%$ & $0.38 \%$ & $1.6 \%$ & $0.41 \%$ & $\ll 10^{-6}$ \\
\hline$\sigma_{\digamma Z} / \sigma_{\digamma}$ & $(5.1 b)$ & $1.1 \%$ & $1.1 \%$ & $1.3 \%$ & $2.0 \%$ & $1.9 \%$ & $310^{-6}$ \\
\hline$\sigma_{\digamma W^{+}} / \sigma_{\digamma}$ & $(5.1 c)$ & $510^{-5}$ & $1.7 \%$ & $2.4 \%$ & $2.6 \%$ & $4.1 \%$ & $\ll 10^{-6}$ \\
\hline$\sigma_{\digamma W^{-}} / \sigma_{\digamma}$ & $(5.1 d)$ & $310^{-5}$ & $2.3 \%$ & $1.2 \%$ & $1.0 \%$ & $1.7 \%$ & $\ll 10^{-6}$ \\
\hline$\sigma_{\digamma h} / \sigma_{\digamma}$ & $(5.1 e)$ & $1.0 \%$ & $1.1 \%$ & $1.2 \%$ & $1.9 \%$ & $1.8 \%$ & $110^{-6}$ \\
\hline
\end{tabular}

Table 1. Predictions for the associated production of the resonance $\digamma$, assuming that it couples to different SM particles, as more precisely described by the the effective Lagrangian of eq. (2.5). For production in association with jets we assume cuts $\eta<5$ on all rapidities, $p_{T}>150 \mathrm{GeV}$ on all transverse momenta, and angular difference $\Delta R>0.4$ for all jet pairs, while for photon-associated production we impose $\eta_{\gamma}<2.5$ and $p_{T, \gamma}>10 \mathrm{GeV}$.

by powers of $v^{2} / M_{\digamma}^{2}$ ) for $\digamma$ decays into SM vectors and we study multi-body $\digamma$ decays. In section 4 we discuss $\digamma$ production together with one or more jets. In section 5 we discuss production of $\digamma$ together with EW vectors or the Higgs boson. Table 1 summarises the predictions for these cross sections. In section 6 we discuss pair production of $\digamma$. Finally, in section 7 we summarise how the above processes can be used to gather information on the main unknown properties of $\digamma$, such as its couplings, CP-parity, production mode(s), and quantum numbers.

\section{$2 \quad p p \rightarrow \digamma$ : single production}

\subsection{Experimental status}

We briefly summarise the experimental status, updating the results of [7] in light of the new $p p \rightarrow \digamma \rightarrow \gamma \gamma$ results presented at the Moriond 2016 conference [3-6], which increase the statistical significance of the excess around $m_{\gamma \gamma} \approx 750 \mathrm{GeV}$ (up to $3.9 \sigma$ in ATLAS and $3.4 \sigma$ in CMS, locally) but do not qualitatively change the main implications.

The LHC collaborations presented different analyses: we focus on the one dedicated to spin 0 searches (spin 2 searches give similar results). In figure 1 we fit the energy spectra, extracting the favoured values of the mass of the resonance, of its width and of the number of excess events. ATLAS and CMS data at $\sqrt{s}=13 \mathrm{TeV}$ are consistent among themselves. In data at $\sqrt{s}=8 \mathrm{TeV}$ the hint of an excess is too weak to extract useful information. The 

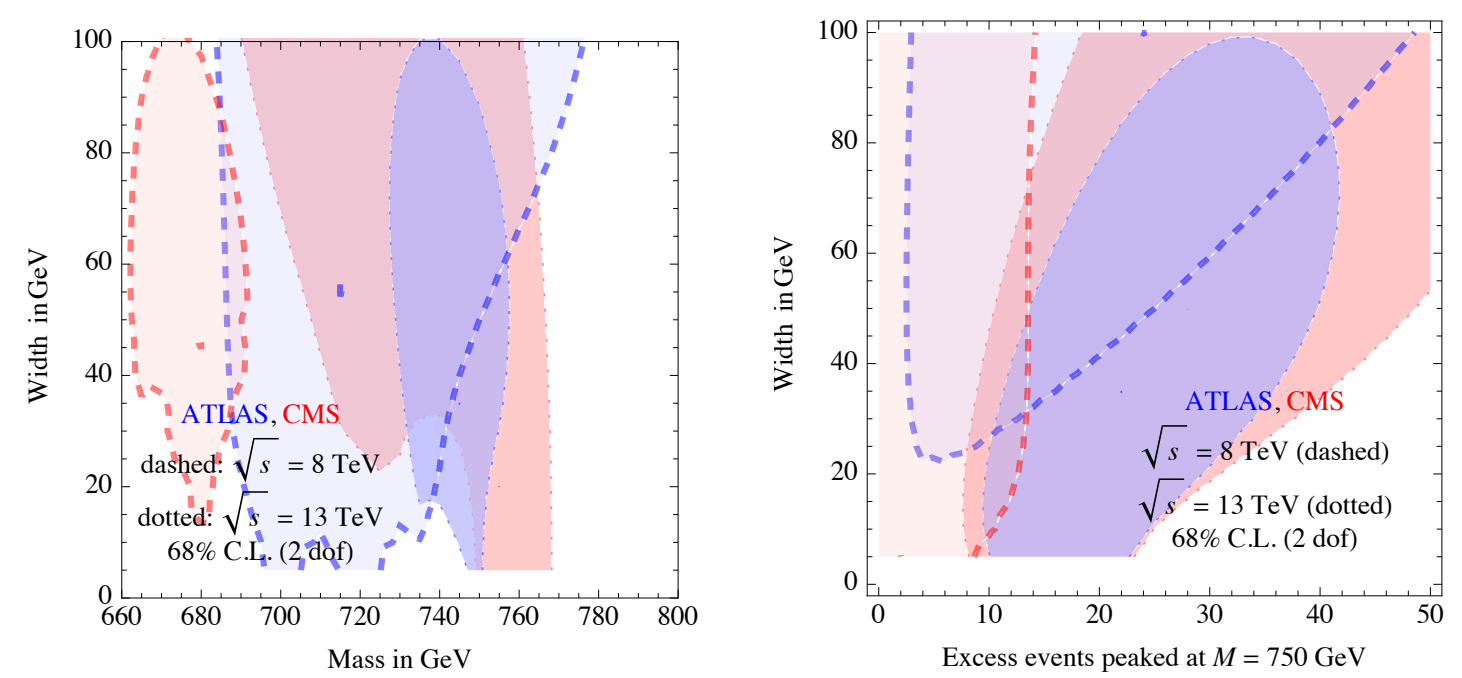

Figure 1. Fit of energy spectra obtained in spin 0 analyses. In the left plot we show the best-fit regions in the (mass, width) plane. In the right plot we fix $M_{\digamma}=750 \mathrm{GeV}$ and show the favoured values of the width and of the excess number of events.

\begin{tabular}{|c|cc|cc|}
\hline$\sigma(p p \rightarrow \gamma \gamma)$ & \multicolumn{2}{|c|}{$\sqrt{s}=8 \mathrm{TeV}$} & \multicolumn{2}{c|}{$\sqrt{s}=13 \mathrm{TeV}$} \\
& narrow & broad & narrow & broad \\
\hline CMS & $0.63 \pm 0.31 \mathrm{fb}$ & $0.99 \pm 1.05 \mathrm{fb}$ & $4.8 \pm 2.1 \mathrm{fb}$ & $7.7 \pm 4.8 \mathrm{fb}$ \\
ATLAS & $0.21 \pm 0.22 \mathrm{fb}$ & $0.88 \pm 0.46 \mathrm{fb}$ & $5.5 \pm 1.5 \mathrm{fb}$ & $7.6 \pm 1.9 \mathrm{fb}$ \\
\hline
\end{tabular}

\begin{tabular}{|c|ccc|ccc|}
\hline final & \multicolumn{3}{|c|}{$\sigma$ at $\sqrt{s}=8 \mathrm{TeV}$} & \multicolumn{3}{c|}{$\sigma$ at $\sqrt{s}=13 \mathrm{TeV}$} \\
state $f$ & observed & expected & ref. & observed & expected & ref. \\
\hline$e^{+} e^{-}, \mu^{+} \mu^{-}$ & $<1.2 \mathrm{fb}$ & $<1.2 \mathrm{fb}$ & {$[7]$} & $<5 \mathrm{fb}$ & $<5 \mathrm{fb}$ & {$[108]$} \\
$\tau^{+} \tau^{-}$ & $<12 \mathrm{fb}$ & $<15 \mathrm{fb}$ & {$[7]$} & $<60 \mathrm{fb}$ & $<67 \mathrm{fb}$ & {$[109]$} \\
$Z \gamma$ & $<11 \mathrm{fb}$ & $<11 \mathrm{fb}$ & {$[7]$} & $<28 \mathrm{fb}$ & $<40 \mathrm{fb}$ & {$[110]$} \\
$Z Z$ & $<12 \mathrm{fb}$ & $<20 \mathrm{fb}$ & {$[7]$} & $<200 \mathrm{fb}$ & $<220 \mathrm{fb}$ & {$[111]$} \\
$Z h$ & $<19 \mathrm{fb}$ & $<28 \mathrm{fb}$ & {$[7]$} & $<116 \mathrm{fb}$ & $<116 \mathrm{fb}$ & {$[112]$} \\
$h h$ & $<39 \mathrm{fb}$ & $<42 \mathrm{fb}$ & {$[7]$} & $<120 \mathrm{fb}$ & $<110 \mathrm{fb}$ & {$[113]$} \\
$W^{+} W^{-}$ & $<40 \mathrm{fb}$ & $<70 \mathrm{fb}$ & {$[7]$} & $<300 \mathrm{fb}$ & $<300 \mathrm{fb}$ & {$[114]$} \\
\hline$t \bar{t}$ & $<450 \mathrm{fb}$ & $<600 \mathrm{fb}$ & {$[7]$} & & & \\
invisible & $<0.8 \mathrm{pb}$ & - & {$[7]$} & $2.2 \mathrm{pb}$ & $1.8 \mathrm{pb}$ & {$[115]$} \\
$b \bar{b}$ & $\lesssim 1 \mathrm{pb}$ & $\lesssim 1 \mathrm{pb}$ & {$[7]$} & & & \\
$j j$ & $\lesssim 2.5 \mathrm{pb}$ & - & {$[7]$} & & & \\
\hline
\end{tabular}

Table 2. Upper box: signal rates. Lower box: bounds at $95 \%$ confidence level on $p p$ cross sections for various final states produced through a resonance with $M_{\digamma}=750 \mathrm{GeV}$ and $\Gamma / M_{\digamma} \approx 0.06$. 

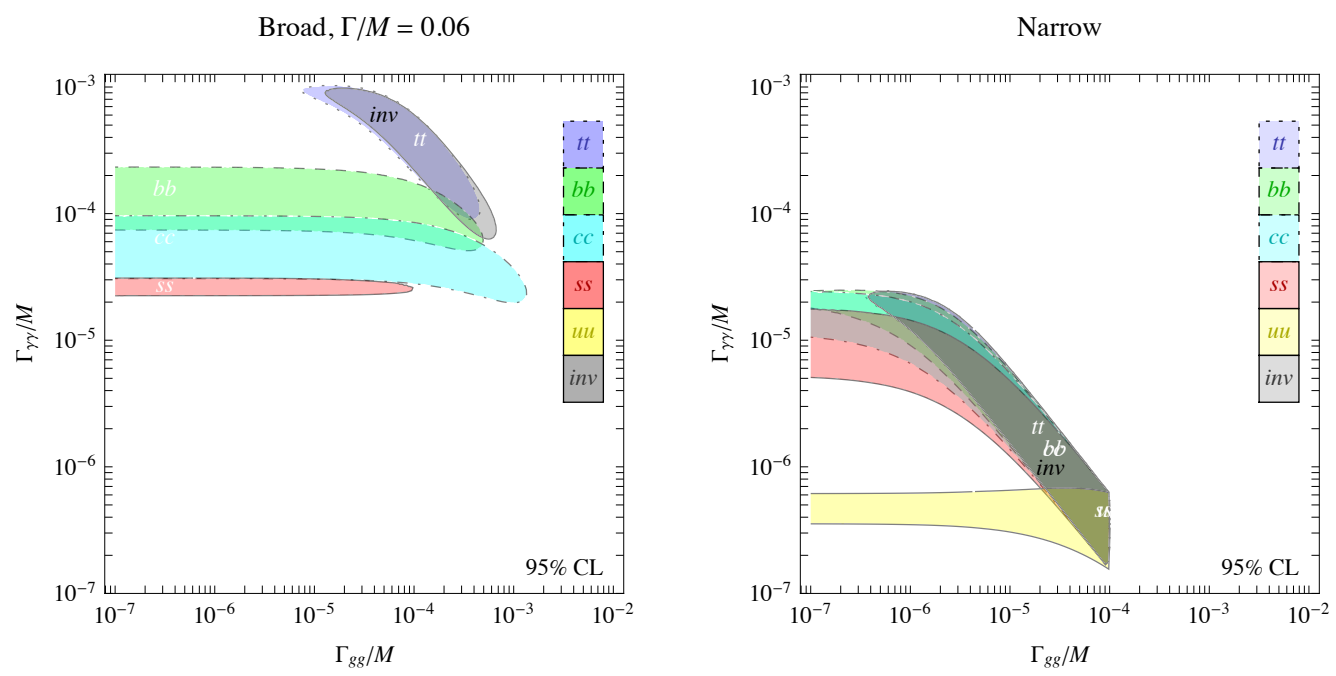

Figure 2. Global fit of $\sqrt{s}=8,13 \mathrm{TeV}$ data for the $750 \mathrm{GeV}$ excess assuming that it is due to a new resonance $\digamma$ that decays into 1 ) hypercharge vectors; 2) into gluons; 3) into a third channel considering those listed in the legend. In the left (right) panel we assume a broad (narrow) resonance.

best fit values for the excess cross section depend on both the mass and the width of the resonance, which, within statistical uncertainties, can be anything between 0 to $100 \mathrm{GeV}$. The main lesson is that it is too early to extract detailed properties from these preliminary data. We will use the reference values listed in table 2, considering the two sample cases of a narrow resonance $\left(\Gamma \ll 10 \mathrm{GeV}\right.$, which is the experimental resolution on $\left.m_{\gamma \gamma}\right)$ and a broad resonance with $\Gamma \approx 45 \mathrm{GeV}$. Table 2 also summarises the bound on other possible decay channels of the $\digamma$ resonance. In figure 2 we show the results of a global fit of signal rates and bounds for $\sqrt{s}=8$ and $13 \mathrm{TeV}$ data assuming that the $750 \mathrm{GeV}$ excess is due to a new resonance $\digamma$ that decays into: 1 ) hypercharge vectors; 2) gluons; 3 ) a third channel which could be $t \bar{t}, b \bar{b}, c \bar{c}, u \bar{u}$, or invisible particles (such as Dark Matter or neutrinos). In the left (right) panel we assume a broad (narrow) resonance. A message that can be indirectly read from figure 2 is that production from gluons or from heavy quarks remains mildly favoured with respect to production from photons [73-79] or light quarks, which predict a too small $13 \mathrm{TeV} / 8 \mathrm{TeV}$ cross section ratio.

\subsection{Theoretical framework}

The cross section for single production of a scalar $\digamma, \sigma_{\digamma}=\sigma(p p \rightarrow \digamma)$, can be written in the narrow-width approximation in terms of its decay widths into partons $\wp, \Gamma_{\wp}=\Gamma(\digamma \rightarrow$

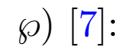

$$
\sigma(p p \rightarrow \digamma)=\frac{1}{s} \sum_{\wp} C_{\wp} \frac{\Gamma_{\wp}}{M_{\digamma}}
$$


Here we extend the list of parton luminosity factors $C_{\wp}$ given in [7] by including massive SM vectors, which can be either Transverse or Longitudinal $^{3}$

\begin{tabular}{|c|cccccccccccc|}
\hline$\sqrt{s}$ & $C_{b \bar{b}}$ & $C_{c \bar{c}} C_{s \bar{s}} C_{d \bar{d}} C_{u \bar{u}}$ & $C_{g g}$ & $C_{\gamma \gamma}$ & $C_{Z_{L} Z_{L}}$ & $C_{Z_{T} Z_{T}} C_{Z_{T} \gamma} C_{W_{L} W_{L}} C_{W_{T} W_{T}}$ \\
\hline $8 \mathrm{TeV}$ & 1.07 & 2.7 & 7.2 & 89 & 158 & 174 & $11(8)$ & 0.01 & 0.3 & 3.1 & 0.03 & 0.8 \\
$13 \mathrm{TeV}$ & 15.3 & 36 & 83 & 627 & 1054 & 2137 & $54(64)$ & 0.14 & 2.8 & 27 & 0.4 & 8 \\
\hline
\end{tabular}

The gauge boson parton luminosity functions in the table are obtained convoluting the $W_{L, T}, Z_{L, T}$, and photon leading order splitting functions with the quark pdfs ("NNPDF30_1o_as_0118" set [116]), evaluated at factorisation scale $\mu_{W}=M_{W}, \mu_{Z}=M_{Z}$ and $\mu_{\gamma}=10 \mathrm{GeV}$. The two numbers for the $C_{\gamma \gamma}$ correspond to the photon luminosities obtained using the photon pdfs in the "NNPDF30_1o_as_0118" set (outside parentheses) and the number obtained with the aforementioned procedure (inside parentheses). These numbers come with a significant uncertainty, due to the sensitivity on the aforementioned choice of renormalisation scale. We have checked that they are able to reproduce, within a factor of two, the relevant processes computed with MadGraph5 [117]. We consider this precision sufficient for our study, but we stress that going to higher order splitting functions for the gauge bosons can make this error smaller, which may be needed in the future. From the table above we see that the $C$-factors for longitudinal vector bosons are highly suppressed. Longitudinal vector boson fusion (VBF) can never become relevant compared to photon-fusion, and can therefore be neglected. The situation is different for the transverse VBF, which can give a sizeable contribution to the total production.

From eq. (2.1) we obtain, at $\sqrt{s}=13 \mathrm{TeV}$

$$
\begin{aligned}
\sigma(p p & \rightarrow \digamma)=\left[4900 \frac{\Gamma_{g g}}{M_{\digamma}}+2400 \frac{\Gamma_{u \bar{u}}}{M_{\digamma}}+1400 \frac{\Gamma_{d \bar{d}}}{M_{\digamma}}+190 \frac{\Gamma_{s \bar{s}}}{M_{\digamma}}+83 \frac{\Gamma_{c \bar{c}}}{M_{\digamma}}+35 \frac{\Gamma_{b \bar{b}}}{M_{\digamma}}+\right. \\
& \left.+150 \frac{\Gamma_{\gamma \gamma}}{M_{\digamma}}+62 \frac{\Gamma_{Z \gamma}}{M_{\digamma}}+18 \frac{\Gamma_{W_{T} W_{T}}}{M_{\digamma}}+0.92 \frac{\Gamma_{W_{L} W_{L}}}{M_{\digamma}}+6.5 \frac{\Gamma_{Z_{T} Z_{T}}}{M_{\digamma}}+0.32 \frac{\Gamma_{Z_{L} Z_{L}}}{M_{\digamma}}\right] \mathrm{pb} .
\end{aligned}
$$

We do not consider production from a loop of $t$ quarks because it cannot reproduce the diphoton excess without predicting, at the same time, a $\Gamma(F \rightarrow t \bar{t})$ above the bound in table 2. Assuming that $\digamma$ decay to a single parton channel saturates the $\digamma$ decay width at $\Gamma / M_{\digamma} \simeq 0.06$ implies $\mathrm{BR}(\digamma \rightarrow \gamma \gamma) \approx\{0.018,0.70,1.6,3.8\} \times 10^{-3}$ for $\wp=\{g g, \bar{s} s, \bar{c} c, \bar{b} b\}$ in order to reproduce the observed $\sigma(p p \rightarrow \digamma \rightarrow \gamma \gamma)$.

In eq. (2.2) we omitted QCD $K$-factors describing higher order corrections, since they are not known for all channels. In the case of the gluons and quarks contributions they are given at NLO by $K_{g g} \simeq 1.5$ and $K_{q \bar{q}} \simeq 1.2$ (see for instance $[7,118]$ ). In the rest of the paper we will systematically avoid including any $K$ factor, since they are not known for the majority of the processes we consider.

Effective Lagrangian up to dimension 5. While the above framework captures the physics of the simplest $p p \rightarrow \digamma$ process, a more systematic parametrisation is needed to

\footnotetext{
${ }^{3}$ We omit mixed $L T$ contributions since they are suppressed by an additional power of $M_{W, Z}^{2} / M_{\digamma}^{2}$, see eq. (B.6).
} 
describe $\digamma$ production in association with other SM particles. This can be done with an Effective Field Theory (EFT) approach, ${ }^{4}$ which also provides an ideal language to match to explicit microscopic models. We assume that the underlying theory is broadly characterised by a mass scale $\Lambda$ and that the light degrees of freedom are the SM fields and $\digamma$, so that $M_{\digamma} \ll \Lambda$. The renormalisable interactions of $\digamma$ are encoded in the Lagrangian

$$
\mathscr{L}_{4}=\mathscr{L}_{\mathrm{SM}}+\frac{\left(\partial_{\mu} \digamma\right)^{2}}{2}-V(\digamma, H)
$$

where $\mathscr{L}_{\mathrm{SM}}$ is the SM part, while the scalar potential can be written as ${ }^{5}$

$$
V(\digamma, H)=\frac{m_{\digamma}^{2}}{2} \digamma^{2}+\kappa_{\digamma} m_{\digamma} \digamma^{3}+\lambda_{\digamma} \digamma^{4}+\kappa_{\digamma H} m_{\digamma} \digamma\left(|H|^{2}-v^{2}\right)+\lambda_{\digamma H} \digamma^{2}\left(|H|^{2}-v^{2}\right),
$$

with generic couplings $\kappa_{\digamma, \digamma H}$ and $\lambda_{\digamma, \digamma H}$. A possible tadpole term in eq. (2.4) can be eliminated with a shift of $\digamma$, while we have absorbed an EWSB contribution from $\lambda_{\digamma H}$ to the $\digamma$ mass into a redefinition of eq. (2.3). The mass eigenstate $M_{\digamma}$ is slightly different from the mass parameter $m_{\digamma}$, as discussed in appendix B.

For $\Lambda$ in the $\mathrm{TeV}$ range, the leading non-renormalisable interactions between $\digamma$ and the SM are phenomenologically important, as we will discuss later. In full generality, the dimension-5 effective Lagrangian can be written as ${ }^{6}$

$$
\begin{aligned}
\mathscr{L}_{5}^{\text {even }}= & \frac{\digamma}{\Lambda}\left[c_{g g} \frac{g_{3}^{2}}{2} G_{\mu \nu}^{a} G^{a \mu \nu}+c_{W W} \frac{g_{2}^{2}}{2} W_{\mu \nu}^{a} W^{a \mu \nu}+c_{B B} \frac{g_{1}^{2}}{2} B_{\mu \nu} B^{\mu \nu}+c_{\psi}\left(H \bar{\psi}_{L} \psi_{R}+\text { h.c. }\right)\right. \\
& \left.+c_{H}\left|D_{\mu} H\right|^{2}-c_{H}^{\prime}\left(|H|^{4}-v^{4}\right)\right]+\frac{c_{\digamma 3}}{\Lambda} \frac{\digamma\left(\partial_{\mu} \digamma\right)^{2}}{2}
\end{aligned}
$$

for CP-even $\digamma$. In the CP-odd case, we find

$$
\mathscr{L}_{5}^{\text {odd }}=\frac{\digamma}{\Lambda}\left[\tilde{c}_{g g} \frac{g_{3}^{2}}{2} G_{\mu \nu}^{a} \tilde{G}^{a \mu \nu}+\tilde{c}_{W W} \frac{g_{2}^{2}}{2} W_{\mu \nu}^{a} \tilde{W}^{a \mu \nu}+\tilde{c}_{B B} \frac{g_{1}^{2}}{2} B_{\mu \nu} \tilde{B}^{\mu \nu}+\tilde{c}_{\psi}\left(i H \bar{\psi}_{L} \psi_{R}+\text { h.c. }\right)\right],
$$

while both structures can co-exist if CP is explicitly broken by $\digamma$ interactions. Here $\tilde{X}_{\mu \nu}=$ $\frac{1}{2} \epsilon_{\mu \nu \alpha \beta} X_{\alpha \beta}$. The real coefficients $c_{i} \equiv c_{i}^{(5)}$ involve different powers of couplings in the underlying theory and, for most of our discussion, can be taken arbitrary. Field redefinitions of the form $\psi \rightarrow \psi\left(1+c_{1} \digamma / \Lambda\right), H \rightarrow H\left(1+c_{2} \digamma / \Lambda\right)$ and $\digamma \rightarrow\left(\digamma+c_{3} \digamma^{2} / \Lambda+c_{4}|H|^{2} / \Lambda\right)$ leave the leading Lagrangian eq. (2.3) unaltered and the freedom of the coefficients $c_{1-4}$ can be used to eliminate four combinations of higher-dimensional operators proportional to the (leading) equations of motion (see [122] for a discussion in this context). Using these redefinitions we have eliminated from eq. (2.5) the structures $i \digamma \bar{\psi} \not D \psi+$ h.c., $\digamma^{3}|H|^{2}$,

\footnotetext{
${ }^{4}$ See also [21] for an alternative parametrisation of resonant di-photon phenomenology.

${ }^{5}$ For $\kappa_{\digamma}=\kappa_{\digamma H}=0$ the Lagrangian acquires a $Z_{2}$ symmetry $\digamma \rightarrow-\digamma$ that might or might not be identified with $\mathrm{CP}$, depending on the higher order interactions of $\digamma$.

${ }^{6} \mathrm{It}$ is interesting to note that the anomalous dimensions of the operators in eq. (2.5) exhibit a peculiar structure with several vanishing entries [119-121]. In particular, the $\digamma V V$ structure only renormalises the $\digamma H \bar{\psi}_{L} \psi_{R}$ and $\digamma|H|^{4}$ operators, while the $\digamma H \bar{\psi}_{L} \psi_{R}$ operators only induce $\digamma|H|^{4}$. This implies that, for instance, if some selection rule forbids the $c_{H}$ structure in the UV, then Renormalisation Group Effects, from the scale $\Lambda$ to the energy at which these interactions are used, will not generate it.
} 
$\digamma H^{\dagger} D^{2} H+$ h.c. and $\digamma^{5}$. An equivalent choice, adopted in [122], is to eliminate from $\mathscr{L}_{5}$ all operators involving derivatives. For our purposes, our choice is preferable because it allows for a more direct matching of the operators in eq. (2.5) to explicit models [7].

With this notation, eq. (2.2) for the $13 \mathrm{TeV}$ single $\digamma$ production takes the form

$$
\begin{aligned}
\sigma(p p \rightarrow \digamma)= & \frac{\mathrm{TeV}^{2}}{\Lambda^{2}}\left[613 c_{g g}^{2}+7.6 c_{u}^{2}+4.7 c_{d}^{2}+0.44 c_{s}^{2}+0.30 c_{c}^{2}+0.13 c_{b}^{2}+\right. \\
& \left.+0.01 c_{H}^{2}+0.02 c_{B B}^{2}+0.007 c_{B B} c_{W W}+0.13 c_{W W}^{2}\right] \mathrm{pb}
\end{aligned}
$$

where the only interference between the contributions of different operators concerns $c_{B B}$ and $c_{W W}$. CP-odd terms proportional to $\tilde{c}_{i}$ contribute the same amount to the cross section as their $\mathrm{CP}$ even counterparts $c_{i}$.

An important observation is that the couplings $c_{\psi}$ have a non-trivial flavour structure $[10,40]$, and can be regarded as spurions transforming as $(3, \overline{3})$ under the $\mathrm{SU}(3)_{L} \otimes$ $\mathrm{SU}(3)_{R}$ flavour symmetry. If the matrices $c_{\psi}$ are not aligned with the Yukawa couplings, $F$ mediates flavour-changing neutral currents via four-fermion interactions given by

$$
\frac{v^{2}}{\Lambda^{2} M_{\digamma}^{2}}\left(c_{\psi i j} \bar{\psi}_{L}^{i} \psi_{R}^{j}+c_{\psi j i}^{*} \bar{\psi}_{R}^{i} \psi_{L}^{j}\right)^{2}
$$

In table 3 we list the most stringent bounds on off-diagonal elements of the couplings $c_{\psi}$, evaluated in the quark mass eigenbasis. We see that off-diagonal elements must be smaller than $10^{-(3 \div 4)}(\Lambda / \mathrm{TeV})$, while at least one diagonal element must be of order unity to obtain a sizeable $\digamma$ production cross section, as can be derived from eq. (2.7). Since this seems to correspond to a fine tuning of parameters, we conclude that $\digamma$ production from quark initial states is not compatible with a generic flavour structure.

There are ways to circumvent the problem. One way is to embed $\digamma$ in a weak doublet which gives mass to down-type quarks only, while the EW vev resides primarily in the SMlike Higgs doublet, which gives mass to up-type quarks. Different solutions, more relevant in our context, can be found for a singlet $\digamma$. This can be done $[10,40]$ with appropriate flavour symmetries, alignment mechanisms, or by imposing a condition of minimal flavour violation (MFV) [123], which implies that $c_{\psi}$ is a matrix proportional to the corresponding SM Yukawa couplings. Consider first the case of only $c_{u} H \bar{q}_{L} u_{R}$ with $c_{u}$ proportional to the up-type Yukawa matrix, where the $\digamma$ production is dominated by the light quarks. In this case the coupling to top quarks is large, leading to an unacceptable decay width in $\digamma \rightarrow \bar{t} t$. More interesting is the case of couplings to down-type quarks, $c_{d} H \bar{q}_{L} d_{R}$. The Yukawa structure implies that the dominant $\digamma$ coupling is to bottom quarks, while flavour violations are kept under control either by an approximate MFV or by a flavour symmetry of the underlying theory. So, while $\digamma$ production from $\bar{c} c, \bar{s} s$, or light quarks can be obtained with special flavour structures, the case of $\bar{b} b$ can be more easily justified under the MFV assumption or with the implementation of an appropriate flavour symmetry.

Effective Lagrangian: dimension 6. It is instructive to extend our analysis of interactions between $\digamma$ and the SM to the next order in the $1 / \Lambda$ expansion: at dimension- 6 the 


\begin{tabular}{|c|c|}
\hline Observable & Bound \\
\hline$\Delta m_{K}$ & $\sqrt{\left|\operatorname{Re}\left(c_{s d}^{2}+c_{d s}^{* 2}-8.9 c_{s d} c_{d s}^{*}\right)\right|}<1.1 \times 10^{-3}(\Lambda / \mathrm{TeV})$ \\
$\epsilon_{K}$ & $\sqrt{\left|\operatorname{Im}\left(c_{s d}^{2}+c_{d s}^{* 2}-8.9 c_{s d} c_{d s}^{*}\right)\right|}<2.8 \times 10^{-5}(\Lambda / \mathrm{TeV})$ \\
$\Delta m_{D}$ & $\sqrt{\left|\operatorname{Re}\left(c_{c u}^{2}+c_{u c}^{* 2}-7.0 c_{c u} c_{u c}^{*}\right)\right|}<2.7 \times 10^{-3}(\Lambda / \mathrm{TeV})$ \\
$|q / p|, \phi_{D}$ & $\sqrt{\left|\operatorname{Im}\left(c_{c u}^{2}+c_{u c}^{* 2}-7.0 c_{c u} c_{u c}^{*}\right)\right|}<3.2 \times 10^{-4}(\Lambda / \mathrm{TeV})$ \\
$\Delta m_{B_{d}}$ & $\sqrt{\left|\operatorname{Re}\left(c_{b d}^{2}+c_{d b}^{* 2}-6.3 c_{b d} c_{d b}^{*}\right)\right|}<3.3 \times 10^{-3}(\Lambda / \mathrm{TeV})$ \\
$S_{\psi K_{s}}$ & $\sqrt{\left|\operatorname{Im}\left(c_{b d}^{2}+c_{d b}^{* 2}-6.3 c_{b d} c_{d b}^{*}\right)\right|}<1.8 \times 10^{-3}(\Lambda / \mathrm{TeV})$ \\
$\Delta m_{B_{s}}$ & $\sqrt{\left|\operatorname{Abs}\left(c_{b s}^{2}+c_{s b}^{* 2}-6.1 c_{b s} c_{s b}^{*}\right)\right|}<1.4 \times 10^{-2}(\Lambda / \mathrm{TeV})$ \\
\hline
\end{tabular}

Table 3. Bounds on off-diagonal elements of the coefficients $c_{\psi}$, defined in eq. (2.5), computed in the quark mass eigenbasis.

first contact contributions to $\digamma$ pair production appear. The SM field content is such that no dimension 5 operators exist, with the exception of the lepton number breaking Weinberg operator $(L H)^{2} / \Lambda_{L}$, which we will assume to be associated with a much larger scale $\Lambda_{L} \gg \Lambda$ and can be ignored for our present purposes. Under this assumption, there are no dimension-6 operators linear in $\digamma$. This means that the single $\digamma$ production computed from eqs. (2.5), (2.6) receives corrections only at $\mathcal{O}\left(M_{\digamma}^{2} / \Lambda^{2}\right)$ and not $\mathcal{O}\left(M_{\digamma} / \Lambda\right)$. Moreover, structures of the form $\digamma \partial_{\mu} \digamma J_{\mathrm{SM}}^{\mu}$ are proportional to $\digamma^{2} \partial_{\mu} J_{\mathrm{SM}}^{\mu}$, up to a total derivative, and can be eliminated using arguments analogous to those employed for eq. (2.5). We thus find that the most general dimension- 6 effective Lagrangian is

$$
\begin{aligned}
\mathscr{L}_{6}=\frac{\digamma^{2}}{\Lambda^{2}}[ & c_{g g}^{(6)} \frac{g_{3}^{2}}{2} G_{\mu \nu}^{a} G^{a \mu \nu}+c_{W W}^{(6)} \frac{g_{2}^{2}}{2} W_{\mu \nu}^{a} W^{a \mu \nu}+c_{B B}^{(6)} \frac{g_{1}^{2}}{2} B_{\mu \nu} B^{\mu \nu}+c_{\psi}^{(6)}\left(H \bar{\psi}_{L} \psi_{R}+\text { h.c. }\right) \\
& \left.+c_{H}^{(6)}\left|D_{\mu} H\right|^{2}-c_{H}^{(6) \prime}\left(|H|^{4}-v^{4}\right)\right]+\frac{c_{H 2}^{(6)}}{\Lambda^{2}} \frac{\left(\partial_{\mu} \digamma\right)^{2}}{2}\left(|H|^{2}-v^{2}\right)+\mathcal{O}\left(\digamma^{4}\right),
\end{aligned}
$$

where we ignore terms with at least $\digamma^{4}$ that do not have any phenomenological impact in our analysis. With the exception of the last term $c_{H 2}^{(6)}$, the terms in eq. (2.9) share the structure of the dimension-5 Lagrangian. Note that in this case the CP-even and CP-odd states have the same interactions, since $\digamma$ appears quadratically while $\mathrm{CP}$ violation could generate interactions of the form $\digamma^{2} V_{\mu \nu} \tilde{V}^{\mu \nu}\left(V=B, G^{a}, W^{a}\right)$ and a complex phase for $c_{\psi}^{(6)}$.

Whether or not eqs. (2.5) and (2.9) provide an adequate description of the processes under study, and whether higher-order terms in the effective Lagrangian can potentially play a role in the study of specific processes, depends on a number of assumptions about the underlying dynamics. The validity of the EFT cannot be determined entirely from a bottom-up perspective. We will comment on this issues in the appropriate sections below.

\section{$3 \digamma$ decays}

The effective Lagrangian expanded in the unitary gauge can be found in appendix A. 

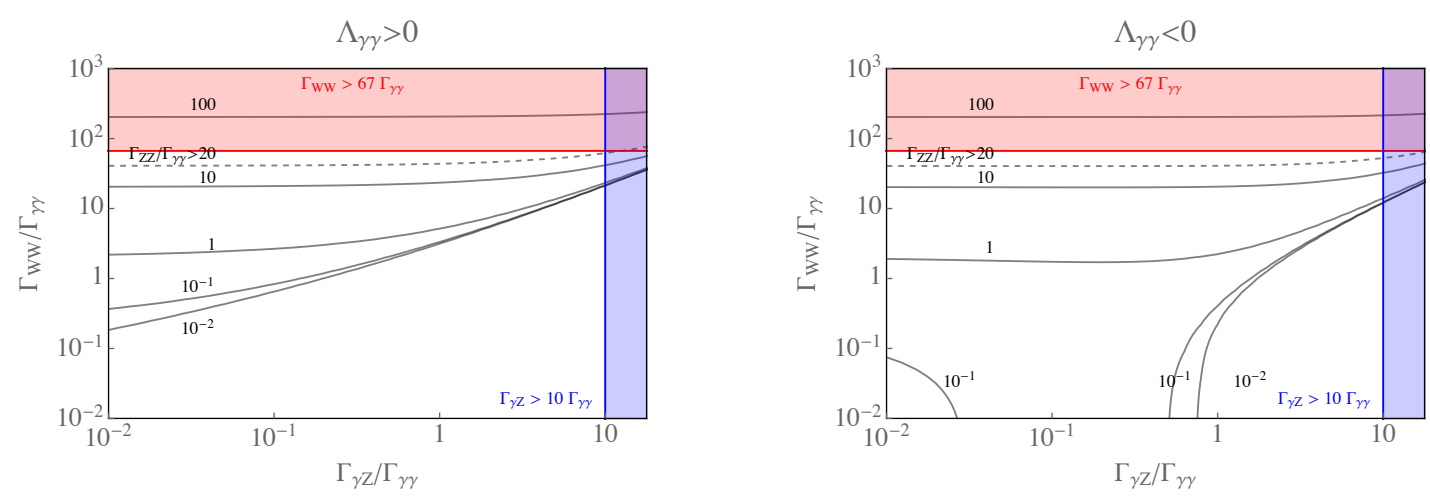

Figure 3. Predictions for $\Gamma_{Z Z} / \Gamma_{\gamma \gamma}$ as a function of $\Gamma_{\gamma Z} / \Gamma_{\gamma \gamma}$ and $\Gamma_{W W} / \Gamma_{\gamma \gamma}$ in the general effective field theory up to dimension 7 operators for a CP-even scalar. Note that the prediction does not depend on assumptions about the mixing with the Higgs boson. Two sets of predictions (left and right) are possible due to a sign ambiguity relating couplings to widths. The shaded regions and the region above the dashed line are excluded. If $\Gamma_{\gamma Z} / \Gamma_{\gamma \gamma}$ and $\Gamma_{W W} / \Gamma_{\gamma \gamma}$ were measured in the future, the prediction for $\Gamma_{Z Z} / \Gamma_{\gamma \gamma}$ could be used to unambiguously test the effective theory description.

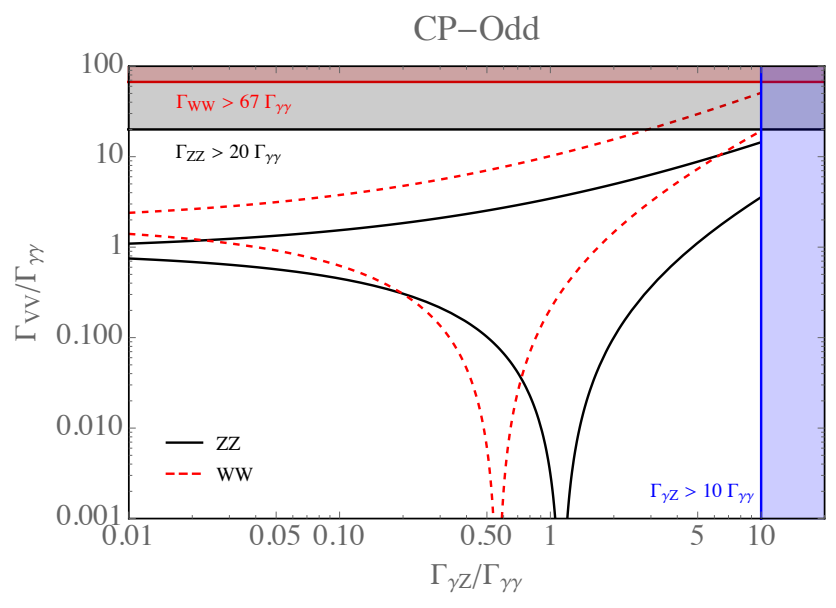

Figure 4. Predictions for $\Gamma_{Z Z} / \Gamma_{\gamma \gamma}$ and $\Gamma_{W W} / \Gamma_{\gamma \gamma}$ as a function of $\Gamma_{\gamma Z} / \Gamma_{\gamma \gamma}$ in the general effective field theory up to dimension 7 operators for a CP-odd scalar. In each case the two solutions correspond to a sign ambiguity relating couplings to widths. The shaded regions are excluded. If $\Gamma_{\gamma Z} / \Gamma_{\gamma \gamma}$ were measured in the future, these predictions for $\Gamma_{Z Z} / \Gamma_{\gamma \gamma}$ and $\Gamma_{W W} / \Gamma_{\gamma \gamma}$ could be used to test the effective theory description.

\subsection{Two-body $\digamma$ decays}

If $\digamma$ is CP-even, it can mix with the Higgs boson $h$. The mixing angle is given in eq. (B.3) of appendix B (see also [118]), and the mass eigenvalues in eq. (B.4). Equations (B.6) provide the $\digamma$ two-body widths $\Gamma_{X} \equiv \Gamma(\digamma \rightarrow X)$ taking into account the full dependence on $M_{h, t, W, Z}$. We ignore higher order operators that give corrections suppressed by $M_{\digamma}^{2} / \Lambda^{2}$. The mixing angle $\theta$ is experimentally constrained to be small, given that after mixing with 
$h, \digamma$ acquires the decay widths of a Higgs boson $h^{*}$ with mass $M_{\digamma}$ :

$$
\Gamma(\digamma \rightarrow X)=\Gamma\left(h^{*} \rightarrow X\right) \sin ^{2} \theta+\cdots, \quad \text { e.g. } \quad \Gamma\left(h^{*} \rightarrow Z Z\right) \stackrel{M_{h} \lll M_{\digamma}}{\simeq} \frac{M_{\digamma}^{3}}{32 \pi v^{2}}
$$

where $v=246 \mathrm{GeV}$ is the Higgs vacuum expectation value. Imposing the experimental bound $\Gamma(\digamma \rightarrow Z Z) \lesssim 20 \Gamma(\digamma \rightarrow \gamma \gamma)$ we obtain

$$
|\sin \theta| \lesssim 0.015 \sqrt{\frac{\Gamma(\digamma \rightarrow \gamma \gamma)}{10^{-6} M_{\digamma}}} \quad \text { (experimental bound on the } \digamma / h \text { mixing angle). }
$$

Using the complete expressions for the widths of appendix B we see that in the CP-even case the four decay widths $\Gamma_{\gamma \gamma}, \Gamma_{\gamma Z}, \Gamma_{Z Z}, \Gamma_{W^{+} W^{-}}$, are controlled by only three parameter combinations involving $c_{B B}, c_{W W}$, and $v c_{H} \cos \theta+2 \Lambda \sin \theta$. This means that once the rates relative to diphoton production $\Gamma_{\gamma Z} / \Gamma_{\gamma \gamma}$ and $\Gamma_{W^{+} W^{-}} / \Gamma_{\gamma \gamma}$ have been measured, the ratio $\Gamma_{Z Z} / \Gamma_{\gamma \gamma}$ is predicted, up to a sign ambiguity in the relation between the operator coefficients and the diphoton width. This means that, without making any assumptions on the size of the mixing with the Higgs and up to operators of dimension 7, we obtain one prediction that can be tested in future measurements. This is illustrated in figure 3 (see also [22]).

The $\digamma$ decays into EW vectors for a CP-odd scalar are described by only two parameters. Thus, once the relative rate $\Gamma_{\gamma Z} / \Gamma_{\gamma \gamma}$ is measured, both $\Gamma_{W^{+} W^{-}} / \Gamma_{\gamma \gamma}$ and $\Gamma_{Z Z} / \Gamma_{\gamma \gamma}$ are predicted, again up to a sign ambiguity. These two predictions can be tested, allowing for the determination of the $\digamma$ properties in a very model independent manner [22, 49]. This is illustrated in figure 4 .

Finally, we provide compact expressions for the widths by expanding the full expressions of eq. (B.6) for $\theta \ll 1$ and $M_{h, W, Z} \ll M_{\digamma}$ (correct up to $\sim 10 \%$ approximation): the widths reduce to the expressions of [7]:

$$
\begin{aligned}
\Gamma(\digamma \rightarrow \gamma \gamma) & =\frac{\pi \alpha^{2} M_{\digamma}^{3}}{\Lambda^{2}}\left(c_{\gamma \gamma}^{2}+\tilde{c}_{\gamma \gamma}^{2}\right), \\
\Gamma(\digamma \rightarrow g g) & =\frac{8 \pi \alpha_{3}^{2} M_{\digamma}^{3}}{\Lambda^{2}}\left(c_{g g}^{2}+\tilde{c}_{g g}^{2}\right), \\
\Gamma(\digamma \rightarrow \psi \bar{\psi}) & =\frac{N_{\psi} M_{\digamma} v^{2}}{16 \pi \Lambda^{2}}\left(c_{\psi \digamma}^{2}+\tilde{c}_{\psi \digamma}^{2}\right), \\
\Gamma(\digamma \rightarrow h h) & =\frac{M_{\digamma}^{3}}{128 \pi \Lambda^{2}} \hat{c}_{H}^{2}, \\
\Gamma(\digamma \rightarrow Z Z) & =\frac{\pi \alpha^{2} M_{\digamma}^{3}}{\Lambda^{2} s_{\mathrm{W}}^{4} c_{\mathrm{W}}^{4}}\left(c_{Z Z}^{2}+\tilde{c}_{Z Z}^{2}\right)+\frac{M_{\digamma}^{3}}{128 \pi \Lambda^{2}} \hat{c}_{H}^{2}, \\
\Gamma\left(\digamma \rightarrow W^{+} W^{-}\right) & =\frac{2 \pi \alpha^{2} M_{\digamma}^{3}}{\Lambda^{2} s_{\mathrm{W}}^{4}}\left(c_{W W}^{2}+\tilde{c}_{W W}^{2}\right)+\frac{M_{\digamma}^{3}}{64 \pi \Lambda^{2}} \hat{c}_{H}^{2}, \\
\Gamma(\digamma \rightarrow \gamma Z) & =\frac{2 \pi \alpha^{2} M_{\digamma}^{3}}{s_{\mathrm{W}}^{2} c_{\mathrm{W}}^{2} \Lambda^{2}}\left(c_{\gamma Z}^{2}+\tilde{c}_{\gamma Z}^{2}\right) .
\end{aligned}
$$

Here $s_{\mathrm{W}}$ and $c_{\mathrm{W}}$ are sine and cosine of the weak mixing angle and $N_{\psi}$ is the $\psi$ multiplicity (e.g. $N_{\psi}=3$ for an $\mathrm{SU}(2)_{L}$ singlet quark). We have defined

$$
c_{\gamma \gamma}=c_{B B}+c_{W W}, \quad c_{\gamma Z}=s_{\mathrm{W}}^{2} c_{B B}-c_{\mathrm{W}}^{2} c_{W W},
$$



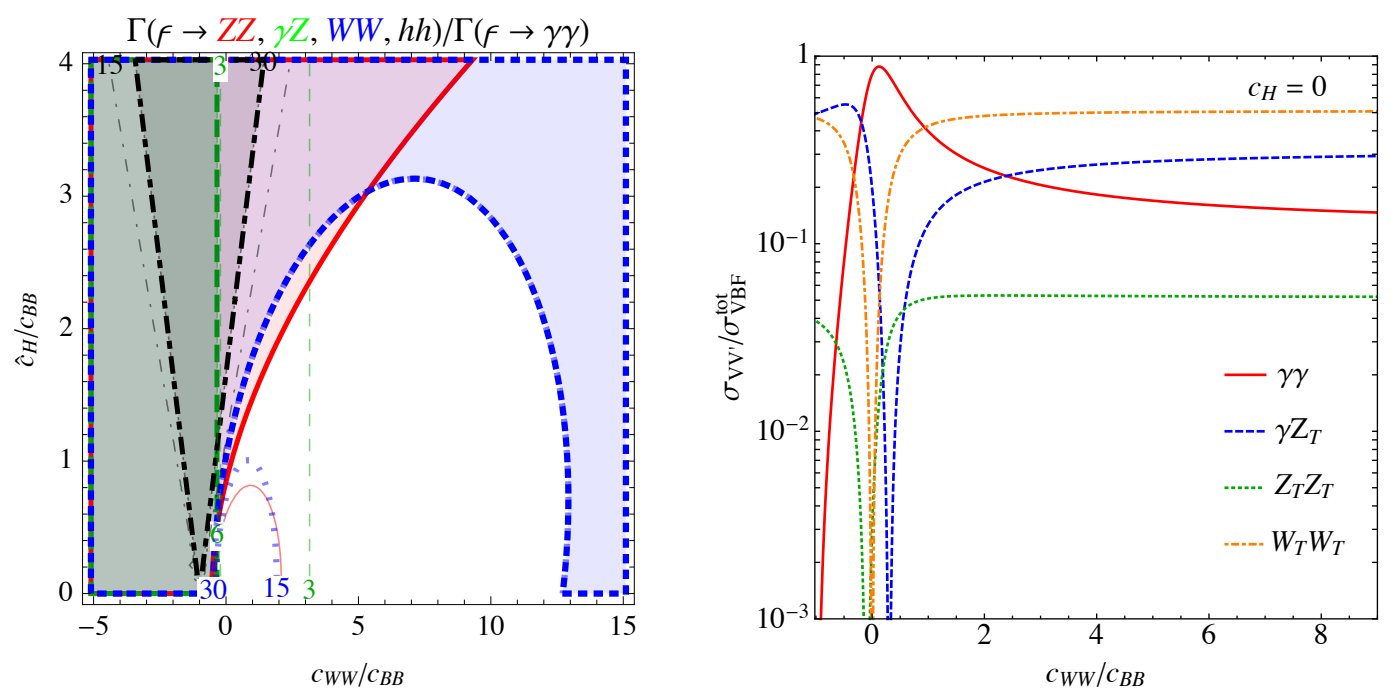

Figure 5. Left: iso-contours of $\Gamma(\digamma \rightarrow X) / \Gamma(\digamma \rightarrow \gamma \gamma)$ for $X=Z Z$ (red continuous curves), $X=\gamma Z$ (green dashed), $W W$ (blue dashed), $h h$ (black dot-dashed) as a function of $\left(c_{W W}, \hat{c}_{H}\right) / c_{B B}$. Shaded regions are excluded. Right: ratio of the production cross section by Vector Boson Fusion (VBF) in the channels $V V^{\prime}=\gamma \gamma, \gamma Z_{T}, Z_{T} Z_{T}, W_{T} W_{T}$ respectively, divided by the total VBF production cross section as a function of the ratio $c_{W W} / c_{B B}$ for $c_{H}=0$.

$$
c_{Z Z}=s_{\mathrm{W}}^{4} c_{B B}+c_{\mathrm{W}}^{4} c_{W W}, \quad \hat{c}_{H}=c_{H}+2 \kappa_{\digamma H} \Lambda / M_{\digamma} .
$$

In the $M_{h, W, Z} \ll M_{\digamma}$ limit, $\kappa_{\digamma H} m_{\digamma} \digamma|H|^{2}$ and $c_{H} \digamma|D H|^{2} / \Lambda$ are the only two operators that contribute to the $\digamma$ decays into Higgs or longitudinal vector bosons, and appear only in the combination $\hat{c}_{H}$. This is because, after the field redefinitions discussed below eq. (2.5), combinations of $c_{H}$ and $\kappa_{\digamma H}$ orthogonal to $\hat{c}_{H}$ can be eliminated in favour of other operators in eqs. (2.3), (2.5) and a combination of $\digamma^{5}$ and $\digamma^{3}|H|^{2}$, which do not contribute to 2-body $\digamma$ decays. Keeping instead terms suppressed by $M_{h, W, Z} / M_{\digamma}$, more operators contribute to the decay widths.

In the left panel of figure 5 we show the allowed values of $\left(c_{W W}, \hat{c}_{H}\right) / c_{B B}$ (white region) together with the various bounds. In the right panel of figure 5 we show the relative contributions of the $\gamma \gamma, \gamma Z_{T}, Z_{T} Z_{T}$ and $W_{T} W_{T}$ channels to VBF production cross section as a function $c_{W W} / c_{B B}$ : in the allowed range photon fusion is the dominant VBF production mechanism only in the neighbourhood of $c_{W W} \sim 0$, while the other channels become relevant, or even dominant for $\left|c_{W W}\right| \sim\left|c_{B B}\right|$. This shows that in the effective theory describing the interactions of a scalar singlet in an $\mathrm{SU}(2)_{L} \otimes \mathrm{U}(1)_{Y}$ invariant way, it is generally not possible to give a meaning to the photon-fusion production mechanism without considering also the other relevant VBF channels, unless $\left|c_{W W}\right| \ll\left|c_{B B}\right|$ (see also related discussion in $[22,96])$. 


\section{$3.2 \quad$ Three-body $\digamma$ decays}

$\digamma$ decay modes into more than two particles carry information about $\digamma$ couplings. For example, they allow us to access vector polarisations, and to deduce in this way the structure of $\digamma$ interactions with gauge fields. However these processes, occurring at higher-order, have relatively small branching ratios. We focus on two classes of special enhanced processes.

First, the $\digamma H \bar{\psi} \psi$ couplings lead to a two-body $\digamma \rightarrow \bar{\psi} \psi$ width suppressed by $v / M_{\digamma}$, while the three-body $\digamma \rightarrow H \bar{\psi} \psi$ width is unsuppressed. In the limit $v \ll M_{\digamma}$ we find

$$
\Gamma(\digamma \rightarrow \eta \bar{\psi} \psi)=\frac{N_{\psi} M_{\digamma}^{3} c_{\psi}^{2}}{1536 \pi^{3} \Lambda^{2}} \quad \text { i.e. } \quad \frac{\Gamma(\digamma \rightarrow \eta \bar{\psi} \psi)}{\Gamma(\digamma \rightarrow \bar{\psi} \psi)}=\frac{M_{\digamma}^{2}}{96 \pi^{2} v^{2}} \approx 0.98 \%
$$

where $\eta$ is any of the 4 components of the Higgs doublet $H$, namely the Higgs boson $h$ and the longitudinal polarisations of $Z$ and $W^{ \pm}$. Taking into account their masses and assuming that the fermion $\psi$ is a quark with negligible mass we find

$$
\begin{aligned}
& \frac{\Gamma(\digamma \rightarrow h \bar{u} u)}{\Gamma(\digamma \rightarrow \bar{u} u)}=\frac{\Gamma(\digamma \rightarrow h \bar{d} d)}{\Gamma(\digamma \rightarrow \bar{d} d)}=0.62 \%, \\
& \frac{\Gamma(\digamma \rightarrow Z \bar{u} u)}{\Gamma(\digamma \rightarrow \bar{u} u)}=\frac{\Gamma(\digamma \rightarrow Z \bar{d} d)}{\Gamma(\digamma \rightarrow \bar{d} d)}=0.57 \%, \\
& \frac{\Gamma\left(\digamma \rightarrow W^{+} \bar{u} d\right)}{\Gamma(\digamma \rightarrow \bar{q} q)}=\frac{\Gamma\left(\digamma \rightarrow W^{-} \bar{d} u\right)}{\Gamma(\digamma \rightarrow \bar{q} q)}=0.89 \%
\end{aligned}
$$

If $\digamma$ is produced from $q \bar{q}$ partonic scattering, one expects a sizeable three body decay width as well as associated processes discussed in section 5. Present data could already provide significant bounds, if the relevant searches are performed.

The second enhanced higher-order decay rate arises because collinear and/or soft emission of particles with mass $m$ is enhanced by infra-red logarithms $\sim \ln ^{n} M_{\digamma} / m$, where $n=$ 1,2 when a vectors splits into two vectors, and $n=1$ when it splits into fermions or scalars. At leading order in $\ln \left(M_{\digamma} / m\right)$, such phenomenon can be approximated as radiation.

The QCD effects is hidden into hadronisation. Considering for example the $\digamma G G$ or $\digamma G \tilde{G}$ couplings, we find the total rates

$$
\frac{\sigma(p p \rightarrow \digamma \rightarrow g g g)}{\sigma(p p \rightarrow \digamma \rightarrow g g)}=11 \%
$$

having imposed the cuts on jets described in the caption of table 1. After averaging on the gluon polarizations, the $\digamma G G$ or $\digamma G \tilde{G}$ couplings produce the same $\digamma \rightarrow g g g$ distributions.

The most interesting such effect concerns off-shell photons $\gamma^{*}$ (see also [102]), while for massive electro-weak bosons the contribution to 4 -fermion final states is anyway dominated by the on-shell $V V$ production (with $\ln \left(M_{\digamma} / M_{W}\right) \approx 2.2$ off-shell effects account for approximately $20 \%$ of the on-shell production [124]).

From an off-shell photon, we find

$$
\sum_{\wp} \Gamma\left(\digamma \rightarrow \gamma \wp^{-} \wp^{+}\right) \approx 22 \% \times \Gamma(\digamma \rightarrow \gamma \gamma)
$$




\begin{tabular}{|l|cccccc|cc|}
\hline decay & $I_{1}$ & $I_{2}$ & $I_{3}$ & $I_{4}$ & $I_{5}$ & $I_{6}$ & $\Gamma_{4 \ell} / \Gamma_{\gamma \gamma}$ & $\kappa_{1}$ \\
\hline eеeе & 84.3 & 84.4 & 169 & 137. & 137. & 0.0556 & $3.63 \times 10^{-4} \mp 0.235$ \\
$\mu \mu \mu \mu$ & 29.0 & 29.1 & 58.1 & 52.7 & 52.8 & 0.0556 & $1.36 \times 10^{-4} \mp 0.216$ \\
$\tau \tau \tau \tau$ & 9.45 & 9.51 & 19.0 & 19.6 & 19.6 & 0.0555 & $0.49 \times 10^{-4} \mp 0.195$ \\
$e е \mu \mu$ & 45.4 & 45.5 & 90.9 & 78.8 & 78.8 & 0.0556 & $4.12 \times 10^{-4} \mp 0.224$ \\
$e е \tau \tau$ & 24.5 & 24.5 & 49.0 & 50.9 & 51.0 & 0.0555 & $2.56 \times 10^{-4} \mp 0.194$ \\
$\mu \mu \tau \tau$ & 16.6 & 16.6 & 33.2 & 32.2 & 32.2 & 0.0555 & $1.64 \times 10^{-4} \mp 0.205$ \\
\hline
\end{tabular}

Table 4. Coefficients that define the $\digamma \rightarrow \ell^{+} \ell^{-} \ell^{\prime} \ell^{\prime-}$ distributions. In the last column, the negative (positive) sign of $\kappa_{1}$ corresponds to the CP-even(odd) case.

where $\wp$ denote final-state particle species and the sum is dominated by $\wp=W(5 \%$, thanks to double IR logarithms), $\wp=u(4 \%)$ and $\wp=e(4 \%)$. Splitting into electrons and muons is particularly important, given their small mass and given that collider experiments can precisely measure their energy and direction.

\subsection{Four-body $\digamma$ decays}

Four-body $\digamma$ decays are interesting because they allow to reconstruct the CP-parity of $\digamma$.

The largest of these decays is into gluons: we find $\sigma(p p \rightarrow \digamma \rightarrow g g g g) \approx 0.3 \% \times \sigma(p p \rightarrow$ $\digamma \rightarrow g g)$ after imposing the cuts on jets described in the caption of table 1 . The $\digamma \rightarrow$ $g^{+} g^{+} g^{-} g^{-}$amplitude (where \pm denotes the gluon helicity) depends on whether $\digamma$ is scalar or pseudo-scalar [125-127]. However, for kinematical reasons, $p p \rightarrow \digamma j j$ scatterings (section 4.1) allow us to discriminate the CP parity much better than $\digamma \rightarrow j j j j$ decays [128].

The kinematical distributions of $p p \rightarrow \digamma \rightarrow \gamma^{*} \gamma^{*} \rightarrow \ell^{+} \ell^{-} \ell^{\prime+} \ell^{\prime-}$ decay allow us to measure whether $\digamma$ is scalar or pseudo-scalar, in analogy with pion $\pi^{0}$ physics $[129,130]$. Note that these techniques find little prospects of realisation in the context of Higgs physics, due to the large di-photon background; at $750 \mathrm{GeV}$, the situation is more favourable. In our case, the rate of $\digamma$ into 4 leptons is

$$
\frac{\Gamma_{4 \ell}}{\Gamma_{\gamma \gamma}}=\frac{2 \alpha^{2}}{3 \pi^{2}} R, \quad R=\frac{S}{c_{\gamma \gamma}^{2}+\tilde{c}_{\gamma \gamma}^{2}}\left[\left(\frac{I_{1}}{2}+I_{4}\right) c_{\gamma \gamma}^{2}+\left(\frac{I_{2}}{2}+I_{5}+I_{6}\right) \tilde{c}_{\gamma \gamma}^{2}\right]
$$

where the numerical factors $I_{i}$ are reported in table 4 , and $S$ is a symmetry factor equal to $1 / 4$ when identical leptons are present $\left(\ell=\ell^{\prime}\right)$, and 1 otherwise; in the former case, the $4 \ell$ rate grows as $\ln ^{2} M_{\digamma} / m_{\ell}$.

The total rate is independent of whether $\digamma$ is a scalar or a pseudoscalar (up to terms suppressed by $m_{\ell} / M_{\digamma}$ ) and one relevant distribution to access this information follows defining $\phi$ as the relative angle between the planes of the two $\ell^{+} \ell^{-}$pairs in the centre-ofmass frame (such that for $\phi=0$ the two pairs lie in a common plane with the same-sign leptons adjacent to each other). Then, one has

$\frac{2 \pi}{\Gamma_{4 \ell}} \frac{d \Gamma_{4 \ell}}{d \phi}=1+\kappa_{1} \cos 2 \phi+\kappa_{2} \sin 2 \phi \quad$ with $\quad \kappa_{1}=S \frac{I_{2} \tilde{c}_{\gamma}^{2}-I_{1} c_{\gamma}^{2}}{2 R\left(c_{\gamma}^{2}+\tilde{c}_{\gamma}^{2}\right)}, \quad \kappa_{2}=\frac{S I_{3}}{2 R} \frac{c_{\gamma} \tilde{c}_{\gamma}}{c_{\gamma}^{2}+\tilde{c}_{\gamma}^{2}} \cos \delta$, 
and the sign of $\kappa_{1}$ discriminates the scalar case $\left(\kappa_{1} \approx-0.2\right)$ from the pseudo-scalar case $\left(\kappa_{1} \approx+0.2\right)$; their precise values are reported in table 4 . The $\kappa_{2}$ term violates $\mathrm{CP}$ and is present only when both couplings are present, and $\delta$ is the phase difference between the scalar and pseudo-scalar coupling. More details and distributions can be found in [129].

\section{$4 \quad p p \rightarrow \digamma j, \digamma j j:$ associated production with jets}

In the previous section we have discussed examples where more complicated processes involving $\digamma$, albeit having small rates, contain important information about the nature of $\digamma$. Associated production with additional hard jets falls in the same category. The relevant cross sections $\sigma_{\digamma j, \digamma j j}$ for producing $\digamma$ together with one or two jets (including $b$ jets) at the $13 \mathrm{TeV}$ LHC are

$$
\begin{aligned}
\sigma(p p \rightarrow \digamma j) & =\frac{\mathrm{TeV}^{2}}{\Lambda^{2}}\left[164 c_{g g}^{2}+0.51 c_{u}^{2}+0.30 c_{d}^{2}+0.03 c_{s}^{2}+0.022 c_{c}^{2}+0.012 c_{b}^{2}\right] \mathrm{pb} \\
\sigma(p p \rightarrow \digamma b) & =\frac{\mathrm{TeV}^{2}}{\Lambda^{2}}\left[1.95 c_{g g}^{2}+0.008 c_{b}^{2}\right] \mathrm{pb} \\
\sigma(p p \rightarrow \digamma j j) & =\frac{\mathrm{TeV}^{2}}{\Lambda^{2}}\left[29 c_{g g}^{2}+0.088 c_{u}^{2}+0.05 c_{d}^{2}+10^{-3}\left(4.2 c_{s}^{2}+3 c_{c}^{2}+1.8 c_{b}^{2}\right)\right] \mathrm{pb} \\
\sigma(p p \rightarrow \digamma j b) & =\frac{\mathrm{TeV}^{2}}{\Lambda^{2}}\left[0.59 c_{g g}^{2}+10^{-3}\left(26 c_{u}^{2}+15 c_{d}^{2}+0.84 c_{s}^{2}+0.52 c_{c}^{2}+1.6 c_{b}^{2}\right)\right] \mathrm{pb} \\
\sigma(p p \rightarrow \digamma b b) & =\frac{\mathrm{TeV}^{2}}{\Lambda^{2}}\left[0.15 c_{g g}^{2}+10^{-3}\left(26 c_{u}^{2}+15 c_{d}^{2}+0.8 c_{s}^{2}+0.5 c_{c}^{2}+0.4 c_{b}^{2}\right)\right] \mathrm{pb} .
\end{aligned}
$$

We ignore interferences in $\digamma j j, \digamma j b, \digamma b b$ cross sections. The operators coupling $\digamma$ to two EW vector bosons, both longitudinal and transverse, have not been considered here, because they contribute to the VBF topology, which already contains two forward jets. Here we implemented the following cuts to single out hard jets: $\eta<5$ on all rapidities, $p_{T}>150 \mathrm{GeV}$ on all transverse momenta, and angular difference $\Delta R>0.4$ for all jet pairs. These results are summarised in the first five lines of table 1 , shown in units of the leading results $\sigma_{\digamma}$ from eq. (2.7).

A measure of $\sigma_{\digamma j} / \sigma_{\digamma}$, which in some cases is expected to be relatively large (see table 1), can discriminate between different initial states: the $\digamma G G$ operator leads to more initial-state jet radiation than the $\digamma q \bar{q}$ operators. This was discussed in ref. [41] which proposed the average $p_{T}$ of $\digamma$ as a good discriminator. In this analysis, and throughout the whole article, we are implicitly assuming that higher order terms in the EFT expansion are under control also for processes that can potentially probe the high-energy region, such as $\digamma j$ or $\digamma j j$ associated production. We shall discuss this in more detail in section 5.2, but here we mention that these effects are associated with operators of dimension-7 or higher that can be in the form of direct contact contributions, such as $\digamma G_{\mu \nu}^{a} G_{\rho}^{b \nu} G^{c \rho \mu} \epsilon^{a b c}$ (in a microscopic model with loops of heavy coloured states $\mathcal{Q}$, this corresponds to emission of the jet directly from $\mathcal{Q}$ ), or higher derivative terms; in both cases they are suppressed by two powers of the large scale $\Lambda$.

In order to assess the validity of the EFT for the associated production with jets, we compared the corresponding numbers in table 1 with the ones obtained in an explicit model 
featuring a new coloured fermion with masses in the range $500 \mathrm{GeV}-10 \mathrm{TeV}$. We found that for all associated productions with jets the deviation from the EFT prediction is smaller than $\sim 35 \%$ for a fermion mass of $500 \mathrm{GeV}$ (which is hardly compatible with limits from direct searches) and smaller than $\sim 10 \%$ for a fermion mass of $1 \mathrm{TeV}$. This shows that given the rather strong constraints on the masses of new coloured particles coming from direct searches, the results of the EFT are rather robust.

\subsection{CP of $\digamma$ from $p p \rightarrow \digamma j j$}

The differential distribution of the $\digamma j$ and $\digamma b$ cross sections does not allow us to discriminate a scalar $\digamma$ from a pseudo-scalar $\digamma$. For example the gluonic and quark operators contribute as

$$
\begin{aligned}
& \frac{d \sigma}{d t}(g g \rightarrow \digamma g)=\frac{3 g_{3}^{6}}{128 \pi s^{2} \Lambda^{2}}\left(c_{g g}^{2}+\tilde{c}_{g g}^{2}\right) \frac{M_{\digamma}^{8}+s^{4}+t^{4}+u^{4}}{s t u} \\
& \frac{d \sigma}{d t}(q \bar{q} \rightarrow \digamma g)=\frac{g_{3}^{2}}{36 \pi s^{2} \Lambda^{2}}\left[\left(c_{g g}^{2}+\tilde{c}_{g g}^{2}\right) \frac{g_{3}^{4}\left(t^{2}+u^{2}\right)}{s}+\frac{v^{2}}{2}\left(c_{q}^{2}+\tilde{c}_{q}^{2}\right) \frac{M_{\digamma}^{4}+s^{2}}{t u}\right] \\
& \frac{d \sigma}{d t}(g q \rightarrow \digamma q)=\frac{-g_{3}^{2}}{96 \pi s^{2} \Lambda^{2}}\left[\left(c_{g g}^{2}+\tilde{c}_{g g}^{2}\right) \frac{g_{3}^{4}\left(s^{2}+u^{2}\right)}{t}+\frac{v^{2}}{2}\left(c_{q}^{2}+\tilde{c}_{q}^{2}\right) \frac{M_{\digamma}^{4}+t^{2}}{s u}\right]
\end{aligned}
$$

with $s+t+u=M_{\digamma}^{2}$ (see also the analogous Higgs cross sections [131]). On the other hand, production of $\digamma$ in association with two jets provides kinematic distributions that are sensitive to the $\mathrm{CP}$ nature of $\digamma$. A well known variable that is sensitive to the $\mathrm{CP}$ nature of $\digamma$ is the azimuthal angle between the two jets $\Delta \phi_{j j}[128,132,133]$. In principle other jet distributions are also sensitive to the CP nature of $\digamma$. For instance, [134] has examined a set of jet shape variables for the determination of the CP nature of a SMlike Higgs boson, which are potentially interesting for $\digamma$ as well. In the following we will examine the sensitivity to the $\mathrm{CP}$ nature of $\digamma$ of the thrust of the hard jets in the event

$$
T=\max _{n} \frac{\sum_{i \in \text { jets }}\left|n \cdot p_{i}\right|}{\sum_{i \in \text { jets }}\left|p_{i}\right|} .
$$

This variable, unlike $\Delta \phi_{j j}$, exploits both transverse and longitudinal momentum of the jets, hence carries independent information on the $\mathrm{CP}$ nature of $\digamma$ which can be in principle combined with that carried by the $\Delta \phi_{j j}$ distribution. Furthermore $\Delta \phi_{j j}$ and the thrust are expected to have different sensitivities to QCD aspects such as hadronization or soft and collinear emissions, so that it is useful to cross-check the impact of these effects. Similar considerations apply to different experimental effects.

Given the differences between the SM Higgs boson and $\digamma$, it is worth reassessing the validity of the choices that are standard for studies of the SM Higgs boson, keeping in mind that $\digamma$ is significantly heavier than the Higgs boson. Hence, all effects related to the velocity of $\digamma$ or the recoil of the two jets against the scalar are less useful. Another important difference is that for the case of the Higgs boson two contributions, one from gluon fusion and one from vector boson fusion, are normally considered and often selection cuts are imposed to reduce the former and retain the latter. For $\digamma$ this could be a meaningful choice if it will be demonstrated that the production mechanism is mainly from photon 
or electroweak boson fusion processes, so that $\digamma$ is produced in a hard process without accelerated colour charges and features such as a rapidity gap in the distribution of hadrons is expected. For the time being this situation is not favoured and it seems more likely that $\digamma$ production involves accelerated colour charges, requiring a reassessment of the strategy to isolate the signal from the background. This consideration is further reinforced by the fact that the possible decays of $\digamma$ are not known yet. Relying on the existence of the diphoton decay mode, the relevant final state would be

$$
p p \rightarrow \digamma j j \rightarrow \gamma \gamma j j
$$

which is usually not considered for the SM Higgs (however see [135] and references therein for early studies of this final state for the SM Higgs boson). On top of the above differences with respect to the SM Higgs boson, another crucial aspect is the rate of signal events, which for the $\gamma \gamma$ final state might be a fraction of $\mathrm{fb}$, once two extra jets are required. This forces a careful choice of the strategy to distinguish the two CP hypothesis.

The irreducible background from SM processes arises from $p p \rightarrow \gamma \gamma j j$ and in general appears to be not negligible compared to the expected signal rate. As a matter of fact our calculations below show that the overall signal-to-background ratio for the $\gamma \gamma j j$ final state is smaller than the one for the observed resonant $\gamma \gamma$ bump. Background and signal differential cross-section are computed at leading order with MadGraph5 without improvements beyond the fixed order at which we compute each process. The jets are defined as quarks or gluons around which no other quark or gluon is found in a region of angle $\Delta R=\left[(\Delta \phi)^{2}+(\Delta \eta)^{2}\right]^{1 / 2}=0.4$. Furthermore a quark or gluon considered as jet must lie in the geometrical and $p_{T}$ acceptance

$$
\left|\eta_{j}\right|<5, \quad p_{T}>75 \mathrm{GeV} .
$$

For photons we require

$$
p_{T, \gamma_{1}}>40 \mathrm{GeV}, \quad p_{T, \gamma_{2}}>30 \mathrm{GeV}, \quad \eta_{\gamma}<2.37, \quad 700 \mathrm{GeV}<m_{\gamma \gamma}<800 \mathrm{GeV} .
$$

With these definitions of hard jets and photons we find

$$
\frac{\sigma_{\mathrm{sig}}(j j \gamma \gamma)}{\sigma_{\mathrm{sig}}(\gamma \gamma)}=0.16, \quad \frac{\sigma_{\mathrm{bck}}(j j \gamma \gamma)}{\sigma_{\mathrm{bck}}(\gamma \gamma)}=0.30
$$

where the larger fraction of background diphoton events with jets arises in part by the collinear enhancement for obtaining photons from quark fragmentation in large invariant mass dijet events as well as multiplicity factors for jet emissions and "internal bremsstrahlung" from off-shell intermediate states of the diphoton background process. ${ }^{7}$ In principle one can devise selections to increase the signal-to-background ratio, e.g. by requiring harder isolation between jets and photon to reject the background from jet fragmentation. However, we do not find this useful in view of the limited amount of signal

\footnotetext{
${ }^{7}$ Additional backgrounds can arise from jets being misreconstructed as isolated photons. In $p p \rightarrow \digamma$ analyses, such backgrounds have been found to constitute less than $10 \%$ of the total background $[1,2]$.
} 
events that we can anticipate. At this stage the two CP hypothesis can already be distinguished as demonstrated in figure 6 by the $\Delta \phi_{j j}$ distribution. Still the distinction between the two CP hypotheses can be ameliorated by imposing selections that affect the shape of the distributions. For instance we note that in the low $\Delta \phi_{j j}$ region the distribution is heavily influenced by the isolation requirements for the jets, which are not CP-sensitive, and at large $\Delta \phi_{j j}$, where the two distributions are most different, the background is larger, and steeply varying. For this reason it is worth exploring possible further selections to make the differences between the distributions expected for the two CP hypothesis visible in a region of $\Delta \phi_{j j}$ where the background is low and possibly flat. To this end we identified $\left|\Delta \eta_{j j}\right|$ and $m_{j j}$ as possible variables on which to impose cuts. We remark that, unlike the SM Higgs analyses aimed at isolating VBF Higgs production, selections on these variables do not necessarily increase the inclusive signal-to-background ratio. Nonetheless, we find them helpful to identify the $\mathrm{CP}$ nature of $\digamma$. For instance requiring

$$
m_{j j}>500 \mathrm{GeV} \quad \text { and } \quad\left|\Delta \eta_{j j}\right|>2.5,
$$

a fraction about $20 \%$ of both signal events and background events are retained and the probability density of $\Delta \phi_{j j}$ distribution is shown in figure 6. The main effect of these selections is to eliminate the constraints on the jet $\Delta \phi_{j j}$ from jet isolation requirements, hence they can be relatively mild compared to standard VBF Higgs analysis.

In order to estimate the luminosity needed to identify the CP nature of $\digamma$ we use the expected distributions to draw sets of $N_{\mathrm{ev}}$ pseudo-events. We compute the likelihood ratio

$$
\mathcal{L}=-2 \ln \prod_{i=1 \ldots N_{\mathrm{ev}}} \frac{p d f\left(\mathrm{CP}-\text { odd }, \Delta \phi_{i}\right)}{p d f\left(\mathrm{CP}-\text { even, } \Delta \phi_{i}\right)}
$$

where $\Delta \phi_{i}$ are the $\Delta \phi$ values of each pseudo-experiment. Performing a large number of pseudo-experiments, as customary in these analyses [136], we take the likelihood ratio above as our test-statistics to distinguish the two $\mathrm{CP}$ options for $\digamma$. The distribution of the test-statistics for the baseline selection with the extra cuts in eq. (4.8) are reported in the two panels in the middle row of figure 6 for $N_{\mathrm{ev}}=100$ events and 20 events, respectively. Given the efficiency of the cuts in eq. (4.8) the two panels correspond to the same integrated luminosity $\mathcal{L} \sim 100 \mathrm{fb}^{-1} \times 6 \mathrm{fb} / \sigma_{\text {sig }}(\gamma \gamma)$. Considering the area of the tail of the CP-even distribution above the $\mathrm{CP}$-odd median, we find that the $\mathrm{CP}$-even hypothesis can be rejected with $90 \%$ C.L. and, adding the cuts in eq. (4.8), above 95\% C.L. Similar results hold for the converse exclusion. In the bottom row of figure 6 we show the distribution of the teststatistics when in the $p d f$ for each CP hypothesis we add the $p d f$ of the SM background with rate twice that of the signal, as suggested by eq. (4.7). The inclusion of background deteriorates the exclusions, which drop to $85 \%$ C.L. and 95\% C.L., respectively. Results on an observable similar to $\Delta \phi_{j j}$ have been discussed in [96], which claims similar results.

For the thrust we find similar results, which are illustrated in figure 7 and are obtained with the same procedure as for $\Delta \phi_{j j}$. With the same number of events as above we expect an exclusion at $88 \%$ C.L. for the analysis without the cuts in eq. (4.8) and above 95\% C.L. adding these cuts. Including the background in the same way as for the study of $\Delta \phi_{j j}$ we 

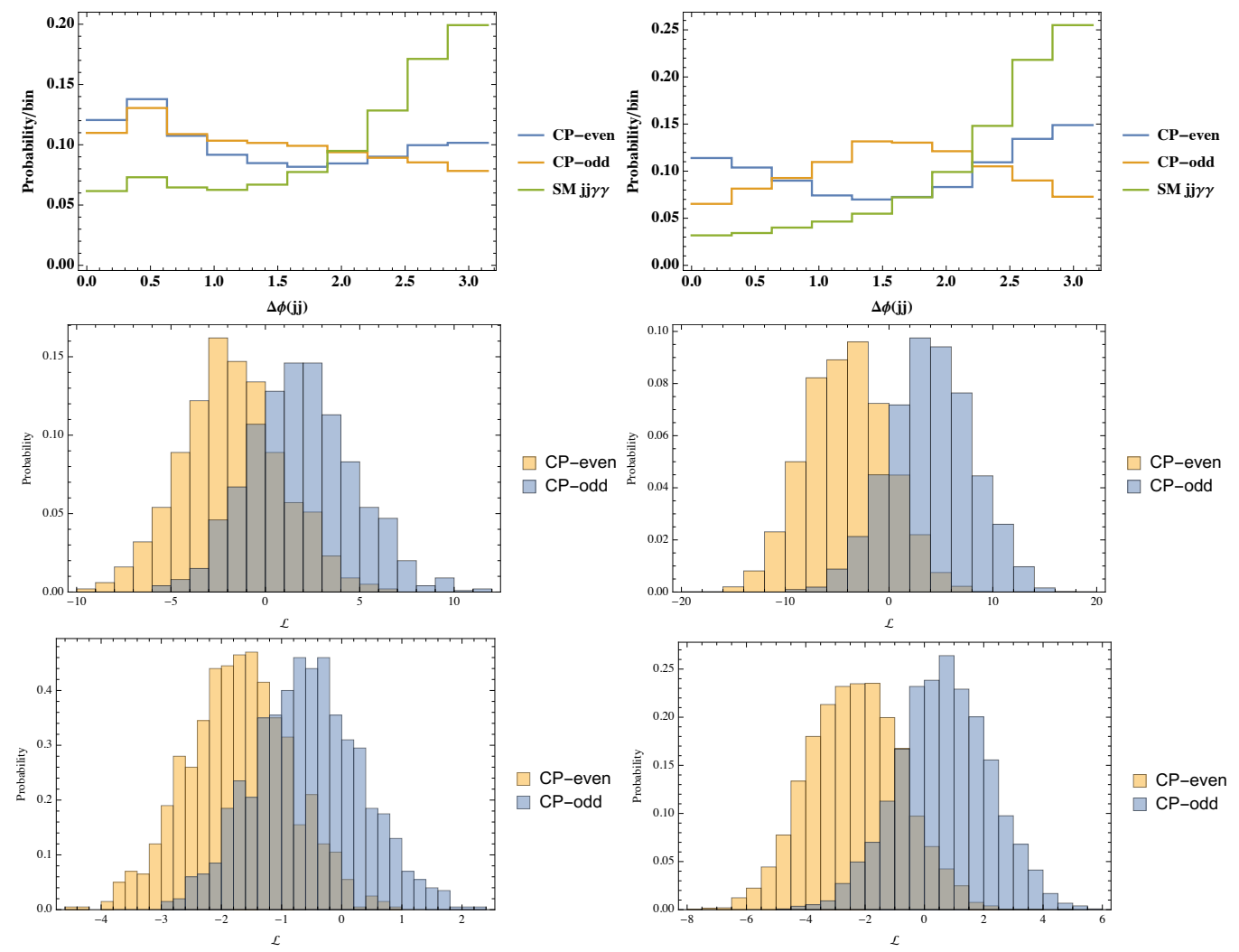

Figure 6. Upper row: normalised $\Delta \phi_{j j}$ distributions in $p p \rightarrow \gamma \gamma j j$ events for the CP-even (blue) and CP-odd (yellow) hypothesis as well as for the irreducible SM background $\gamma \gamma j j$ (green). In the left panel we impose only the minimal selection to have $\digamma \rightarrow \gamma \gamma$ and two jets, while in the right panel we impose the extra requirements in eq. (4.8) to enhance the difference between the two CP hypothesis. Middle row: distribution of the test-statistics in absence of background. Bottom row: distribution of the test statistics for a total background rate twice the signal rate, as indicated by eq. (4.7).

expect the exclusion to drop at $65 \%$ C.L. and around $75 \%$ C.L. for the two cut options, respectively.

The combination of the results from $\Delta \phi_{j j}$ and the thrust is meaningful once one takes into account their correlation. For illustration we show the doubly differential distribution in the plane $\left(T, \Delta \phi_{j j}\right)$ for the CP-even and CP-odd hypotheses as well as for the background.

If $\digamma$ couples to quarks, rather than to gluons, the difference between CP-odd and $\mathrm{CP}-$ even distributions gets suppressed by small quarks masses, and is not observable.

\section{$5 p p \rightarrow \digamma V, \digamma h:$ EW associated production}

Production of $\digamma$ in association with EW bosons provides an additional handle to distinguish different initial states and the structure of their couplings to $\digamma$. The cross sections $\sigma(p p \rightarrow$ $\digamma V) \equiv \sigma_{\digamma V}$ for producing $\digamma$ together with an SM vector (see also [59]) or with the Higgs 

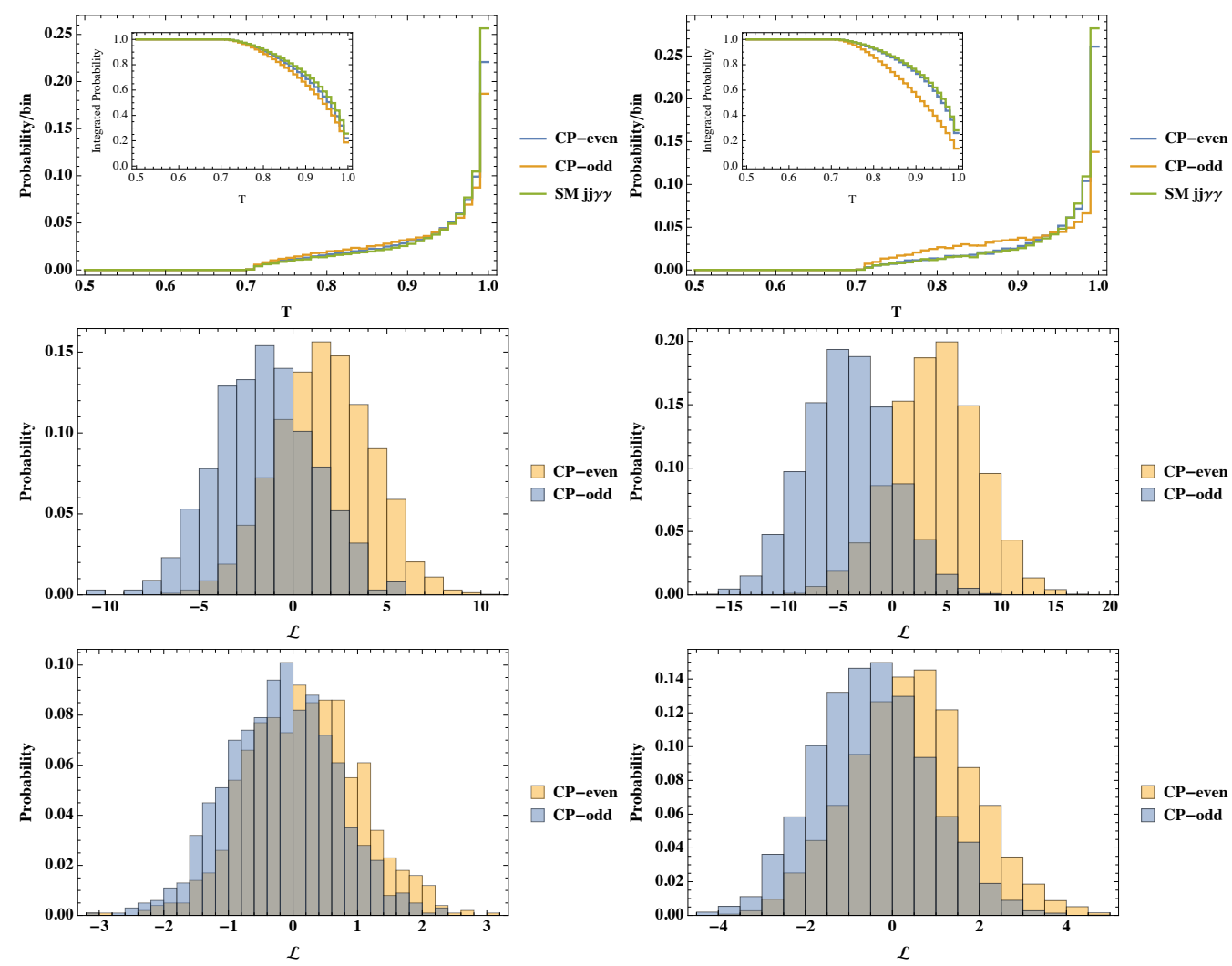

Figure 7. Upper row: normalised thrust distributions in $p p \rightarrow \gamma \gamma j j$ events for the CP-even (blue) and CP-odd (yellow) hypothesis as well as for the irreducible SM background $\gamma \gamma j j$ (green). The inset in each panel shows the cumulative distribution, which highlights the differences between the shapes of the distributions. In the left panel we impose only the minimal selection to have $\digamma \rightarrow \gamma \gamma$ and two jets, while in the right panel we impose the extra requirements in eq. (4.8) to enhance the difference between the two CP hypothesis. Middle row: distribution of the test-statistics in absence of background. Bottom row: distribution of the test statistics for a total background rate twice the signal rate, as indicated by eq. (4.7).
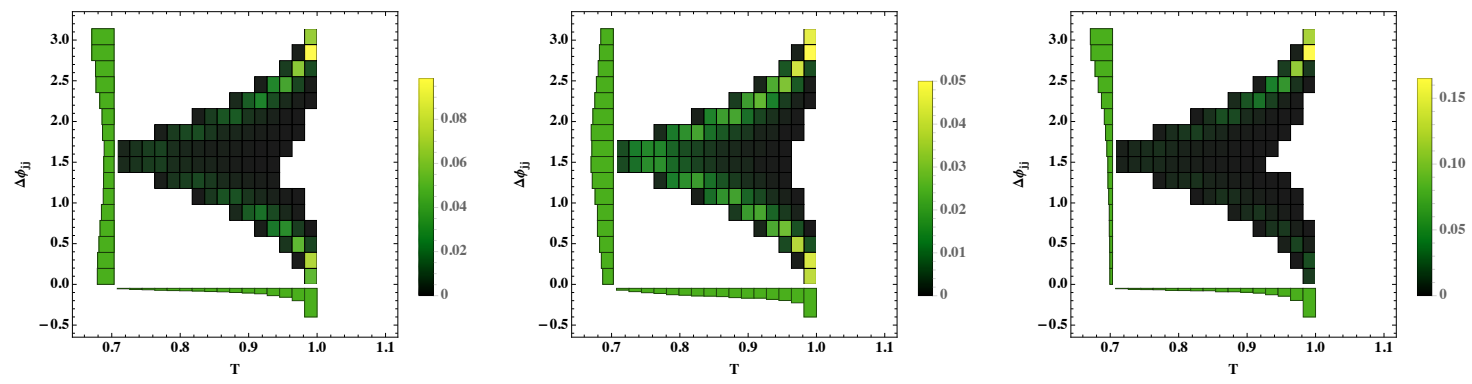

Figure 8. Double differential $\left(T, \Delta \phi_{j j}\right)$ probability distribution of CP-even (left), CP-odd (middle) and background (right) after the cuts eq. (4.8). 

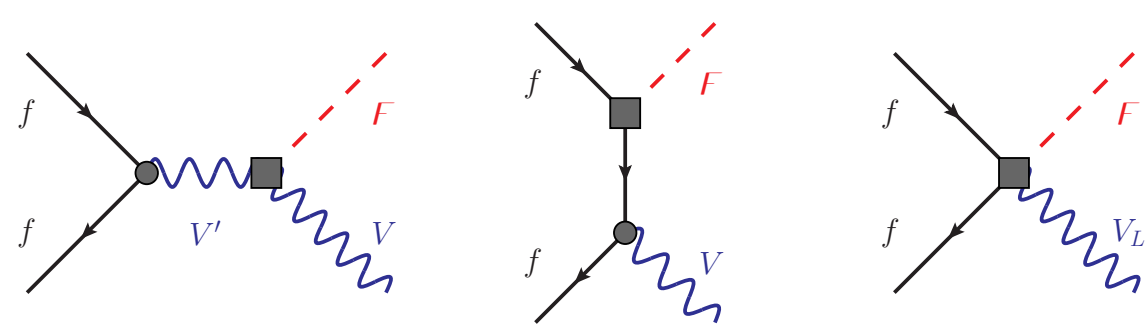

Figure 9. Diagramatic representation of partonic processes contributing to $\digamma V$ associated production due to $\digamma$ couplings to EW gauge bosons (left-hand diagram) and SM fermions (middle and right-handed diagram). The $\digamma$ interaction vertices derived from eq. (2.5) are marked with a box, while the gauge interaction vertices of SM fermions are marked with a disk.

boson, receive contributions from diagrams such as those in figure 9. At the $13 \mathrm{TeV}$ LHC, for the CP-even case, we find

$$
\begin{aligned}
\sigma(p p \rightarrow \digamma \gamma)= & \frac{\mathrm{TeV}^{2}}{\Lambda^{2}}\left[0.12 c_{u}^{2}+1.9 \times 10^{-2} c_{d}^{2}+1.6 \times 10^{-3} c_{s}^{2}+4.4 \times 10^{-3} c_{c}^{2}+\right. \\
& \left.+4.9 \times 10^{-4} c_{b}^{2}+8.5 \times 10^{-5} c_{B B}^{2}+6.6 \times 10^{-4} c_{W W}^{2}+3.2 \times 10^{-5} c_{B B} c_{W W}\right] \mathrm{pb} \\
\sigma(p p \rightarrow \digamma Z)= & \frac{\mathrm{TeV}^{2}}{\Lambda^{2}}\left[0.15 c_{u}^{2}+9.1 \times 10^{-2} c_{d}^{2}+5.5 \times 10^{-3} c_{s}^{2}+3.3 \times 10^{-3} c_{c}^{2}+\right. \\
& +1.4 \times 10^{-3} c_{b}^{2}+2.7 \times 10^{-5} c_{B B}^{2}+2.3 \times 10^{-3} c_{W W}^{2} \\
& \left.-3.2 \times 10^{-5} c_{B B} c_{W W}+1.9 \times 10^{-3} c_{g g}^{2}+8.2 \times 10^{-6} \hat{c}_{H}^{2}\right] \mathrm{pb} \\
\sigma\left(p p \rightarrow \digamma W^{+}\right)= & \frac{\mathrm{TeV}^{2}}{\Lambda^{2}}\left[0.2 c_{u}^{2}+0.19 c_{d}^{2}+1.0 \times 10^{-2} c_{s}^{2}+5.1 \times 10^{-3} c_{c}^{2}+\right. \\
& \left.+4.9 \times 10^{-6} c_{b}^{2}+4.7 \times 10^{-3} c_{W W}^{2}+1.1 \times 10^{-5} c_{H}^{2}\right] \mathrm{pb} \\
\sigma\left(p p \rightarrow \digamma W^{-}\right)= & \frac{\mathrm{TeV}^{2}}{\Lambda^{2}}\left[7.7 \times 10^{-2} c_{u}^{2}+7.8 \times 10^{-2} c_{d}^{2}+5.1 \times 10^{-3} c_{s}^{2}+7.0 \times 10^{-3} c_{c}^{2}+\right. \\
& \left.+4.2 \times 10^{-6} c_{b}^{2}+1.8 \times 10^{-3} c_{W W}^{2}+4.5 \times 10^{-6} c_{H}^{2}\right] \mathrm{pb} \\
\sigma(p p \rightarrow \digamma h)= & \frac{\mathrm{TeV}^{2}}{\Lambda^{2}}\left[0.14 c_{u}^{2}+8.5 \times 10^{-2} c_{d}^{2}+5.2 \times 10^{-3} c_{s}^{2}+3.3 \times 10^{-3} c_{c}^{2}+\right. \\
& \left.+1.4 \times 10^{-3} c_{b}^{2}+6.6 \times 10^{-4} c_{g g}^{2}+0.12 \times 10^{-6} c_{H}^{2}\right] \mathrm{pb} \\
& -\frac{\mathrm{TeV}^{\Lambda}}{\Lambda} 0.35 \cdot 10^{-6} c_{H} \kappa_{\digamma H} \mathrm{pb}+0.4 \cdot 10^{-6} \kappa_{\digamma H}^{2} \mathrm{pb}
\end{aligned}
$$

We imposed the cuts $\eta_{\gamma}<2.5$ and $p_{T, \gamma}>10 \mathrm{GeV}$ for the photon, and no cut for the massive vectors. The numerical values have been obtained using MadGraph5 and the NNPDF LO pdf set with a running factorization scale $\mu_{F}=\sqrt{M_{\digamma}^{2}+p_{T}^{2}}$. Higher order QCD corrections can be important for these processes, but are not expected to change our results by more than $\mathcal{O}(1)$ factors. For the top quark loop contribution to $g g \rightarrow h \digamma$ production we have used the automatic loop calculation available with MadGraph5. In the massless fermion limit, the helicity structure of the amplitude proportional to $c_{V V}$ differs from the $c_{\psi}$ one, due to the chiral-breaking nature of these scalar-fermion interactions. For this reason, the only interference between the different dimension-5 interactions occurs for $c_{B B}$ and $c_{W W}$ in their contributions to vertices with photons and $Z$-bosons. Eqs. (5.1) hold for the CP-even case and become slightly different in the CP-odd case, as discussed below. 


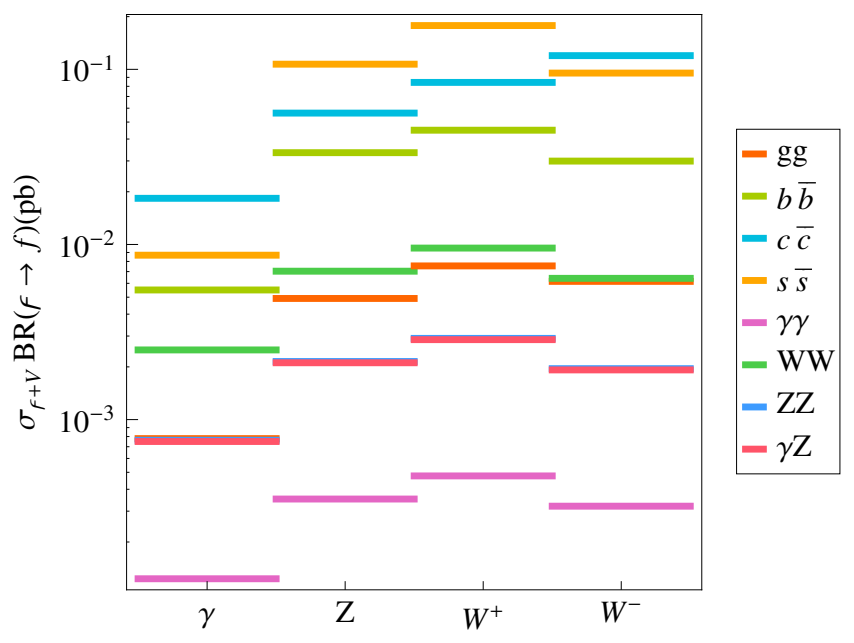

Figure 10. Maximal $\digamma V$ associate production cross-section $\sigma_{\digamma V}$ at the $13 \mathrm{TeV}$ LHC times the $\digamma \rightarrow$ $f$ branching fraction $\operatorname{BR}(\digamma \rightarrow f)$ for various possible $V=\gamma, Z, W^{+}, W^{+}$(on the horizontal axis) and $f=g g, b \bar{b}, c \bar{c}, s \bar{s}, \gamma \gamma, W^{+} W^{-}, Z Z, \gamma Z$ (different coloured bands as specified in the legend) states, subject to current experimental constraints. Values below the individual contours are possible and allowed by current data. See the main text for details.

In eq. (5.1) (and the analogous results summarised in table 1) we can identify several interesting features. In the limit where $\digamma$ is principally produced through quarks, associated EW production is always dominated by the center and right diagrams of figure 9 (contributions from the first diagram could be larger only when prompt $\digamma$ production is dominated by the $\gamma \gamma \rightarrow \digamma$ channel). Then, the ratio $\sigma_{\digamma V} / \sigma_{\digamma}$, and the production rates $p p \rightarrow V(\digamma \rightarrow \gamma \gamma)$, are independent of the total $\digamma$ width and only depend on the $q$ flavour. Processes like this one (or $\digamma \rightarrow \gamma \gamma * \rightarrow \gamma \ell^{-} \ell^{-}$discussed in section 3.2), whose amplitudes are constructed from the main amplitude $p p \rightarrow \digamma \rightarrow \gamma \gamma$ with the addition of a SM vertex, are important since their rates can be determined model-independently. For example, we obtain $\sigma_{\digamma \gamma} / \sigma_{\digamma}=\{4,16,4\} \times 10^{-3}$, for $q=\{s, c, b\}$, a prediction that could be used to single out $q q$ production channels.

Another handle for discriminating between different parton initial states is $\digamma W^{ \pm}$associated production. Assuming flavour diagonal new physics, in the case of pure $b \bar{b}$ annihilation, $\digamma W$ production is suppressed, since the contribution from initial state top quarks is negligible, while contributions from lighter initial state quarks are CKM suppressed. On the other hand, for the $s \bar{s}(c \bar{c})$ cases, the $\digamma W^{+}\left(\digamma W^{-}\right)$channel is expected to be the dominant mode because the production process can be initiated by valence quarks at the price of only Cabibbo angles.

In figure 10 we show a combination of the results of eq. (5.1) with present collider constraints, in a way that makes the expectations for EW associated production more manifest. The figure shows the maximum cross section for a given final state (different colours in the legend) produced by $\digamma$ in association with a vector (horizontal axis in 
the plot), under the following conditions: i) the total $\digamma$ decay width is $\Gamma \lesssim 45 \mathrm{GeV}$; ii) the $\digamma$ partial widths in each channel are constrained by $8 \mathrm{TeV}$ and $13 \mathrm{TeV}$ data (as specified in table 1 of [7]); iii) we maximise over different production channels, but require $\sigma_{p p \rightarrow \digamma}^{13 \mathrm{TeV}} / \sigma_{p p \rightarrow \digamma}^{8 \mathrm{TeV}} \gtrsim 4$, to ensure compatibility between $8 \mathrm{TeV}$ and $13 \mathrm{TeV}$ data.

We observe that the largest allowed rates are $\sigma_{\digamma+W, Z} \times \mathrm{BR}(\digamma \rightarrow j j) \lesssim 0.1 \mathrm{pb}$ and are saturated when $\digamma$ is predominantly produced from $s \bar{s}$ and/or $c \bar{c}$ annihilation. This signature might however be challenging to access experimentally due to the $W, Z+$ jets (and $t \bar{t}$ ) backgrounds. Recent studies in the $b b Z$ channel, searching for resonances both in the $b b$ invariant mass and in the $Z b b$ invariant mass spectrum, place bounds at the level of $0.5 \mathrm{pb}$ [138]. Finally, among the purely EW final states, $\sigma_{\digamma+W, Z} \times \mathrm{BR}\left(\digamma \rightarrow W^{+} W^{-}\right)$could still reach $\mathcal{O}(10 \mathrm{fb})$, while all photonic signatures of $\digamma V$ production are already bounded below $\mathcal{O}(\mathrm{fb})$.

Concerning EW-induced associated $\digamma$ production (the left diagram of figure 9), the largest possible rates are actually expected when prompt single $\digamma$ production is dominated by $b \bar{b}$ annihilation. Then, the photonic contribution to the first diagram in figure 9 gives $\sigma_{\digamma \gamma} \simeq 0.21-1 \mathrm{fb}$ depending on whether the width is saturated by $\Gamma_{b \bar{b}}$ or by other channels (in this latter case, a minimum $\Gamma_{b \bar{b}} \gtrsim 10 \Gamma_{\gamma \gamma}$ is still necessary to guarantee dominance of $\bar{b} b$ production over $\gamma \gamma$ production, which would be in tension with the $8 \mathrm{TeV} / 13 \mathrm{TeV}$ comparison). On the other hand, contributions of vertices involving $W, Z$ to the left diagrams in figure 9 can lead to one order of magnitude larger rates for all $\sigma_{\digamma V}$, for the simple reason that the constraints on these couplings are an order of magnitude weaker, see table 2. We close this discussion by noting that EW associated production is also one of the few model-independent $\digamma$ production process which can be probed at $e^{+} e^{-}$ colliders [18] or photon $[60,61]$ colliders.

\subsection{CP of $\digamma$ from $p p \rightarrow \digamma Z, \digamma W$}

EW associated production also allows us to test the CP nature of $\digamma$ interactions. In the case of the $\digamma Z \gamma$ coupling, measuring the $Z$ polarization in $\digamma \rightarrow Z \gamma$ decays is not enough to disentangle its $\mathrm{CP}$ nature [139]. On the other hand, in $\digamma Z$ associated production, the intermediate photon is virtual and the longitudinal polarisation of the $Z$ is accessible close to threshold and can be used to probe the CP nature of this interaction. We will consider this in the context of the CP-even (odd) operators $\digamma Z_{\mu \nu} F^{\mu \nu}\left(\digamma Z_{\mu \nu} \tilde{F}^{\mu \nu}\right)$ in the EW broken phase, see eq. (3.4). The angular dependence of the differential partonic cross-sections in the CP-even and CP-odd cases for $p p \rightarrow \digamma Z$ are

$$
\begin{aligned}
\frac{1}{\hat{\sigma}_{\mathrm{CP}-\text { odd }}} \frac{d \hat{\sigma}_{\mathrm{CP}-\text { odd }}}{d \cos \theta} & =\frac{3}{8}\left(1+\cos ^{2} \theta\right), \\
\frac{1}{\hat{\sigma}_{\mathrm{CP}-\text { even }}} \frac{d \hat{\sigma}_{\mathrm{CP}-\text { even }}}{d \cos \theta} & =\frac{3}{8}\left[\frac{1+\cos ^{2} \theta+8 M_{Z}^{2} \hat{s} / \lambda\left(\hat{s}, M_{Z}^{2}, M_{\digamma}^{2}\right)}{1+6 M_{Z}^{2} \hat{s} / \lambda\left(\hat{s}, M_{Z}^{2}, M_{\digamma}^{2}\right)}\right],
\end{aligned}
$$

where $\lambda(a, b, c) \equiv a^{2}+b^{2}+c^{2}-2(a b+b c+c a), \hat{s}$ is the partonic invariant mass squared of the system and $\theta$ is the angle between the direction of the $Z$ relative to the beam direction in the centre-of-mass reference frame. The angular dependence of the CP-odd case is purely $p$-wave, as illustrated in eq. (5.2), independently of the $Z$ velocity $\beta \equiv \sqrt{1-\left(M_{Z}+M\right)^{2} / \hat{s}}$. 

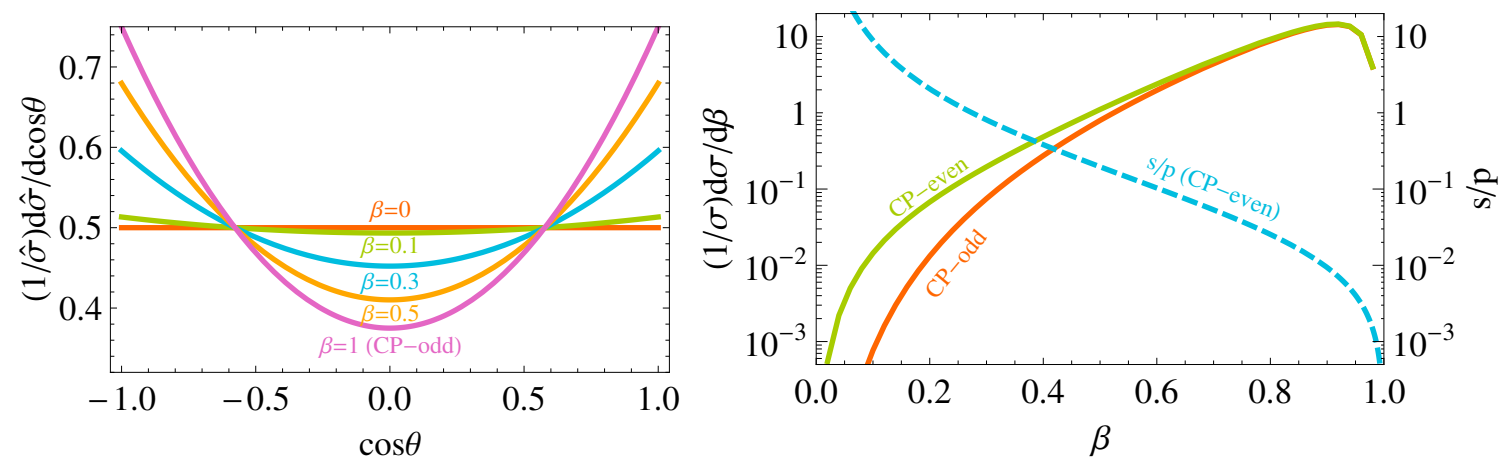

Figure 11. Left panel: angular dependence of the partonic $\digamma Z$ production cross-section in the centre-of-mass frame for the case of the CP-even operator $\left(c_{\gamma Z}\right)$ for different values of the $Z$ velocity $\beta$. The prediction for the $\mathrm{CP}$-odd operator $\left(\tilde{c}_{\gamma Z}\right)$ coincides with the curve at $\beta=1$. Right panel: normalised differential $\digamma Z$ associated production cross-section at $13 \mathrm{TeV}$ as a function of $\beta$, the $Z$ velocity in the centre-of-mass frame. We also show, on the right axis, the ratio $s / p$ of $s$-wave to $p$-wave contributions to the rate.

For this reason the largest $\mathrm{CP}$-even/CP-odd discrepancy is close to threshold, where the angular dependence is flat in the CP-even case, corresponding to $s$-wave dominance, as illustrated by the left panel of figure 11. The dependence on $\beta$ of the ratio between $s$ and $p$ wave contributions to the $\mathrm{CP}$-even cross section is illustrated in the right panel of figure 11.

At the same time the $p p \rightarrow \digamma Z$ production cross-sections for CP-even and CP-odd cases close to threshold differ,

$$
\frac{\hat{\sigma}_{\mathrm{CP}-\text { even }}}{\hat{\sigma}_{\mathrm{CP}-\text { odd }}}=\frac{c_{\gamma Z}^{2}}{\tilde{c}_{\gamma Z}^{2}}\left[1+\frac{6 M_{Z}^{2} \hat{s}}{\lambda\left(\hat{s}, M_{Z}^{2}, M_{\digamma}^{2}\right)}\right] .
$$

For a given $\Gamma(\digamma \rightarrow Z \gamma)$ this corresponds, after parton luminosity integration, to a $7 \%$ enhancement of the CP-even cross-section over the CP-odd one at the $13 \mathrm{TeV}$ LHC. This is shown in the right panel of figure 11.

The same angular dependence and cross-section ratio also appears in $\digamma Z$ production from the $\digamma Z Z$ couplings, with the replacement $c_{\gamma Z} \rightarrow c_{Z Z}$ and $\tilde{c}_{\gamma Z} \rightarrow \tilde{c}_{Z Z}$, as well as in $\digamma W$ production from the $\digamma W^{+} W^{-}$couplings, with the replacement $c_{\gamma Z} \rightarrow c_{\mathrm{WW}}$ and $\tilde{c}_{\gamma Z} \rightarrow \tilde{c}_{\mathrm{WW}}$. The CP properties of these interactions can also be probed using the angular distributions in $\digamma \rightarrow Z Z \rightarrow 4 f$ and $\digamma \rightarrow W^{+} W^{-} \rightarrow 4 f$ decays [96], or $\digamma \rightarrow \gamma^{*} \gamma^{*} \rightarrow 4 f$ as discussed above.

\subsection{EFT expansion and associated production}

An important aspect of associated production is that, contrary to resonant $\digamma$ production, the centre-of-mass energy of the parton process is not fixed and can vary in a wide range. In this context the question of the validity of our EFT expansion can become important and is complicated by the difficulty, contrary to resonant production, of associating a precise energy scale to the process. In fact, from an EFT perspective, operators of dimension 7 


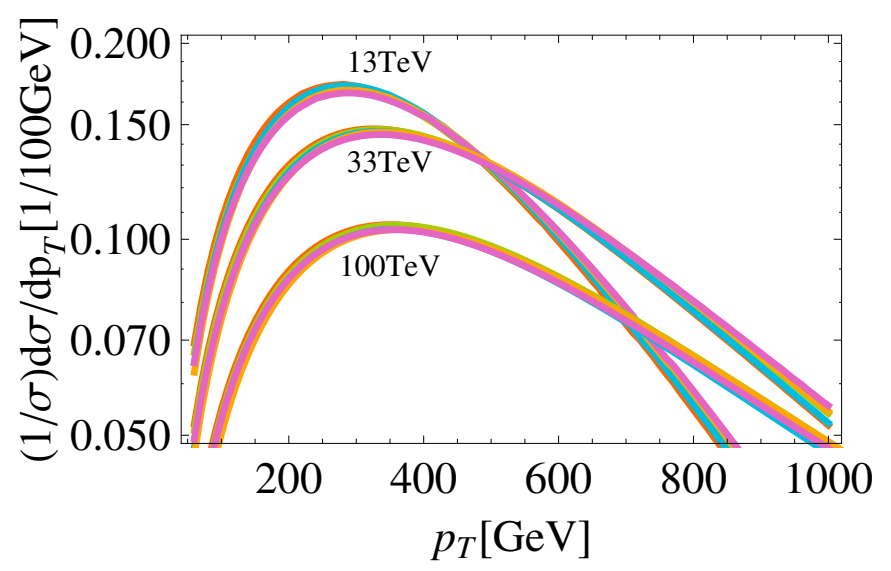

Figure 12. Transverse momentum distribution of $\digamma V$ associate production at $13 \mathrm{TeV}$ (highest peaked spectra), $33 \mathrm{TeV}$ (middle peaked spectra) and $100 \mathrm{TeV}$ (lowest peaked spectra) $p p$ collisions due to $c_{V V^{\prime}}$ interactions. The overlapping lines of different colours correspond to various EW bosons $V, V^{\prime}=\gamma, Z, W$.

or higher can also contribute to these processes (from our discussion before eq. (2.9) it is evident that there are no dimension- 6 operators linear in $\digamma$ ). Their effect (which has been ignored in our analysis) grows as $\sim\left|c_{i}^{(7)}\right|^{2} \hat{s}^{2} / \Lambda^{4}$ in the amplitude squared for $p p \rightarrow \digamma V$, $h$, and has to be compared with the leading dimension-5 contribution $\sim\left|c_{i}^{(5)}\right|^{2} \hat{s} / \Lambda^{2}$. While both effects grow (and eventually even cease making physical sense, when perturbative unitarity is violated [140]), their relative size crucially depends on $\Lambda$ and the Wilson coefficients $c_{i}^{(5)}$ and $c_{i}^{(7)}$ and cannot be determined without explicit UV assumptions.

Fortunately most of our analyses rely on the use of total cross sections, where the rapidly falling PDF distributions imply that the bulk of the EFT contributions are near threshold,as illustrated in figure 12 for $c_{B B}$ and $c_{W W}$ interactions. $^{8}$ In this kinematic region the centre-of-mass energy is close to that of single $\digamma$ production where the EFT description holds by construction, and the question of EFT validity can be expressed transparently in terms of the $M_{\digamma} / \Lambda$ expansion.

An illustrative example where the above-mentioned anomalous energy growth can be used to learn about the underlying theory, is the following. We compare a simple, renormalisable model where $\digamma=H^{0}$ is the neutral CP-even component of an additional EW doublet (and its couplings to SM fermions are dimensionless), with the scenario of eq. (2.5) where $\digamma$ is a singlet and its interactions $c_{\psi}$ are in fact non-renormalisable. We further assume that prompt $\digamma$ production is dominated by heavy quark annihilation. In the singlet model, scattering amplitudes for $q \bar{q} \rightarrow \digamma V_{L}(V=Z, W)$ are dominated by the contact interaction (last diagram in figure 9 ) and grow as $\sim \hat{s} / \Lambda^{2}$ at large energy. This can be seen in the hard $p_{T}$ spectra in hadronic collisions as shown in figure 13. In a renormalisable $\mathrm{SU}(2)_{L}$ invariant theory, on the other hand, this anomalous UV behaviour is regulated by the presence of additional degrees of freedom. In the case where $\digamma$ is the neutral compo-

\footnotetext{
${ }^{8}$ Note that even the discussion of the previous section, which relies on certain kinematic distributions, is most powerful near threshold $\beta \approx 0$.
} 

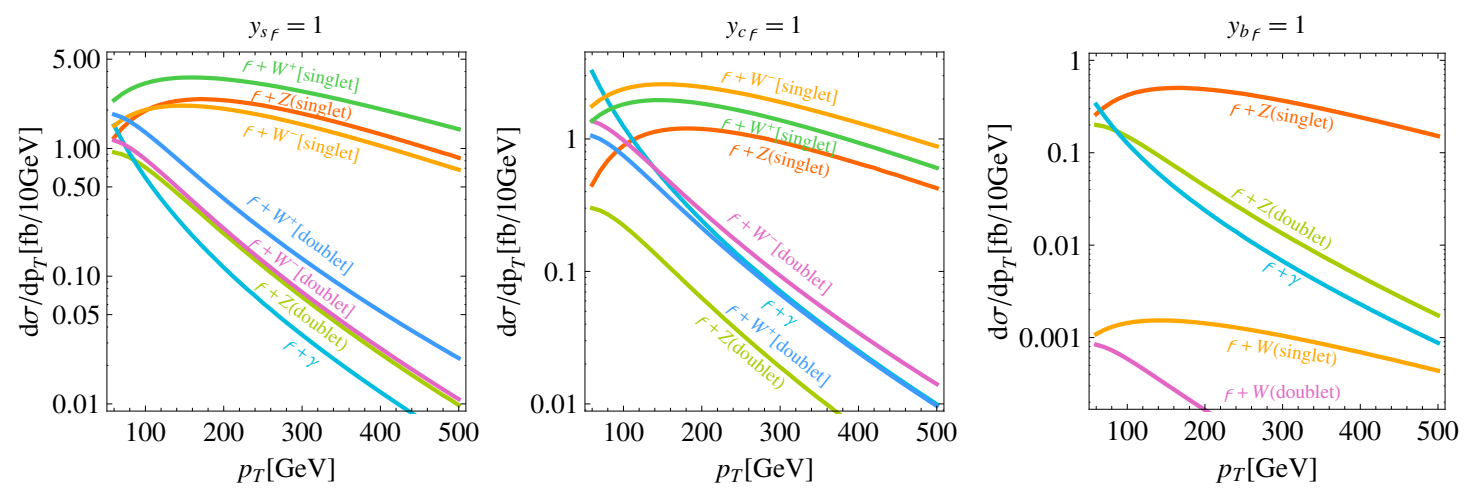

Figure 13. Transverse momentum distribution of $\digamma+V$ production at the $13 \mathrm{TeV}$ LHC due to $s \bar{s}$ (left panel), $c \bar{c}$ (center panel) and $b \bar{b}$ (right panel) annihilation induced by the $c_{s, c, b}$ couplings of eq. (2.5), that we rewrote in terms of $y_{q \digamma} \equiv c_{\psi} v / \Lambda$. The EW doublet results are obtained in the limit of degenerate doublet components.

nent of a $\mathrm{SU}(2)_{L}$ doublet, the $\digamma Z$ production now receives additional contributions from $s$-channel exchange of the other neutral components of the doublet, while $\digamma W$ production is regulated by the exchange of the associated charged scalars.

\section{$6 \quad p p \rightarrow \digamma \digamma$ : pair production}

\subsection{Effective theory parametrisation}

In the effective Lagrangian description of section $2.2, \digamma$ pair production receives contributions at different orders in the $1 / \Lambda$ expansion. In fact, starting already at the renormalisable level, the coupling $\lambda_{\digamma H}$ (which survives also in the limit of large separation of scales $\Lambda \gg M_{\digamma}$ ) generates $V_{L} V_{L} \rightarrow \digamma \digamma$ and $p p \rightarrow h^{*} \rightarrow \digamma \digamma$ via SM Higgs production channels. We have already shown in the table below eq. (2.1) that the $V_{L} V_{L}$ contribution to single production is small, and this result does not change substantially for pair-production so that this channel can be neglected. On the other hand, for $p p \rightarrow h^{*} \rightarrow \digamma \digamma$ we find

$$
\sigma(p p \rightarrow \digamma \digamma)=1.710^{-4} \lambda_{\digamma H}^{2} \mathrm{fb},
$$

where we neglected the subleading contribution coming from mixing with the Higgs and from dimension- 6 operators.

The presence of the relevant coupling $\kappa_{\digamma}$ in the renormalisable part of the Lagrangian eq. (2.3), implies that the rate for $p p \rightarrow \digamma^{*} \rightarrow \digamma \digamma$, with $\digamma^{*}$ produced by dimension-5 operators, can be thought to be formally of the same order in the EFT expansion; we write it as

$$
\sigma(p p \rightarrow \digamma \digamma)=\kappa_{\digamma}^{2} \frac{\mathrm{TeV}^{2}}{\Lambda^{2}}\left(270 c_{g g}^{2}+1.9 c_{u}^{2}+1.4 c_{d}^{2}+0.07 c_{s}^{2}+0.04 c_{c}^{2}+0.017 c_{b}^{2}\right) \mathrm{fb} .
$$

This implies that pair production can be reasonably large for realistic values of $\kappa_{\digamma}$

$$
\frac{\sigma_{\digamma \digamma}}{\sigma_{\digamma}}= \begin{cases}\left(\kappa_{\digamma} / 57\right)^{2} & g g \text { production } \\ \left(\kappa_{\digamma} / 63\right)^{2} & u \bar{u} \text { production } \\ \left(\kappa_{\digamma} / 88\right)^{2} & b \bar{b} \text { production }\end{cases}
$$


Note, however, that arguments based on vacuum stability restrict the coefficient of the cubic coupling to $\left|\kappa_{\digamma}\right|<6 \lambda_{\digamma}$ in the limit of large $\lambda_{\digamma}$, while vacuum meta-stability allows only for a small violation of this upper bound [58].

There are a number of reasons why this description in terms of an effective Lagrangian truncated at dimension- 5 might be incomplete in certain cases, and the next-order in $1 / \Lambda$ becomes necessary. First of all, it is plausible that the separation between $M_{\digamma}$ and $\Lambda$ is mild, as already suggested by the relatively large rates necessary to accommodate the observed excess. Secondly, it is possible that the $\digamma$ couplings to the underlying dynamics (e.g. additional particles in the loop) are sizeable and larger than the typical SM couplings. Finally, approximate global symmetries, preserved only by higher (in this case dimension6) order interactions, can lead to natural situations where the Wilson coefficients of the leading effects in the EFT expansion are actually suppressed. Examples of this are models where $\digamma$ is odd under an approximate $\mathrm{Z}_{2}$ symmetry, explicitly broken only by small effects, so that both $\kappa_{\digamma}$ and the full $\mathscr{L}_{5}$ in eq. (2.5) are suppressed. In any of these cases, the contribution from dimension-6 operators in eq. (2.9) (and in some cases of two insertions of dimension-5 operators), can be relevant. These give

$$
\begin{aligned}
\sigma(p p \rightarrow \digamma \digamma)= & \frac{\mathrm{TeV}^{4}}{\Lambda^{4}}\left[1.1 c_{g g}^{4}+2.1 c_{g g}^{2} c_{g g}^{(6)}+0.52 c_{g g}^{(6) 2}+73 c_{\digamma 3}^{2} c_{g g}^{2}+10^{-3}\left(0.0074 c_{\gamma \gamma}^{4}+\right.\right. \\
& \left.+0.099 c_{\gamma \gamma}^{2} c_{\gamma \gamma}^{(6)}+0.38 c_{\gamma \gamma}^{(6) 2}\right)+10^{-6}\left(11 c_{u}^{4}+6 c_{d}^{4}+0.25 c_{s}^{4}+0.14 c_{c}^{4}+0.05 c_{b}^{4}\right)+ \\
& \left.+10^{-3}\left(4.4 c_{u}^{(6) 2}+2.4 c_{d}^{(6) 2}+0.1 c_{s}^{(6) 2}+0.06 c_{c}^{(6) 2}+0.02 c_{b}^{(6) 2}\right)\right] \mathrm{pb}
\end{aligned}
$$

Interference in the quark diagrams is suppressed by the small quark masses and we have neglected for clarity the interference between these effects and those of eqs. (6.1), (6.2), assuming that either an approximate symmetry or a coupling hierarchy can account for a small $\kappa_{\digamma}$. In the limit where one production mode dominates we get the results shown in table 5 using

$$
\sigma(p p \rightarrow \digamma \digamma \rightarrow \gamma \gamma \digamma)=2 \sigma(p p \rightarrow \digamma \rightarrow \gamma \gamma) \frac{\sigma(p p \rightarrow \digamma \digamma)}{\sigma(p p \rightarrow \digamma)}
$$

and

$$
\sigma(p p \rightarrow \digamma \digamma \rightarrow 4 \gamma)=\sigma(p p \rightarrow \digamma \digamma)\left(\frac{\sigma(p p \rightarrow \digamma \rightarrow \gamma \gamma)}{\sigma(p p \rightarrow \digamma)}\right)^{2}
$$

and having fixed $\sigma(p p \rightarrow \digamma \rightarrow \gamma \gamma)=3 \mathrm{fb}$, which is the experimentally favoured value as extracted from a fit to the preferred cross sections of table 2, under the assumption of production from gluon fusion.

The present experimental bounds on $p p \rightarrow \digamma \digamma$ pair production at $\sqrt{s}=8 \mathrm{TeV}$ are listed in table 6 . Using present data, the $4 \gamma$ limit can easily be improved down to $0.1 \mathrm{fb}$ or better with a dedicated search. The $4 j$ bound implies

$$
\sigma(p p \rightarrow \digamma \digamma \rightarrow j j \gamma \gamma)<\frac{\Gamma_{\gamma \gamma}}{\Gamma_{j j}} \times 0.2 \mathrm{pb}, \quad \sigma(p p \rightarrow \digamma \digamma \rightarrow \gamma \gamma \gamma \gamma)<\left(\frac{\Gamma_{\gamma \gamma}}{\Gamma_{j j}}\right)^{2} \times 0.1 \mathrm{pb} .
$$

We see that, unless $\digamma$ is produced from $\gamma \gamma$ partons, detectable cross sections for $\digamma \digamma$ production need $c_{\wp}^{(6)} \gg c_{\wp}$ and a not too large $\Lambda$. Large $c_{\wp}^{(6)}$ are in some cases rather 


\begin{tabular}{|c|c|c|}
\hline$\digamma$ couples to & $\sigma_{\digamma \digamma} / \sigma_{\digamma}=\sigma_{\gamma \gamma \digamma} / 2 \sigma_{\gamma \gamma}$ & $\sigma_{4 \gamma} / \sigma_{\gamma \gamma}$ \\
\hline$b \bar{b}$ & $0.015 \%(\mathrm{TeV} / \Lambda)^{2}\left(c_{b}^{(6)} / c_{b}\right)^{2}$ & $3.6 \times 10^{-6}(\mathrm{TeV} / \Lambda)^{2}\left(c_{b}^{(6)} / c_{b}\right)^{2}$ \\
$c \bar{c}$ & $0.021 \%(\mathrm{TeV} / \Lambda)^{2}\left(c_{c}^{(6)} / c_{c}\right)^{2}$ & $2.1 \times 10^{-6}(\mathrm{TeV} / \Lambda)^{2}\left(c_{c}^{(6)} / c_{c}\right)^{2}$ \\
$s \bar{s}$ & $0.023 \%(\mathrm{TeV} / \Lambda)^{2}\left(c_{s}^{(6)} / c_{s}\right)^{2}$ & $1.5 \times 10^{-6}(\mathrm{TeV} / \Lambda)^{2}\left(c_{s}^{(6)} / c_{s}\right)^{2}$ \\
$u \bar{u}$ & $0.058 \%(\mathrm{TeV} / \Lambda)^{2}\left(c_{u}^{(6)} / c_{u}\right)^{2}$ & $0.23 \times 10^{-6}(\mathrm{TeV} / \Lambda)^{2}\left(c_{u}^{(6)} / c_{u}\right)^{2}$ \\
$d \bar{d}$ & $0.050 \%(\mathrm{TeV} / \Lambda)^{2}\left(c_{d}^{(6)} / c_{d}\right)^{2}$ & $0.31 \times 10^{-6}(\mathrm{TeV} / \Lambda)^{2}\left(c_{d}^{(6)} / c_{d}\right)^{2}$ \\
$G G$ & $0.13 \%(\mathrm{TeV} / \Lambda)^{2}\left(c_{g g}^{(6)} / c_{g g}\right)^{2}$ & $0.006 \times 10^{-6}(\mathrm{TeV} / \Lambda)^{2}\left(c_{g g}^{(6)} / c_{g g}\right)^{2}$ \\
$\gamma \gamma$ & $1.9 \%(\mathrm{TeV} / \Lambda)^{2}\left(c_{\gamma \gamma}^{(6)} / c_{\gamma \gamma}\right)^{2}$ & $2.9 \times 10^{-3}(\mathrm{TeV} / \Lambda)^{2}\left(c_{\gamma \gamma}^{(6)} / c_{\gamma \gamma}\right)^{2}$ \\
\hline
\end{tabular}

Table 5. Predictions for leading order contributions to pair production of the resonance $\digamma$ at $\sqrt{s}=13 \mathrm{TeV}$.

\begin{tabular}{|c|ccc|}
\hline & $\sigma(p p \rightarrow \digamma \digamma \rightarrow j j j j)$ & $\sigma(p p \rightarrow \digamma \digamma \rightarrow \gamma \gamma j j)$ & $\sigma(p p \rightarrow \digamma \digamma \rightarrow \gamma \gamma \gamma \gamma)$ \\
\hline Bound at LHC, $\sqrt{s}=8 \mathrm{TeV}$ & $<0.1 \mathrm{pb}[141]$ & - & $\lesssim 26 \mathrm{fb}[142]$ \\
Background at $\sqrt{s}=8 \mathrm{TeV}$ & see $[141]$ & $\sim 0.07 \mathrm{fb}$ & $\sim 4 \mathrm{ab}$ \\
Background at $\sqrt{s}=13 \mathrm{TeV}$ & $5 \times[141]$ & $\sim 0.2 \mathrm{fb}$ & $\sim 8 \mathrm{ab}$ \\
\hline
\end{tabular}

Table 6. Summary of $p p \rightarrow \digamma \digamma$ searches. The $4 \gamma$ search [142] was not optimized for double production of resonances. Furthermore [143] finds $\sigma(p p \rightarrow \digamma \digamma \rightarrow t \bar{t} t \bar{t})<70 \mathrm{fb}$ with an expected bound of $<20 \mathrm{fb}$.
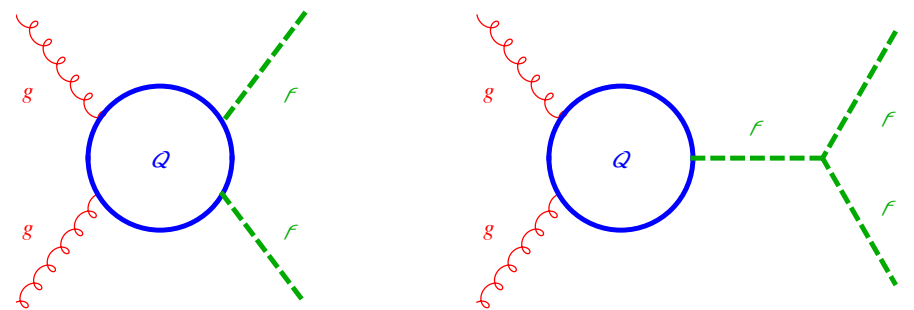

Figure 14. Feynman diagrams contributing to pair production of the $750 \mathrm{GeV}$ resonance.

plausible, in particular for initial-state quarks $\wp=q$ where these couplings can be generated at tree level in a UV-complete underlying model. An explicit realization of this is a model with additional heavy vector-like quarks $\mathcal{Q}$ with couplings $y_{\digamma} \digamma \overline{\mathcal{Q}} q$ and $y_{\digamma}^{\prime} \digamma \overline{\mathcal{Q}} \mathcal{Q}$ larger than the Yukawa couplings $y_{H} H \overline{\mathcal{Q}} q$. In such theories the ratio of Wilson coefficients $c_{q}^{(6)} / c_{q}^{(5)} \sim$ $y_{\digamma}^{\prime}$ (neglecting a small contribution proportional to the SM Yukawa $y_{q}^{\mathrm{SM}}$ ), can be large.

\subsection{Model computation in low energy theorem approximation}

To better appreciate the prospects offered by $\digamma$ pair prduction, it is instructive to consider an explicit renormalizable model, where $\digamma \rightarrow \gamma \gamma$ is mediated by a loop of heavy vector-like 
fermions $\mathcal{Q}_{r}$ coupled to $\digamma$ as

$$
\mathscr{L}_{\mathcal{Q}}=\sum_{r} \overline{\mathcal{Q}}_{r}\left(i \not D-M_{r}-y_{r} \digamma\right) \mathcal{Q}_{r}
$$

Throughout we will consider these fermions as coloured and/or carrying hypercharge, but for simplicity we do not consider fermions with $\mathrm{SU}(2)_{W}$ charge. $p p \rightarrow \digamma \digamma$ is unavoidably obtained by attaching twice $\digamma$ to the loop as well as by a possible cubic $\digamma^{3}$ term, as depicted by the Feynman diagrams in figure 14. In the limit that the new fermions are relatively heavy, $2 M_{r} \gtrsim M_{\digamma}$, we may employ the low energy theorem (LET) to determine the dominant coupling to gluons and photons [144-150]. To see this we may write the contribution of any new massive coloured field to the QCD $\beta$-function as $\Delta \beta_{3}=\Delta b_{3} g_{3}^{3} / 16 \pi^{2}$ where, as an example, for $N_{r}$ new coloured fermions of Casimir $I_{r}$ (normalised such that $I_{r}=1 / 2$ for the fundamental representation) we have $\Delta b_{3}=4 N_{r} I_{r} / 3$. Writing the mass of this field as $M(\digamma)$ (which explicitly includes $\digamma$ as a background field) and running the gauge coupling from a high scale $\Lambda$ to some low scale $\mu$, the gauge kinetic terms pick up a correction at the mass threshold, given by $\mathscr{L}_{\mathrm{LET}}=\Delta b_{3} \alpha_{3} / 8 \pi \ln (M(\digamma) / \mu) G^{a \mu \nu} G_{\mu \nu}^{a}$. The case for QED is analogous. This derivation is general for any field whose mass depends on $\digamma$. For our simple example case it gives

$$
\mathscr{L}_{\mathrm{LET}}=\sum_{r}\left(I_{r} N_{r} \frac{\alpha_{3}}{6 \pi} G^{a \mu \nu} G_{\mu \nu}^{a}+q_{r}^{2} N_{r}^{\prime} \frac{\alpha}{6 \pi} F^{\mu \nu} F_{\mu \nu}\right) \ln \left(1+\frac{\digamma}{v_{r}}\right) .
$$

where the loop contribution also includes $N_{r}^{\prime}$ fermionic vector-like components with electric charge $q_{r}$, and we have defined

$$
v_{r} \equiv \frac{M_{r}}{y_{r}} .
$$

Expanding the logarithm provides the low energy theorem (LET) description of multiple scalar production from gluon or photon fusion. ${ }^{9}$ In fact, we can see that in the absence of a scalar self coupling the pair production amplitude is related to the single production amplitude simply by a factor of $1 / v_{r}$.

To make our expressions more transparent, we limit our discussion to the case of $N_{Q}$ copies of identical electrically-neutral coloured fermions with Casimir $I_{Q}$ and $N_{L}$ copies of colourless fermions with charge $q_{L}$. We also take masses and couplings universal in the two sectors, which are then described by the two scales $v_{Q} \equiv M_{Q} / y_{Q}$ and $v_{L} \equiv M_{L} / y_{L}$. The extension to general fermion representations is completely straightforward and can be expressed in terms of effective $v_{Q}$ and $v_{L}$. In particular, heavy fermions with both colour and electric charge simultaneously contribute to both $v_{Q}$ and $v_{L}$.

Using this description the decay widths of the particle $\digamma$ into gluon and photon pairs are

$$
\Gamma_{g g}=\frac{\alpha_{3}^{2} N_{Q}^{2} I_{Q}^{2} M_{\digamma}^{3}}{18 \pi^{3} v_{Q}^{2}}, \quad \Gamma_{\gamma \gamma}=\frac{\alpha^{2} q_{L}^{4} N_{L}^{2} M_{\digamma}^{3}}{144 \pi^{3} v_{L}^{2}}
$$

\footnotetext{
${ }^{9} \mathrm{~A}$ translation to the operators in eqs. (2.5) and (2.9) is $c_{g g} / \Lambda=I_{r} N_{r} /\left(12 \pi^{2} v_{r}\right)$, and $c_{g g}^{(6)} / \Lambda^{2}=$ $-I_{r} N_{r} /\left(24 \pi^{2} v_{r}^{2}\right)$.
} 
The corresponding single production cross section $\sigma(p p \rightarrow \digamma)$, initiated by gluon and photon annihilations, is

$$
\sigma(p p \rightarrow \digamma)=\frac{1}{s M_{\digamma}}\left[\Gamma_{g g} C_{g g}\left(\frac{M_{\digamma}^{2}}{s}\right)+\Gamma_{\gamma \gamma} C_{\gamma \gamma}\left(\frac{M_{\digamma}^{2}}{s}\right)\right],
$$

where, as defined in [7],

$$
C_{g g}\left(\frac{\hat{s}}{s}\right)=\frac{\pi^{2}}{8} \int_{\hat{s} / s}^{1} \frac{d x}{x} g(x) g\left(\frac{\hat{s}}{s x}\right), \quad C_{\gamma \gamma}\left(\frac{\hat{s}}{s}\right)=8 \pi^{2} \int_{\hat{s} / s}^{1} \frac{d x}{x} \gamma(x) \gamma\left(\frac{\hat{s}}{s x}\right),
$$

and $s(\hat{s})$ is the proton (parton) squared centre-of-mass energy. ${ }^{10}$

The pair-production cross section $p p \rightarrow \digamma \digamma$ also depends on the value of the possible cubic interaction, $\kappa_{F} M_{\digamma} \digamma^{3}$ in the potential of eq. (2.4). It is convenient here to rewrite it as $\kappa_{F}=\kappa M_{\digamma} / 2 v_{Q}$. Higher order terms, such as the quartic coupling, are not relevant for this study. In the LET limit, after partonic integration, the colour and spin averaged total pair production cross section at the LHC is

$$
\sigma(p p \rightarrow \digamma \digamma)=\frac{1}{8 \pi^{2} M_{\digamma}}\left[\frac{\Gamma_{g g}}{v_{Q}^{2}} C_{g g}^{\prime}\left(\frac{M_{\digamma}^{2}}{s}\right)+\frac{\Gamma_{\gamma \gamma}}{v_{L}^{2}} C_{\gamma \gamma}^{\prime}\left(\frac{M_{\digamma}^{2}}{s}\right)\right],
$$

where the weighted partonic luminosities, including the kinematic dependence from the two interfering diagrams and the phase space factors, are

$$
\begin{aligned}
& C_{g g}^{\prime}(z)=\int_{4 z}^{1} d y C_{g g}(y) \frac{y}{4 z} \sqrt{1-\frac{4 z}{y}}\left[1-\kappa \frac{3 z}{y-z}\right]^{2}, \\
& C_{\gamma \gamma}^{\prime}(z)=\int_{4 z}^{1} d y C_{\gamma \gamma}(y) \frac{y}{4 z} \sqrt{1-\frac{4 z}{y}}\left[1-\kappa \frac{v_{L}}{v_{Q}} \frac{3 z}{y-z}\right]^{2} .
\end{aligned}
$$

If we assume that gluon-initiated production dominates over photon-initiated production, in the LET limit the ratio of the cross sections of double to single $\digamma$ production depends on the new fermion content and quantum numbers only through the scale $v_{Q}$, and is simply given by

$$
\frac{\sigma(p p \rightarrow \digamma \digamma)}{\sigma(p p \rightarrow \digamma)}=\frac{M_{\digamma}^{2}}{v_{Q}^{2}} \zeta(\kappa), \quad \zeta(\kappa) \equiv \frac{s}{8 \pi^{2} M_{\digamma}^{2}} \frac{C_{g g}^{\prime}\left(M_{\digamma}^{2} / s\right)}{C_{g g}\left(M_{\digamma}^{2} / s\right)} .
$$

The solid line in figure 15 shows $\zeta(\kappa)$ as a function of the $\digamma$ self coupling, using the LET result in eq. (6.16). For comparison we also show the values of $\zeta(\kappa)$ determined by the full one-loop calculation for various masses $M_{Q}$ of the new fermion (with $N_{Q}=1$ and $\left.I_{Q}=1 / 2\right)$. A good fit of the LET result is

$$
\zeta(\kappa) \approx 3.2 \times 10^{-4}\left(1-\kappa+0.3 \kappa^{2}\right) .
$$

Equations (6.16) and (6.17) allow for a quick estimate of the pair production cross section, assuming that the single production cross section is known. For example, if we want to

\footnotetext{
${ }^{10}$ The parton distribution functions also depend on the factorisation scale, however we have suppressed this variable in the equations above and taken the factorisation scale as $\mu=\sqrt{\hat{s}}$ throughout.
} 


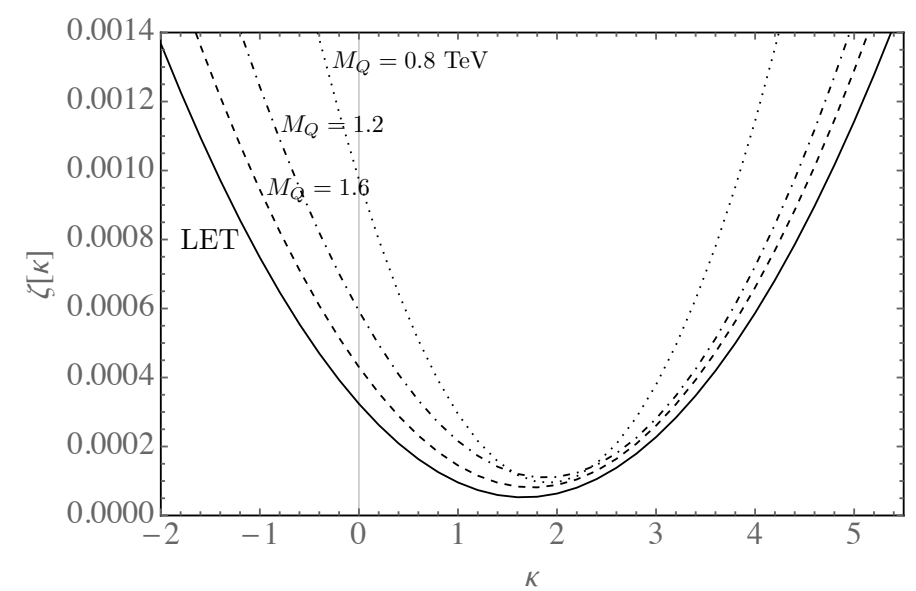

Figure 15. The function $\zeta(\kappa)$ that determines the ratio between the pair and single production cross section for gluon-initiated processes, as defined in eq. (6.16). The solid line refers to the LET result, while the other lines show the result when finite fermion mass form factors are included in the calculation, as discussed in section 6.3.

reproduce the experimentally favoured value $\sigma(p p \rightarrow \digamma \rightarrow \gamma \gamma) \approx 3 \mathrm{fb}$ for gluon-initiated production, we find $v_{Q}=N_{Q} I_{Q} \sqrt{\mathrm{BR}_{\gamma \gamma}} 3.5 \mathrm{TeV}$ and eq. (6.16) leads to

$$
\sigma(p p \rightarrow \digamma \digamma)=4.7 \times 10^{-5} \mathrm{fb} \frac{1-\kappa+0.3 \kappa^{2}}{\left(N_{Q} I_{Q} \mathrm{BR}_{\gamma \gamma}\right)^{2}} .
$$

Taking into account the branching ratios we have

$$
\begin{aligned}
& \sigma(p p \rightarrow \digamma \digamma \rightarrow \gamma \gamma \gamma \gamma)=4.7 \times 10^{-5} \mathrm{fb} \frac{1-\kappa+0.3 \kappa^{2}}{\left(N_{Q} I_{Q}\right)^{2}}, \\
& \sigma(p p \rightarrow \digamma \digamma \rightarrow g g g g)=4.7 \times 10^{-5} \mathrm{fb} \frac{1-\kappa+0.3 \kappa^{2}}{\left(N_{Q} I_{Q}\right)^{2}}\left(\frac{\Gamma_{g g}}{\Gamma_{\gamma \gamma}}\right)^{2}, \\
& \sigma(p p \rightarrow \digamma \digamma \rightarrow \gamma \gamma g g)=9.4 \times 10^{-5} \mathrm{fb} \frac{1-\kappa+0.3 \kappa^{2}}{\left(N_{Q} I_{Q}\right)^{2}} \frac{\Gamma_{g g}}{\Gamma_{\gamma \gamma}} .
\end{aligned}
$$

having assumed that production is dominated by gluon fusion.

In figure 16 we take into account diphoton-initiated pair production, and show the results of the low energy theorem prediction for $\sigma(p p \rightarrow \digamma \digamma \rightarrow \gamma \gamma j j)$ and $\sigma(p p \rightarrow \digamma \digamma \rightarrow$ $\gamma \gamma \gamma \gamma$ ), assuming a vanishing $\digamma^{3}$ coupling for a benchmark model of two triplets of coloured fermions and three leptons with unit charge. This choice of benchmark parameters is motivated by figure 5 of [7], such that the required ranges of $\Gamma_{\gamma \gamma}$ and $\Gamma_{g g}$ may be found for reasonably perturbative couplings, particularly in the narrow width scenario. This can be seen by comparing with the required values of $v_{Q}$ and $v_{L}$, which show that for $M_{Q}$ and $M_{L}$ in the range of 100's GeV, the required Yukawa coupling becomes non-perturbative only at the extreme ranges of the parameter space, when the width is becoming large.

For this benchmark model, experimental constraints on $\sigma(p p \rightarrow \digamma \digamma \rightarrow 4 g)$ already place relevant bounds on the parameter space. In some regions of allowed parameter space the $\sigma(p p \rightarrow \digamma \digamma \rightarrow \gamma \gamma g g)$ final state may be observable in the future with $\sim 300 \mathrm{fb}^{-1}$ of 


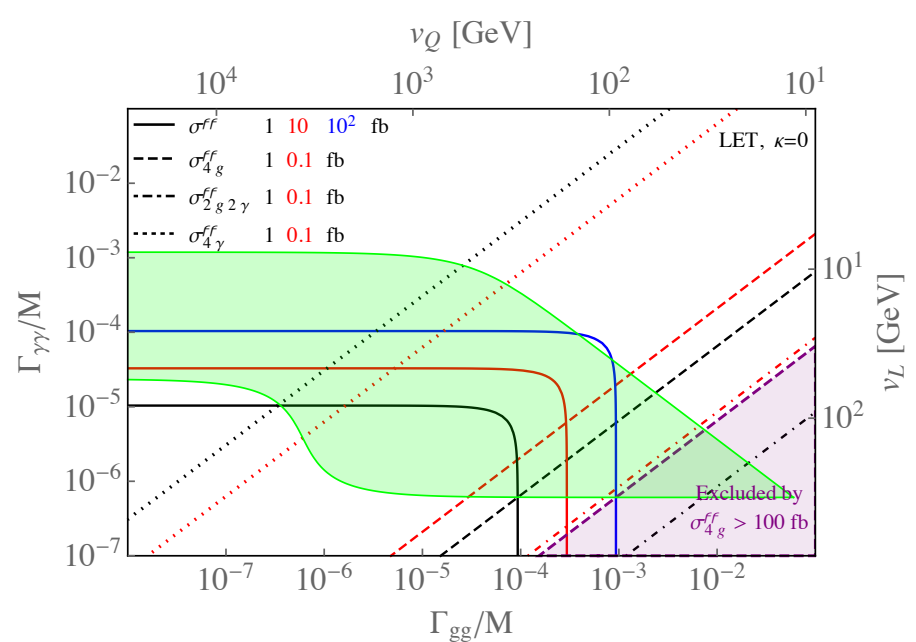

Figure 16. Within the green region one can reproduce the di-photon excess $\sigma(p p \rightarrow \gamma \gamma) \approx 3 \mathrm{fb}$ at the $13 \mathrm{TeV}$ LHC. Contours of constant $\sigma(p p \rightarrow \digamma \digamma)$ are shown as solid lines, $\sigma(p p \rightarrow \digamma \digamma \rightarrow 4 g)$ as dashed, $\sigma(p p \rightarrow \digamma \digamma \rightarrow \gamma \gamma g g)$ as dotdashed, and $\sigma(p p \rightarrow \digamma \digamma \rightarrow 4 \gamma)$ as dotted. These cross sections are computed using LET with two triplets of coloured fermions and three leptons with unit charge, and the $\digamma^{3}$ coupling is set to zero. The required scales $v_{Q}=M_{Q} / y_{Q}$ and $v_{L}=M_{L} / y_{L}$ are also shown.

integrated luminosity. However, in much of the parameter space the cross section for this process is too small. In the upper left hand plane for much of the parameter space $\sigma(p p \rightarrow$ $\digamma \digamma \rightarrow 4 \gamma)>0.1 \mathrm{fb}$, also suggesting that this channel could be observable. However, in most of the region where this is observable the dominant production mode is from photon fusion, and this region is disfavoured due to the reduced increase in single production cross section going from $8 \mathrm{TeV}$ to $13 \mathrm{TeV}$.

In figure 17 we project the different cross sections along the narrow width line (lower edge of the green region in figure 16) and along the $\Gamma / M=0.06$ line (upper edge in figure 16). There are a number of interesting features. As before, it is clear that dijet pair production places an interesting constraint on the parameter space. Second, as $\Gamma_{g g}$ is reduced, then $\Gamma_{\gamma \gamma}$ must be increased as we go along either boundary. Correspondingly, the diphoton contribution to single and pair production increases. Since partonic gluons are softer than partonic photons, the photon fusion contribution to $p p \rightarrow \digamma \digamma$ is relatively more important, with respect to the gluon fusion, than the photon fusion contribution to $p p \rightarrow \digamma$. This means that there are regions of parameter space where $p p \rightarrow \digamma$ is dominated by gluon fusion, giving good consistency between $8 \mathrm{TeV}$ and $13 \mathrm{TeV}$ data, and at the same time $p p \rightarrow \digamma \digamma$ is dominated by photon fusion.

Another feature worth highlighting is that as one goes to very small $\Gamma_{g g}$ and $\Gamma_{\gamma \gamma}$ is increased, the inclusive pair production cross section may become larger than the single production cross section, while, even in a strongly-coupled model, one expects that it should be $10-50$ times smaller because of the reduced parton luminosity. This is particularly noticeable for the $\Gamma / M=0.06$ assumption. This is not, however, physical. It is rather signalling the breakdown of perturbation theory since the value of $\Gamma_{\gamma \gamma}$ required to explain 

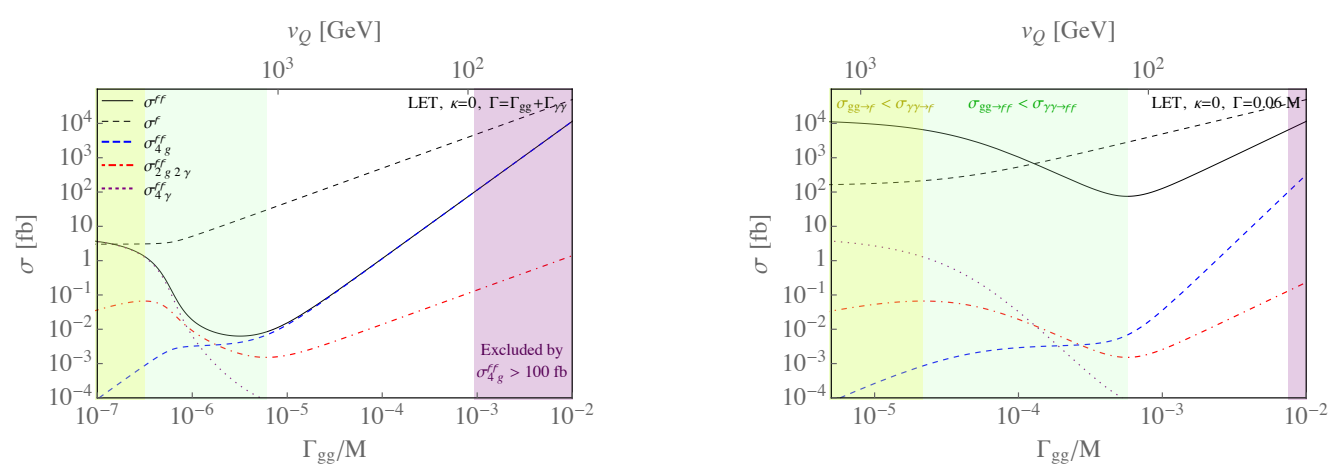

Figure 17. Cross sections as a function of $\Gamma_{g g} / M$ once the requirement of $\sigma(p p \rightarrow \digamma \rightarrow 2 \gamma)=3 \mathrm{fb}$ has been imposed. The narrow width assumption is shown on the left panel (corresponding to travelling along the lower boundary of the green region in figure 16) and a broader resonance is shown on the right panel (travelling along the upper boundary in figure 16).

the excess is becoming so large that for the benchmark parameters chosen and for fixed $v_{L}$ the implied Yukawa coupling to charged fermions are becoming too large. Thus in the region where pair production is comparable or larger than single production the predicted rates for either should not be trusted. More specifically, the ratio of pair to single production cross sections scales approximately as

$$
\sigma_{\digamma \digamma} / \sigma_{\digamma} \propto\left(y M_{\digamma} / 4 \pi M_{Q}\right)^{2} .
$$

For the LET description to remain valid we require $M_{Q} \gtrsim M_{\digamma}$ : in the strongly coupled limit, $y \gg 1$, it is possible to have $v_{\digamma} \lesssim M_{\digamma}$ while the LET description remains valid, at the cost of approaching the non-perturbativity limit, as can be seen in figure 17.

Finally we note that in regions of parameter space where gluon fusion dominates the production by far the largest observable final state is $p p \rightarrow \digamma \digamma \rightarrow 4 g$. The cross section for $p p \rightarrow \digamma \digamma \rightarrow \gamma \gamma g g$ stays approximately in the region $10^{-3} \rightarrow 10^{-1} \mathrm{fb}$.

In summary, pair-production is experimentally interesting. For the benchmark scenario considered here we find that $\sigma(p p \rightarrow \digamma \digamma \rightarrow 4 g) \gtrsim 1 \mathrm{fb}$ provided that $\Gamma_{g g} / M \gtrsim 9.5 \times 10^{-5}$ $\left(v_{Q} \lesssim 290 \mathrm{GeV}\right)$, and for $\sigma(p p \rightarrow \digamma \digamma \rightarrow 2 g 2 \gamma) \gtrsim 0.1 \mathrm{fb}$ provided that $\Gamma_{g g} / M \gtrsim 7 \times 10^{-4}$ $\left(v_{Q} \lesssim 100 \mathrm{GeV}\right)$. For other representations these numbers will be different, however it is clear that for $\mathcal{O}(1)$ Yukawa couplings the model may accommodate the observed excess while predicting $p p \rightarrow \digamma \digamma \rightarrow 4 g$ and $p p \rightarrow \digamma \digamma \rightarrow 2 g 2 \gamma$ rates within reach of the LHC. Whether these signals are observable will depend on the SM background, which is discussed in section 6.7.

\subsection{Full computation beyond the LET approximation}

For large portions of the relevant parameter space the LET description may not be valid as either very large Yukawa couplings may be required (especially when the vector-like fermions are very massive $M_{Q} \gg M_{\digamma}$ ) or the low-energy approximation breaks down, because $M_{Q} \lesssim M_{\digamma}$. This second problem can be solved by including the full one-loop 

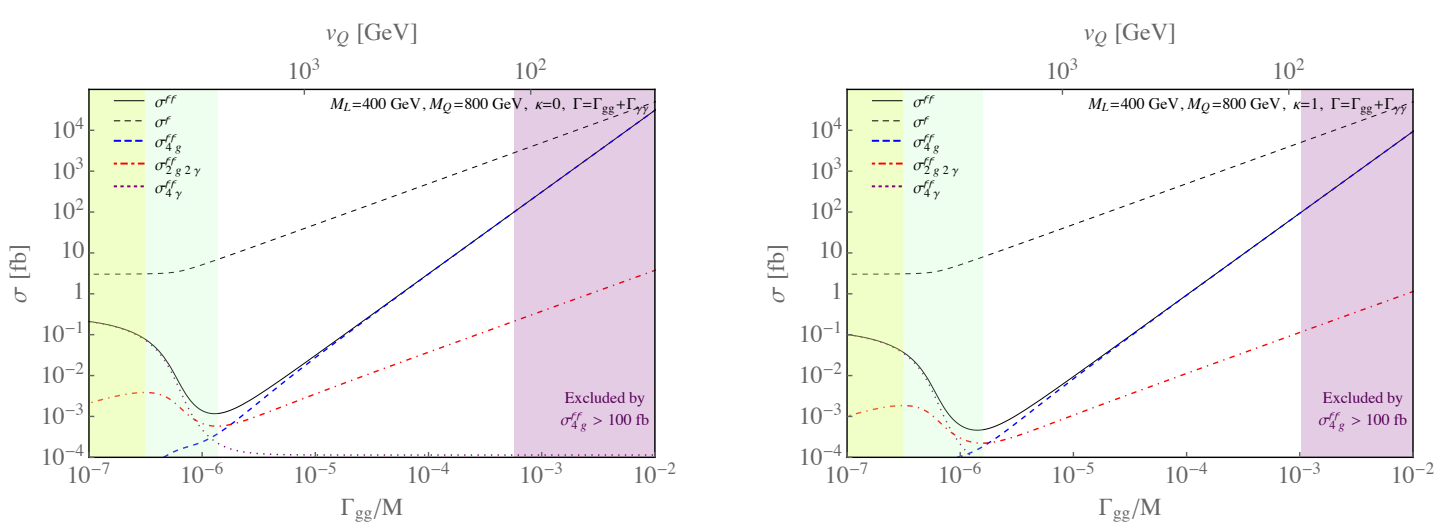

Figure 18. As in the narrow width case in figure 17, but for massive fermion loops with the full form factor included. The left panel assumes vanishing self coupling $(\kappa=0)$, and the right a Higgs-like self-coupling $(\kappa=1)$.

result, which is at first order in perturbation theory, so will still break down for large Yukawa couplings, but is all orders in the heavy fermion masses, allowing the study of scenarios with $M_{Q} \sim M_{\digamma}$. The pair production of Higgs-like scalars at one loop from virtual fermions has been studied for all fermion masses for some time [151, 152] and later with QCD corrections [153-155]. As the full one loop expressions are lengthly we refer the reader to [152] where the relevant formulæ are conveniently presented.

In figure 18 we show contours of constant pair production cross section for the benchmark scenario, now with lepton mass $M_{L}=400 \mathrm{GeV}$ and quark mass $M_{Q}=800 \mathrm{GeV}$, for the case of a narrow width. We show the results for vanishing self coupling $(\kappa=0)$, and for a Higgs-like self-coupling $(\kappa=1)$. As can be seen, including the full one loop fermion mass dependence leads to relevant quantitative differences from the LET approximation of figure 16, while the qualitative aspects are similar. When it occurs, the breakdown of the description only occurs as large Yukawa couplings are required, as all mass effects are included.

\subsection{Pair production of a pseudo-scalar resonance}

We may also consider the single and pair production of a pseudoscalar at one loop due to interactions with heavy vector-like fermions

$$
\mathcal{L}=\tilde{y} \digamma \overline{\mathcal{Q}} i \gamma_{5} \mathcal{Q}+M_{Q} \overline{\mathcal{Q}} \mathcal{Q}
$$

In this case the single production cross section only differs from the scalar case by an additional factor of $c_{P}=9 / 4$. For pair production the cross section is identical to pair production of the scalar, with the additional simplification that the cubic coupling $\kappa$ vanishes in the CP-symmetric limit. An example plot for the pseudoscalar is shown in figure 19.

In composite models $\digamma$ can be a pseudo-scalar analogous to the $\eta$ in QCD: a Goldstone boson of an accidental global symmetry spontaneously broken by the new interaction that becomes strong at $\Lambda_{\mathrm{TC}}$. Its linear and quadratic couplings are $\digamma G \tilde{G}$ and $\digamma^{2} G^{2}$ : the latter operator breaks the global symmetry and thereby its coefficient is suppressed by $M_{\digamma}^{2} / \Lambda_{\mathrm{TC}}^{2}$. 


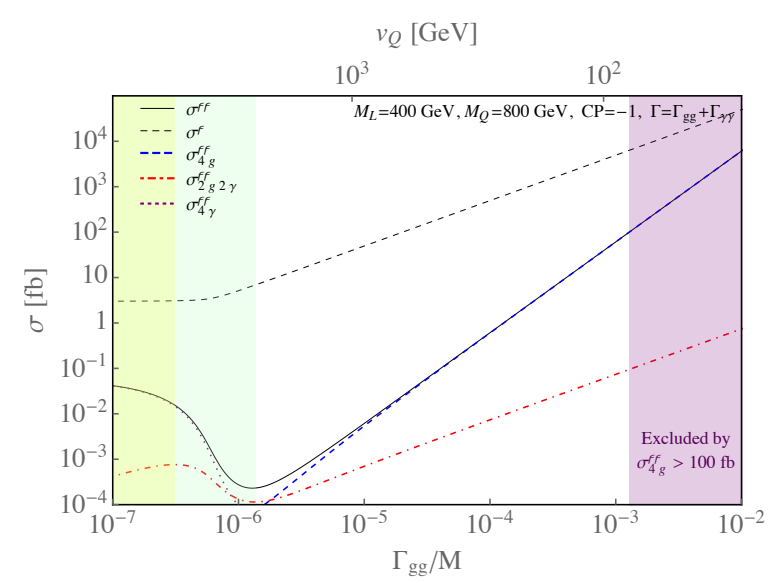

Figure 19. As in figure 18, but for a pseudo-scalar resonance. In the CP-symmetric limit the self-coupling vanishes.

\subsection{Decorrelating single and pair production}

From section 6.2 it may appear that in complete models a precise correlation between single and pair production operators is generically expected. On the other hand, we saw in section 6.4 that for a pseudoscalar the correlation between the two changes. This is related to the fact that, based on CP symmetry, one would only expect odd powers of $\digamma$ coupled to $G \widetilde{G}$, and even powers coupled to $G G$. However, the decorrelation of single and double production may be even greater in the presence of other global symmetries.

To illustrate this let us consider a model where $\digamma$ is odd under a $Z_{2}$ symmetry. In this case one would only expect even powers of $\digamma$ in the effective theory, thus single production is forbidden. One can introduce single production, but this would be controlled by a parameter which breaks the $Z_{2}$ and may thus be small. In this way, pair production may be enhanced relative to single production in the presence of additional approximate global symmetries.

As an example, consider the model of eq. (6.8) with two flavours of heavy quark $Q_{1,2}$ with interactions

$$
\mathscr{L}_{Q_{1,2}} \supset M_{1} \bar{Q}_{1} Q_{1}+M_{2} \bar{Q}_{2} Q_{2}+y_{1,2} \digamma \bar{Q}_{1} Q_{2}+y_{2,1} \digamma \bar{Q}_{2} Q_{1}
$$

Using the LET and keeping the dependence of the fermion masses on $\digamma$ we obtain a contribution to the $G G$ coupling $\propto\left(\log \left(M_{+}(\digamma) / \Lambda\right)+\log \left(M_{-}(\digamma) / \Lambda\right)\right)$ where $M_{ \pm}$are the two mass eigenstates, in the presence of a background $\digamma$ field value, and $\Lambda$ is a high energy scale that drops out when expanding in powers of $\digamma$. In the end the effective coupling to the $G G$ operator is

$$
\mathscr{L}_{\mathrm{LET}}=I_{r} N_{r}\left(0 \times \digamma-\frac{y_{1,2} y_{2,1} \digamma^{2}}{M_{1} M_{2}}+\mathcal{O}\left(\digamma^{4}\right)\right) \frac{\alpha_{3}}{6 \pi} G^{a \mu \nu} G_{\mu \nu}^{a}
$$

Notably, the linear term is absent. This is not surprising, since eq. (6.24) exhibits a global $Z_{2}$ symmetry under which $\digamma \rightarrow-\digamma$ and $\bar{Q}_{1} Q_{2} \rightarrow-\bar{Q}_{1} Q_{2}$. Had we included some 


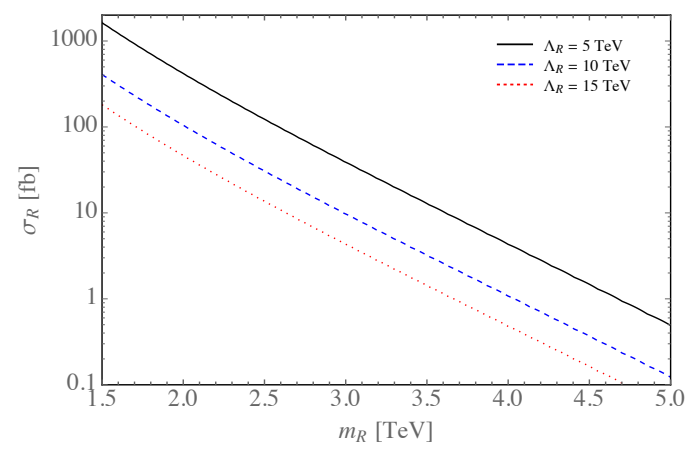

Figure 20. Single production cross section for a heavy resonance $R$ for a different values of the effective operator scale $\Lambda_{R}$, as defined in eq. (6.26). For reasonable values of $\Lambda_{R}$, if the branching ratio $\mathrm{BR}(R \rightarrow \digamma \digamma)$ is not too small, then $R$ could lead to significant contributions to the $\digamma$ pair production rate.

couplings which break the symmetry, such as $\kappa_{1} \digamma \bar{Q}_{1} Q_{1}, \kappa_{2} \digamma \bar{Q}_{2} Q_{2}$, then a linear term could be generated proportional to $\kappa_{1,2}$. As these terms break a symmetry they may be naturally small. In this way, in more involved models for the $\digamma$ excess, it may be that pair production is larger than expected based on a naïve extrapolation of the single production rate.

\subsection{Resonant pair production}

Finally, a scenario in which pair production may be considerably enhanced is given by a heavy resonance $R$ which is produced and subsequently decays to pairs of $\digamma, p p \rightarrow$ $R \rightarrow \digamma \digamma$. This possibility was discussed briefly in [7]. There are many different model possibilities, thus for simplicity we will only consider $R$ coupled to gluons and $\digamma$ as

$$
\mathcal{L}_{R} \supset \frac{g_{3}^{2}}{2 \Lambda_{R}} R G_{\mu \nu}^{2}+\frac{1}{2} A_{R \digamma \digamma} R \digamma^{2} .
$$

In figure 20 we show the single $R$ production rate as a function of the resonance mass and the effective scale $\Lambda_{R}$. This simple analysis demonstrates that, if the branching ratio $\mathrm{BR}(R \rightarrow \digamma \digamma)$ is not too small, $\digamma$ pair production may be significantly enhanced in the presence of new heavy resonances.

Pair production is also implied if $\digamma$ has SM gauge interactions (see e.g. [156]).

\subsection{Pair production phenomenology}

Although we will not attempt a thorough collider analyses of the pair production signature, it is useful to consider the typical character of pair production events. In figure 21 we show the invariant mass distribution of the resonance pairs and the $p_{T}$ spectrum of each resonance when produced from gluon fusion for the LET result as well as for two benchmark masses for heavy vector-like quarks. Although the location of the peak of these distributions lies in approximately the same place, regardless of the mass of the vector-like fermions in the loop, we see that further details such as the height of the peak and the tails of the distributions can vary significantly depending on the mass of the fermions in the loop. Thus, if we may be fortunate enough to see pair production in the next few years at the 

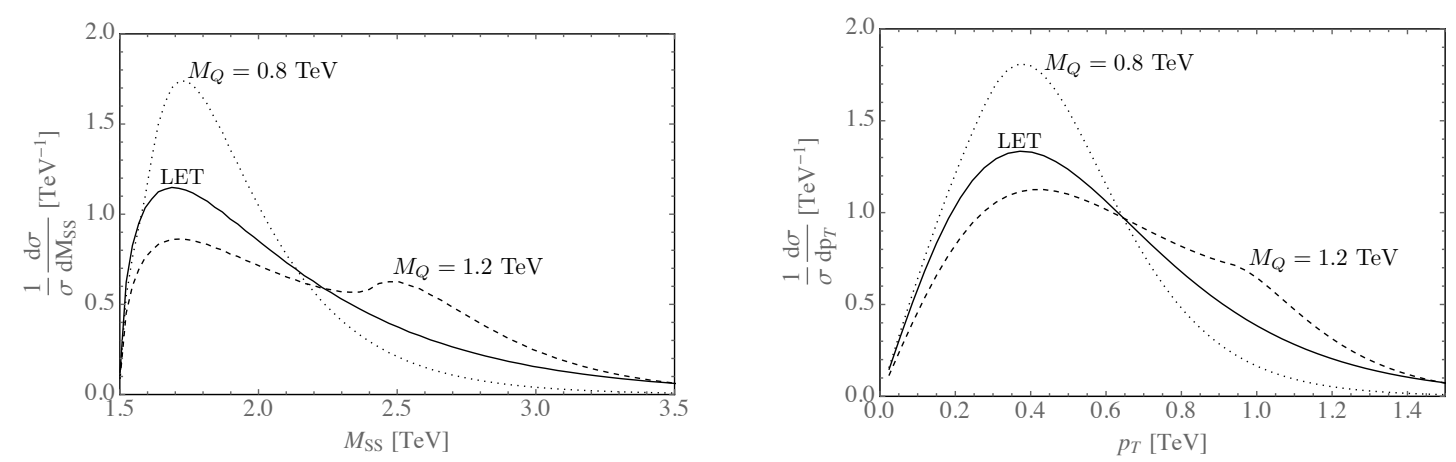

Figure 21. Normalised invariant mass and $p_{T}$ spectra for pairs of resonances produced from gluon fusion. Spectra are calculated using the LET result (solid black), and for two benchmarks, $M_{Q}=$ $800 \mathrm{GeV}$ (dotted) and $M_{Q}=1200 \mathrm{GeV}$ (dashed). A vanishing self-coupling is assumed. The invariant mass spectrum is peaked around $200 \mathrm{GeV}$ above the pair production threshold. The $p_{T}$ spectrum is peaked near to $400 \mathrm{GeV}$, indicating that in pair production events this typical boost should be expected for the diphoton or digluon system which may be useful for additional background reduction.

LHC, then with additional events it may be possible to gain some additional information on the nature of the coloured particles in the loop. However, by the stage that pair production of a $750 \mathrm{GeV}$ resonance had been observed one would expect any additional coloured particle to be observed directly, thus from this context pair production would provide a complementary probe of the coloured particles.

So far we have considered only production cross sections and distributions. In order to observe pair production it is necessary to be able to discriminate the final state over any SM background. The $4 j$ final state, already explored at $\sqrt{s}=8 \mathrm{TeV}$ in the search [141], has a good chance to probe the region of models parameters space where double production is enhanced. In fact we find QCD jet production $d \sigma / d m_{j j} \sim 200 \mathrm{fb} / \mathrm{GeV}$ in the region of phase-space where $m_{j j} \simeq 750 \mathrm{GeV}$ for candidate resonances mass $m_{j j}$ defined as in [157]. This means a background of about $40 \mathrm{pb}$ in a mass window $\left|m_{j j}-m_{\digamma}\right|<100 \mathrm{GeV}$.

Also the $j j \gamma \gamma$ final state, presently not investigated by the experiments, has very good chances to constrain the models. In particular the latter might have very low background rate and, in view of eq. (6.7), we estimate that a search in this channel at $13 \mathrm{TeV}$ would yield a useful bound in interesting regions of parameters space already with present luminosity. For this process we find an irreducible background from $p p \rightarrow j j \gamma \gamma$ around $0.2 \mathrm{fb}$ for $\left|m_{\gamma \gamma}-m_{\digamma}\right|<50 \mathrm{GeV}$ and $\left|m_{j j}-m_{\digamma}\right|<100 \mathrm{GeV}$. Given that the SM background is dominated by $q \rightarrow \gamma$ radiation, we implemented the following cuts: $\Delta R>0.4$ for any final state partons pair; $\left|\eta_{j}\right|<2.5,\left|\eta_{\gamma}\right|<2.37 ; p_{T, j}>150 \mathrm{GeV}, p_{T, \gamma_{1}}>40 \mathrm{GeV}, p_{T, \gamma_{2}}>30 \mathrm{GeV}$.

Finally, we remark that the $4 \gamma$ final state is interesting, as it is prominent in scenarios in which the production from electroweak boson initial states is not negligible. Signals in this final state can reach and exceed fraction of fb, while the irreducible background is negligible for projected luminosity of the LHC.

If other decay modes exist then additional signatures are possible. For example, if $\digamma$ can decay to dark matter then a search for $\gamma \gamma \mathbb{E}_{T}$ or $j j \mathbb{E}_{T}$ may reveal evidence for pair production. Once again the visible final state particles should reconstruct the resonant 
mass. In any case the kinematic characteristics described in this section may be used to aid the observation of pair production.

\section{Summary}

In this paper we have discussed a variety of observables that can be used to learn more about the nature of the digamma resonance $\digamma$ and extract its properties. Methods to discriminate a spin-0 from spin-2 diphoton resonance are already well established. In fact, the ATLAS and CMS collaborations already present analyses for both cases. Thus, from a phenomenological perspective, there is little to add on the topic of spin discrimination. For this reason we have focussed on the spin-0 hypothesis and on different open phenomenological questions. While our presentation was organised in terms of physical processes, in this section we summarise our results in terms of what can be learned from the different measurements and how these can be used to infer the properties of $\digamma$.

Our basic assumption is that $\digamma$ is a scalar particle and our conclusions are based on an Effective Field Theory (EFT) approach. Preliminary LHC data suggest that the neutral particle $\digamma$ must have a rather large coupling to photons, corresponding to an effective scale $\Lambda / M_{\digamma} \lesssim(10-20) c_{\gamma \gamma}$. Taking into account that $c_{\gamma \gamma}$ is induced at loop level, at least in a weakly-coupled theories the constraint on $\Lambda / M_{\digamma}$ restricts the applicability of the EFT theory and points towards the expectation that $\digamma$ is not an isolated particle, but part of a new-physics sector in the TeV domain. Whenever the EFT expansion breaks down, one must necessarily turn to a model-by-model analysis. Nevertheless, we believe that our EFT description can catch the main features of the underlying theory and that the observables discussed here are likely to bear fruit also in the context of complete models.

\subsection{Identifying the weak representation}

In this paper we have focused primarily on the case in which $\digamma$ is an electroweak singlet, but an important issue is to establish empirically if it is a singlet, a doublet with hypercharge $1 / 2$, or belongs to some other higher representation of $\mathrm{SU}(2)_{L} \otimes \mathrm{U}(1)_{Y}$ containing a neutral component. This can be tested as follows.

1. Higher representations will contain other components, some of which will be electrically charged, with mass around $750 \mathrm{GeV}$ and small mass splittings induced by electroweak breaking effects. Their single production is model dependent and can affect the profile of the bump at $750 \mathrm{GeV}$ in a measurable way. Pair production, via DrellYan processes, is universal and depends only on the electroweak quantum numbers.

2. Identifying the initial state of the production process will give indirect hints about the electroweak nature of $\digamma$. While a singlet can couple at dimension 5 to all SM particles, a doublet is more likely to be produced from quarks, to which it may have renormalisable couplings, than gluons or electroweak gauge bosons, to which can couple only through dimension- 6 operators. However, this conclusion is model dependent.

3. The different dimensionalities of the EFT couplings to quarks (dimension 5 for singlet $\digamma$ and 4 for doublet) and gauge bosons (dimension 5 for singlet and 6 for 
doublet) imply different $p_{T}$ spectra in $\digamma$ associated production. Although the applicability of the EFT is limited for the calculation of $p_{T}$ distributions, the EFT can describe the onset of the different behaviours of singlet and doublet $\digamma$. These results must then be compared with specific models.

Hereafter we will assume that $\digamma$ is an electroweak singlet.

\subsection{Identifying the initial state}

The identification of the parton process that produces $\digamma$ can be done using the following considerations.

1. Employing the dependence of parton luminosities on $\sqrt{s}$, one can use the values of $\sigma(p p \rightarrow \digamma)$ at different energies as discriminators of the initial process. In particular, the ratio between $\sigma(p p \rightarrow \digamma)$ at $13 \mathrm{TeV}$ versus $8 \mathrm{TeV}$, as summarised in section 2.1, is already favouring production from $g g, b \bar{b}, c \bar{c}$, and $s \bar{s}$ with respect to production from light quarks or photons.

2. Measuring decay channels other than $\digamma \rightarrow \gamma \gamma$ will give information on possible production mechanisms, since any initial state of the production process contributes to $\digamma$ decays. Di-jet measurements already constrain some parameter space for gluon production [7]. Other decay modes are illustrated in figure 2.

3. The rapidity distribution and the transverse momentum spectrum of the diphoton system retain features of the initial parton state and can be used to discriminate between light-quark and gluon or heavy-quark initiated productions [41].

4. The rate of $\digamma+$ jet production is a useful discriminator of the initial state. The ratios $\sigma_{\digamma j} / \sigma_{\digamma}$, with the same acceptance cuts applied across all production channels, are shown in table 1 . This ratio is $\mathcal{O}(27 \%)$ for gluon initiated production and $\mathcal{O}(6-9 \%)$ for heavy and light quark initial states.

5. For the $b$-quark initial state the ratio $\sigma_{\digamma b} / \sigma_{\digamma}$ is $6.2 \%$, whereas for all other initial states it is less than $1 \%$, see table 1 . Thus, $\digamma b$ associated production is an excellent indicator of $b$-quark initial states [41].

6. $\digamma$ production in association with a gauge or Higgs boson is a useful discriminator, see table 1 . In particular, no vector bosons accompanying $\digamma$ are expected from gluon initial states, and no $W$ from $b$ initial states. Taking ratios of $\digamma W, \digamma Z$, and $\digamma h$ provides us with additional handles to identify the production subprocess.

7. If $\digamma$ is a singlet produced from quarks, the particular structure of the operator $\digamma \bar{q} q H$ implies a sizeable three-body decay width, $\Gamma(\digamma \rightarrow q \bar{q} H) \sim 1 \% \times \Gamma(\digamma \rightarrow q \bar{q})$ where $H=\left\{h, Z, W^{ \pm}\right\}$(see section 3.2). 


\subsection{Measuring $\digamma$ couplings}

$\digamma$ couplings are crucial ingredients needed to understand the particle's nature.

1. $\digamma$ couplings can be extracted from production rates, as functions of the total width. If $\digamma$ is sufficiently broad that its width can be measured, than absolute determinations of its couplings are possible.

2. Furthermore, if the $\digamma$ resonance will turn out to be broad, by measuring its shape one could observe interference with the SM background $q \bar{q} \rightarrow \gamma \gamma$ amplitude in a way which, in principle, allows us to probe the structure of $\digamma$ couplings to quarks.

3. In an EFT approach valid up to operators with dimension 7 or higher, the 4 decay channels $\digamma \rightarrow \gamma \gamma, Z \gamma, Z Z, W W$ are described by 3 parameters (up to a discrete ambiguity) for a CP-even $\digamma$. This allows for one consistency condition that can be tested experimentally. For a CP-odd $\digamma$, one needs only 2 parameters (up to a discrete ambiguity) and 2 consistency conditions are obtained. The results are shown in figure 3 and figure 4.

4. The $\digamma$ invisible width can be derived by tagging $\digamma$ production with an extra jet.

5. Associated production processes $(\digamma+$ jet or $\digamma+V)$ can be used to probe interactions at different momenta, testing the derivative structure of effective couplings. Moreover, they can test the substructure of the effective couplings, beyond the domain of the EFT. This is because the extra jet, gauge or Higgs boson can be attached to the internal particles generating the effective couplings.

6. Pair production gives direct information about the properties of the UV completion of the EFT and the couplings of $\digamma$ to the new sector. In the strong coupling regime, and in the presence of approximate symmetries, pair production becomes especially relevant.

\subsection{Identifying the CP parity}

We discussed ways of measuring the $\mathrm{CP}$ properties of $\digamma$, in the three possible cases: CP even, $\mathrm{CP}$ odd, or undefined $\mathrm{CP}$ in a theory with explicit CP violation (see also [96] for a recent thorough study aimed at addressing this question).

The following three measurements rely only on $\digamma \rightarrow \gamma \gamma$ decays, which are guaranteed if the discovery is confirmed, and whose rates can then be unambiguously and modelindependently predicted.

1. The $\digamma \rightarrow \gamma \gamma$ decay guarantees the existence of $\digamma \rightarrow \gamma^{*} \gamma^{*} \rightarrow \ell^{+} \ell^{-} \ell^{\prime+} \ell^{\prime-}$ with $\ell, \ell^{\prime}=$ $\{e, \mu\}$, with a rate about $10^{-3}$ smaller than the $\gamma \gamma$ rate. The distributions have been computed in section 3.2 and they allow us to disentangle the $c_{\gamma \gamma}$ and $\tilde{c}_{\gamma \gamma}$ contributions.

2. The $\digamma \rightarrow \gamma \gamma$ decay guarantees the existence of $\digamma \rightarrow \gamma \gamma \rightarrow e^{+} e^{-} e^{+} e^{-}$events, where the $\gamma$ convert to electron-positron pairs in the detector matter, giving access to the photon polarisations [158]. The small angle between each $e^{+} e^{-}$pair however makes this measurement very difficult. 
3. The $\digamma \rightarrow \gamma \gamma$ decay guarantees the existence of $p p \rightarrow j j \digamma$ events, where the jets are emitted at least from initial state partons. The rate is larger if $\digamma$ is produced via $g g$ collisions. As discussed in section 4.1, the angular distribution of the two jets is sensitive to the CP parity of $\digamma$.

The following measurements rely on $\digamma \rightarrow Z Z, Z \gamma$, one of which (at least) is always guaranteed, although at the moment we cannot tell how large the corresponding rates will be.

4. The parity of $\digamma$ can be measured from the distribution of $\digamma \rightarrow Z Z \rightarrow \ell^{+} \ell^{-} \ell^{\prime+} \ell^{\prime-}$ events, similarly to what is done for the Higgs.

5. The $\digamma \rightarrow Z \gamma$ decay allows for a measurement of the $\digamma$ parity using $Z \rightarrow \ell^{+} \ell^{-}$and provided that $\gamma$ converts into $\ell^{+} \ell^{-}$either virtually or in matter, as discussed above.

6. The $\digamma Z Z$ and $\digamma Z \gamma$ couplings imply a rate for $p p \rightarrow \digamma Z$ whose angular distribution allows for a reconstruction of the CP-parity of $\digamma$ as discussed in section 5.1.

Finally we have a possibility which is not guaranteed by the diphoton decay, but which would be completely decisive.

7. If $\digamma$ decays into $h h$, its observation would immediately imply that $\digamma$ is a CP-even scalar, provided that $\mathrm{CP}$ is conserved.

\subsection{Pair production}

In this paper we have emphasised pair production as a new tool for investigating the properties of $\digamma$. It is not possible to make definitive model-independent predictions for pair production. The reason is that while single production already gives some information on possible dimension 5 couplings of $\digamma$ to SM states, pair production involves not only the same dimension- 5 operators but also a priori uncorrelated dimension 6 operators and an $\digamma^{3}$ self interaction. Pair production cross sections are expressed in terms of a well-motived set of operators in section 6.1.

A particularly simple, but representative, class of models provides a correlation between operators in terms of model parameters, see eq. (6.9). Furthermore, the model assumptions combined with the required single production rate allow for the pair production rates to be predicted. In addition, one is no longer constrained to an EFT description and rates may also be calculated when the new states are light. In section 6.2 a popular model of $\digamma$ coupled to charged and coloured fermions is studied. In both the low-energy theorem approximation and in the full calculation employing the one loop form factors, a number of qualitative conclusions can be drawn. Constraints on the production of pairs of di-jet resonances are already relevant, excluding some of the parameter space. This suggests that in the future this final state may reveal evidence for the pair production of $\digamma$. The $\gamma \gamma g g$ final state is more challenging, with smaller cross sections. This is also true for the $4 \gamma$ final state. 


\section{Acknowledgments}

This work was supported by the ERC grant NEO-NAT. JFK acknowledges the financial support from the Slovenian Research Agency (research core funding No. P1-0035). The work of RT is supported by Swiss National Science Foundation under grants CRSII2160814 and 200020-150060. RT also acknowledges, for computing resources, Heidi, the computing support of INFN Padova, and the grant SNF Sinergia no. CRSII2-141847. We thank Clifford Cheung, José Ramon Espinosa, Stefano Frixione, Riccardo Rattazzi, Michele Redi, Javi Serra for useful discussions.

\section{A Effective Lagrangian in the unitary gauge}

The effective Lagrangian for the scalar singlet in eqs. (2.3), (2.4), (2.5), (2.6) and (2.9) can be expanded in the unitary gauge as follows:

$$
\begin{aligned}
V(\digamma, H)= & \frac{1}{2} m_{\digamma}^{2} \digamma^{2}+\kappa_{\digamma} m_{\digamma} \digamma^{3}+\lambda_{\digamma} \digamma^{4}+\kappa_{\digamma H} m_{\digamma} \digamma h(h / 2+v)+\lambda_{\digamma H} \digamma^{2} h(h / 2+v), \\
\mathscr{L}_{5}^{\text {even }}= & \frac{\digamma}{\Lambda}\left[c_{g g} \frac{g_{3}^{2}}{2} G_{\mu \nu}^{a} G^{a \mu \nu}+c_{W W} \frac{g_{2}^{2}}{2} W_{\mu \nu}^{+} W^{-\mu \nu}+c_{Z Z} \frac{e^{2}}{2} Z_{\mu \nu} Z^{\mu \nu}+c_{\gamma \gamma} \frac{e^{2}}{2} \gamma_{\mu \nu} \gamma^{\mu \nu}+c_{\gamma Z} \frac{e^{2}}{2} \gamma_{\mu \nu} Z^{\mu \nu}\right. \\
& +\frac{c_{\psi}}{\sqrt{2}}(h+v) \bar{\psi} \psi+\frac{c_{H} e^{2}}{8 c_{\mathrm{W}}^{2} s_{\mathrm{W}}^{2}}\left(h^{2}+2 h v+2 v^{2}\right)\left(2 c_{\mathrm{W}}^{2} W_{\mu} W_{\mu}^{\dagger}+Z_{\mu}^{2}\right)+\frac{c_{H}}{2} \partial_{\mu}(h)^{2} \\
& \left.-\frac{1}{4} c_{H}^{\prime} h\left(h^{3}+4 h^{2} v+6 h v^{2}+4 v^{3}\right)\right]+\frac{c_{\digamma 3}}{\Lambda} \frac{\digamma\left(\partial_{\mu} \digamma\right)^{2}}{2} \\
\mathscr{L}_{5}^{\text {odd }}= & \frac{\digamma}{\Lambda}\left[\tilde{c}_{g g} \frac{g_{3}^{2}}{2} G_{\mu \nu}^{a} \tilde{G}^{a \mu \nu}+\tilde{c}_{W W} \frac{g_{2}^{2}}{2} W_{\mu \nu}^{+} \tilde{W}^{-\mu \nu}+\tilde{c}_{Z Z} \frac{e^{2}}{2} Z_{\mu \nu} \tilde{Z}^{\mu \nu}+\tilde{c}_{\gamma \gamma} \frac{e^{2}}{2} \gamma_{\mu \nu} \tilde{\gamma}^{\mu \nu}+\right. \\
& \left.+\tilde{c}_{\gamma Z} \frac{e^{2}}{2} \gamma_{\mu \nu} \tilde{Z}^{\mu \nu}+i \frac{\tilde{c}_{\psi}}{\sqrt{2}}(h+v) \bar{\psi} \gamma^{5} \psi\right], \\
\mathscr{L}_{6}= & \frac{\digamma^{2}}{\Lambda^{2}}\left[c_{g g}^{(6)} \frac{g_{3}^{2}}{2} G_{\mu \nu}^{a} G^{a \mu \nu}+c_{W W}^{(6)} \frac{g_{2}^{2}}{2} W_{\mu \nu}^{+} W^{-\mu \nu}+c_{Z Z}^{(6)} \frac{e^{2}}{2} Z_{\mu \nu} Z^{\mu \nu}+c_{\gamma \gamma}^{(6)} \frac{e^{2}}{2} \gamma_{\mu \nu} \gamma^{\mu \nu}+c_{\gamma Z}^{(6)} \frac{e^{2}}{2} \gamma_{\mu \nu} Z^{\mu \nu}\right. \\
& +\frac{c_{\psi}^{(6)}}{\sqrt{2}}(h+v) \bar{\psi} \psi+\frac{c_{H}^{(6)} e^{2}}{8 c_{\mathrm{W}}^{2} s_{\mathrm{W}}^{2}}\left(h^{2}+2 h v+2 v^{2}\right)\left(2 c_{\mathrm{W}}^{2} W_{\mu} W_{\mu}^{\dagger}+Z_{\mu}^{2}\right)+\frac{c_{H}^{(6)}}{2} \partial_{\mu}(h)^{2} \\
& \left.-\frac{1}{4} c_{H}^{(6) \prime} h\left(h^{3}+4 h^{2} v+6 h v^{2}+4 v^{3}\right)\right]+\frac{c_{H 2}^{(6)}}{\Lambda^{2}} \frac{\left(\partial_{\mu} \digamma\right)^{2}}{2} h(h / 2+v)+\mathcal{O}\left(\digamma^{4}\right),
\end{aligned}
$$

\section{B $\digamma$ decay widths including the mixing with the Higgs}

In this section we write down the complete formulæ for the decay widths of $\digamma$ including the effect of its mixing with Higgs boson $h$. The operators proportional to $\kappa_{\digamma H}$ and $c_{H}^{\prime}$ induce, after EWSB, a mixing between $\digamma$ and the Higgs given by

$$
\mathscr{L}_{\text {mix }}=-\frac{1}{2} m_{H}^{2} h^{2}-\frac{1}{2} m_{\digamma}^{2} \digamma^{2}-h \digamma v\left(\kappa_{\digamma H} m_{\digamma}+\frac{c_{H}^{\prime} v^{2}}{\Lambda}\right),
$$

where $m_{H}$ is the Higgs mass parameter (not yet the physical Higgs mass $M_{h}$ ). The mass matrix is diagonalised by the rotation

$$
h \rightarrow h \cos \theta+\digamma \sin \theta, \quad \digamma \rightarrow \digamma \cos \theta-h \sin \theta,
$$


with mixing angle

$$
\tan 2 \theta=\frac{2 v\left(m_{\digamma} \kappa_{\digamma H}+c_{H}^{\prime} v^{2} / \Lambda\right)}{m_{\digamma}^{2}-m_{H}^{2}} .
$$

The masses of the physical $h$ and $\digamma$ eigenstates are

$$
\begin{aligned}
& M_{h}^{2}=\frac{1}{2}\left[m_{\digamma}^{2}+m_{H}^{2}-\sqrt{\left(m_{H}^{2}-m_{\digamma}^{2}\right)^{2}+4 v^{2}\left(\kappa_{\digamma H} m_{\digamma}+c_{H}^{\prime} v^{2} / \Lambda\right)^{2}}\right], \\
& M_{\digamma}^{2}=\frac{1}{2}\left[m_{\digamma}^{2}+m_{H}^{2}+\sqrt{\left(m_{H}^{2}-m_{\digamma}^{2}\right)^{2}+4 v^{2}\left(\kappa_{\digamma H} m_{\digamma}+c_{H}^{\prime} v^{2} / \Lambda\right)^{2}}\right],
\end{aligned}
$$

where we have used uppercase letters to indicate the physical masses. After diagonalising the mass mixing, all the couplings of $\digamma$ to SM particles acquire corrections of order $c_{H}^{\prime} v / \Lambda$. Moreover, after mixing with the Higgs, $\digamma$ inherits the Higgs couplings to SM particles, suppressed by $s_{\theta} \equiv \sin \theta$. In order to include these contributions in the $\digamma$ interactions and decay widths, we parametrise the loop-induced Higgs couplings to $g g, \gamma \gamma, \gamma Z$ as

$$
\mathscr{L}_{h-\text { loop }}=\frac{g_{3}^{2} c_{h, g}}{v} h G_{\mu \nu}^{2}+\frac{e^{2} c_{h, \gamma}}{v} h F_{\mu \nu}^{2}+\frac{e^{2} c_{h, \gamma Z}}{v s_{\mathrm{W}} c_{\mathrm{W}}} h F_{\mu \nu} Z^{\mu \nu} .
$$

The value of the $c$ coefficients in the SM (and its dependence on the loop function) can be found, for instance, in [159]. The complete expressions of the widths taking into account all the subleading corrections are given by

$$
\begin{aligned}
\Gamma(\digamma \rightarrow \gamma \gamma)= & \frac{\pi \alpha^{2} M_{\digamma}^{3}}{\Lambda^{2}}\left[c_{\gamma \gamma}^{2}\left(c_{\theta}+\frac{2 s_{\theta} c_{h, \gamma} \Lambda}{c_{\gamma \gamma}}\right)^{2}+\tilde{c}_{\gamma \gamma}^{2}\right], \\
\Gamma(\digamma \rightarrow g g)= & \frac{8 \pi \alpha_{3}^{2} M_{\digamma}^{3}}{\Lambda^{2}}\left[c_{g g}^{2}\left(c_{\theta}+2 \frac{s_{\theta} c_{h, g} \Lambda}{c_{g g} v}\right)^{2}+\tilde{c}_{g g}^{2}\right], \\
\Gamma(\digamma \rightarrow \psi \bar{\psi})= & \frac{N_{\psi} M_{\digamma} v^{2}}{16 \pi \Lambda^{2}}\left[\left(c_{\psi} c_{\theta}-y_{\psi} s_{\theta}\right)^{2} f_{\psi}\left(x_{\psi}\right)+\tilde{c}_{\psi}^{2} \tilde{f}_{\psi}\left(x_{\psi}\right)\right], \\
\Gamma(\digamma \rightarrow \gamma Z)= & \frac{2 \pi \alpha^{2} M_{\digamma}^{3}}{s_{\mathrm{W}}^{2} c_{\mathrm{W}}^{2} \Lambda^{2}}\left[c_{\gamma Z}^{2}\left(c_{\theta}-s_{\mathrm{W}} c_{\mathrm{W}} s_{\theta} \frac{c_{h, \gamma Z} \Lambda}{c_{\gamma Z} v}\right)^{2}+\tilde{c}_{\gamma Z}^{2}\right] f_{\gamma Z}\left(x_{Z}\right), \\
\Gamma(\digamma \rightarrow Z Z)= & \frac{\pi \alpha^{2} M_{\digamma}^{3}}{s_{\mathrm{W}}^{4} c_{\mathrm{W}}^{4} \Lambda^{2}}\left[c_{\theta}^{2} c_{Z Z}^{2} f^{(T T)}\left(x_{Z}\right)+\tilde{c}_{Z Z}^{2} \tilde{f}^{(T T)}\left(x_{Z}\right)-\frac{3 v c_{\theta} c_{H} c_{Z Z}\left(v c_{\theta}+2 \Lambda s_{\theta} / c_{H}\right)}{4 m_{\digamma}^{2}} f^{(L T)}\left(x_{Z}\right)\right. \\
& \left.+\frac{v^{2} c_{H}^{2}\left(v c_{\theta}+2 \Lambda s_{\theta} / c_{H}\right)^{2}}{128 M_{Z}^{4}} f^{(L L)}\left(x_{Z}\right)\right], \\
\Gamma(\digamma \rightarrow W W)= & \frac{2 \pi \alpha^{2} M_{\digamma}^{3}}{s_{\mathrm{W}}^{4} \Lambda^{2}}\left[c_{\theta}^{2} c_{W W}^{2} f^{(T T)}\left(x_{W}\right)+\tilde{c}_{W W}^{2} \tilde{f}^{(T T)}\left(x_{W}\right)-\frac{3 v c_{\theta} c_{H} c_{W W}\left(v c_{\theta}+2 \Lambda s_{\theta} / c_{H}\right)}{4 m_{\digamma}^{2}} f^{(L T)}\left(x_{W}\right)\right. \\
& \left.+\frac{v^{2} c_{H}^{2}\left(v c_{\theta}+2 \Lambda s_{\theta} / c_{H}\right)^{2}}{128 M_{W}^{4}} f^{(L L)}\left(x_{W}\right)\right], \\
\Gamma(\digamma \rightarrow h h)= & \frac{M_{\digamma}^{3}}{128 \pi \Lambda^{4} v^{2}\left(M_{\digamma}^{2}-2 M_{H}^{2}\right)^{2}}\left[2 v^{4} s_{\theta}^{3} c_{H}^{(6) \prime 2}-4 v^{4} c_{\theta}^{2} s_{\theta} c_{H}^{(6) \prime 2}-12 \Lambda v^{3} c_{\theta} s_{\theta}^{2} c_{H}^{\prime}\right. \\
& +6 \Lambda v^{3} c_{\theta}^{3} c_{H}^{\prime}+v^{2} c_{H}^{(6) 2} s_{\theta}\left(M_{\digamma}^{2}\left(s_{\theta}^{2}-2 c_{\theta}^{2}\right)-2 M_{H}^{2} s_{\theta}^{2}\right)+\Lambda v c_{\theta} c_{H}\left(M_{\digamma}^{2}\left(c_{\theta}^{2}-2 s_{\theta}^{2}\right)-2 c_{\theta}^{2} M_{H}^{2}\right) \\
& +6 \Lambda^{2} c_{\theta}^{2} M_{H}^{2} s_{\theta}-2 \Lambda v c_{\theta} c_{\digamma} M_{H}^{2} s_{\theta}^{2}+3 \Lambda M_{\digamma}^{2} v c_{\theta} c_{\digamma} s_{\theta}^{2}+12 \Lambda^{2} M_{\digamma} v c_{\theta} s_{\theta}^{2} \kappa_{\digamma} \\
& \left.-4 \Lambda^{2} M_{\digamma} v c_{\theta} s_{\theta}^{2} \kappa_{\digamma}+2 \Lambda^{2} M_{\digamma} v c_{\theta}^{3} \kappa_{\digamma H}-8 \Lambda^{2} v^{2} c_{\theta}^{2} s_{\theta} \lambda_{\digamma H}+4 \Lambda^{2} v^{2} s_{\theta}^{3} \lambda_{\digamma}\right]^{2} f_{h}\left(x_{h}\right), \quad(\mathrm{B} .6 \mathrm{~g})
\end{aligned}
$$

where $x_{P}=M_{P}^{2} / M_{\digamma}^{2}$ and the phase space functions $f$ and $\tilde{f}$ are given by

$$
f_{\psi}(x)=(1-4 x)^{3 / 2},
$$




$$
\begin{aligned}
\tilde{f}_{\psi}(x) & =\sqrt{1-4 x}, \\
f_{\gamma Z}(x) & =(1-x)^{3}, \\
f^{(T T)}(x) & =\left(1-4 x+6 x^{2}\right) \sqrt{1-4 x}, \\
\tilde{f}^{(T T)}(x) & =(1-4 x)^{3 / 2}, \\
f^{(L L)}(x) & =\left(1-4 x+12 x^{2}\right) \sqrt{1-4 x}, \\
f^{(L T)}(x) & =(1-2 x) \sqrt{1-4 x}, \\
f_{h}(x) & =(1-2 x)^{2} \sqrt{1-4 x} .
\end{aligned}
$$

Open Access. This article is distributed under the terms of the Creative Commons Attribution License (CC-BY 4.0), which permits any use, distribution and reproduction in any medium, provided the original author(s) and source are credited.

\section{References}

[1] ATLAS collaboration, Search for resonances decaying to photon pairs in $3.2 \mathrm{fb}^{-1}$ of $p p$ collisions at $\sqrt{s}=13 \mathrm{TeV}$ with the ATLAS detector, ATLAS-CONF-2015-081, CERN, Geneva Switzerland (2015).

[2] CMS collaboration, Search for new physics in high mass diphoton events in proton-proton collisions at $\sqrt{s}=13 \mathrm{TeV}$, CMS-PAS-EXO-15-004, CERN, Geneva Switzerland (2015).

[3] ATLAS collaboration, M. Delmastro, Diphoton searches in ATLAS, talk at the Moriond 2016 conference, https://indico.in2p3.fr/e/moriondEW2016, La Thuile Italy (2016).

[4] CMS collaboration, P. Musella, Diphoton searches in CMS, talk at the Moriond 2016 conference, https://indico.in2p3.fr/e/moriondEW2016, La Thuile Italy (2016).

[5] ATLAS collaboration, Search for resonances in diphoton events with the ATLAS detector at $\sqrt{s}=13 \mathrm{TeV}$, ATLAS-CONF-2016-018, CERN, Geneva Switzerland (2016).

[6] CMS collaboration, Search for new physics in high mass diphoton events in $3.3 \mathrm{fb}^{-1}$ of proton-proton collisions at $\sqrt{s}=13 \mathrm{TeV}$ and combined interpretation of searches at $8 \mathrm{TeV}$ and $13 \mathrm{TeV}$, CMS-PAS-EXO-16-018, CERN, Geneva Switzerland (2016).

[7] R. Franceschini et al., What is the $\gamma \gamma$ resonance at 750 GeV?, JHEP 03 (2016) 144 [arXiv: 1512.04933] [INSPIRE].

[8] J. Ellis, S.A.R. Ellis, J. Quevillon, V. Sanz and T. You, On the interpretation of a possible $\sim 750 \mathrm{GeV}$ particle decaying into $\gamma \gamma$, JHEP 03 (2016) 176 [arXiv: 1512.05327] [INSPIRE].

[9] A. Falkowski, O. Slone and T. Volansky, Phenomenology of a $750 \mathrm{GeV}$ singlet, JHEP 02 (2016) 152 [arXiv: 1512.05777] [INSPIRE].

[10] R.S. Gupta, S. Jäger, Y. Kats, G. Perez and E. Stamou, Interpreting a $750 \mathrm{GeV}$ diphoton resonance, arXiv:1512.05332 [INSPIRE].

[11] P. Agrawal, J. Fan, B. Heidenreich, M. Reece and M. Strassler, Experimental considerations motivated by the diphoton excess at the LHC, JHEP 06 (2016) 082 [arXiv:1512.05775] [INSPIRE].

[12] J.S. Kim, K. Rolbiecki and R. Ruiz de Austri, Model-independent combination of diphoton constraints at $750 \mathrm{GeV}$, Eur. Phys. J. C 76 (2016) 251 [arXiv:1512.06797] [INSPIRE]. 
[13] S. Di Chiara, L. Marzola and M. Raidal, First interpretation of the $750 \mathrm{GeV}$ diphoton resonance at the LHC, Phys. Rev. D 93 (2016) 095018 [arXiv: 1512.04939] [InSPIRE].

[14] S. Jung, J. Song and Y.W. Yoon, How resonance-continuum interference changes $750 \mathrm{GeV}$ diphoton excess: signal enhancement and peak shift, JHEP 05 (2016) 009 [arXiv: 1601.00006] [INSPIRE].

[15] F. D'Eramo, J. de Vries and P. Panci, A $750 \mathrm{GeV}$ portal: LHC phenomenology and dark matter candidates, JHEP 05 (2016) 089 [arXiv:1601.01571] [INSPIRE].

[16] M. Fabbrichesi and A. Urbano, The breaking of the $\mathrm{SU}(2)_{L} \times \mathrm{U}(1)_{Y}$ symmetry: the $750 \mathrm{GeV}$ resonance at the $\mathrm{LHC}$ and perturbative unitarity, arXiv: 1601.02447 [INSPIRE].

[17] J. Cao, L. Shang, W. Su, Y. Zhang and J. Zhu, Interpreting the $750 \mathrm{GeV}$ diphoton excess in the minimal dilaton model, Eur. Phys. J. C 76 (2016) 239 [arXiv:1601.02570] [InSPIRE].

[18] A. Djouadi, J. Ellis, R. Godbole and J. Quevillon, Future collider signatures of the possible $750 \mathrm{GeV}$ state, JHEP 03 (2016) 205 [arXiv: 1601.03696] [inSPIRE].

[19] M.R. Buckley, Wide or narrow? The phenomenology of $750 \mathrm{GeV}$ diphotons, Eur. Phys. J. C 76 (2016) 345 [arXiv: 1601.04751] [INSPIRE].

[20] J. Bernon, A. Goudelis, S. Kraml, K. Mawatari and D. Sengupta, Characterising the $750 \mathrm{GeV}$ diphoton excess, JHEP 05 (2016) 128 [arXiv: 1603.03421] [INSPIRE].

[21] G. Panico, L. Vecchi and A. Wulzer, Resonant diphoton phenomenology simplified, JHEP 06 (2016) 184 [arXiv: 1603.04248] [INSPIRE].

[22] J.F. Kamenik, B.R. Safdi, Y. Soreq and J. Zupan, Comments on the diphoton excess: critical reappraisal of effective field theory interpretations, JHEP 07 (2016) 042 [arXiv: 1603.06566] [INSPIRE].

[23] G. Cynolter, J. . Kovács and E. Lendvai, Diphoton excess and VV-scattering, Mod. Phys. Lett. A 31 (2016) 1650133 [arXiv:1604.01008] [INSPIRE].

[24] W. Chao, R. Huo and J.-H. Yu, The minimal scalar-stealth top interpretation of the diphoton excess, arXiv: 1512.05738 [INSPIRE].

[25] S. Knapen, T. Melia, M. Papucci and K. Zurek, Rays of light from the LHC, Phys. Rev. D 93 (2016) 075020 [arXiv: 1512.04928] [InSPIRE].

[26] B. Dutta, Y. Gao, T. Ghosh, I. Gogoladze and T. Li, Interpretation of the diphoton excess at CMS and ATLAS, Phys. Rev. D 93 (2016) 055032 [arXiv: 1512.05439] [INSPIRE].

[27] S.D. McDermott, P. Meade and H. Ramani, Singlet scalar resonances and the diphoton excess, Phys. Lett. B 755 (2016) 353 [arXiv:1512.05326] [INSPIRE].

[28] A. Kobakhidze, F. Wang, L. Wu, J.M. Yang and M. Zhang, 750 GeV diphoton resonance in a top and bottom seesaw model, Phys. Lett. B 757 (2016) 92 [arXiv:1512.05585] [INSPIRE].

[29] P. Cox, A.D. Medina, T.S. Ray and A. Spray, Diphoton excess at $750 \mathrm{GeV}$ from a radion in the bulk-Higgs scenario, arXiv:1512.05618 [INSPIRE].

[30] A. Ahmed, B.M. Dillon, B. Grzadkowski, J.F. Gunion and Y. Jiang, Higgs-radion interpretation of $750 \mathrm{GeV}$ di-photon excess at the LHC, arXiv:1512.05771 [INSPIRE].

[31] E. Megias, O. Pujolàs and M. Quirós, On dilatons and the LHC diphoton excess, JHEP 05 (2016) 137 [arXiv: 1512.06106] [INSPIRE].

[32] S. Ghosh, A. Kundu and S. Ray, Potential of a singlet scalar enhanced Standard Model, Phys. Rev. D 93 (2016) 115034 [arXiv:1512.05786] [inSPIRE]. 
[33] L. Berthier, J.M. Cline, W. Shepherd and M. Trott, Effective interpretations of a diphoton excess, JHEP 04 (2016) 084 [arXiv: 1512.06799] [InSPIRE].

[34] J. Cao, C. Han, L. Shang, W. Su, J.M. Yang and Y. Zhang, Interpreting the $750 \mathrm{GeV}$ diphoton excess by the singlet extension of the Manohar-Wise model, Phys. Lett. B 755 (2016) 456 [arXiv: 1512.06728] [INSPIRE].

[35] I. Chakraborty and A. Kundu, Diphoton excess at $750 \mathrm{GeV}$ : singlet scalars confront triviality, Phys. Rev. D 93 (2016) 055003 [arXiv: 1512.06508] [INSPIRE].

[36] H. Han, S. Wang and S. Zheng, Scalar explanation of diphoton excess at LHC, Nucl. Phys. B 907 (2016) 180 [arXiv: 1512.06562] [INSPIRE].

[37] S. Chang, A simple U(1) gauge theory explanation of the diphoton excess, Phys. Rev. D 93 (2016) 055016 [arXiv: 1512.06426] [INSPIRE].

[38] D. Buttazzo, A. Greljo and D. Marzocca, Knocking on new physics' door with a scalar resonance, Eur. Phys. J. C 76 (2016) 116 [arXiv:1512.04929] [INSPIRE].

[39] C.W. Murphy, Vector leptoquarks and the $750 \mathrm{GeV}$ diphoton resonance at the LHC, Phys. Lett. B 757 (2016) 192 [arXiv:1512.06976] [INSPIRE].

[40] F. Goertz, J.F. Kamenik, A. Katz and M. Nardecchia, Indirect constraints on the scalar di-photon resonance at the LHC, JHEP 05 (2016) 187 [arXiv: 1512.08500] [INSPIRE].

[41] J. Gao, H. Zhang and H.X. Zhu, Diphoton excess at $750 \mathrm{GeV}$ : gluon-gluon fusion or quark-antiquark annihilation?, Eur. Phys. J. C 76 (2016) 348 [arXiv:1512.08478] [INSPIRE].

[42] S. Chakraborty, A. Chakraborty and S. Raychaudhuri, Diphoton resonance at $750 \mathrm{GeV}$ in the broken MRSSM, arXiv:1512.07527 [INSPIRE].

[43] K. Cheung, P. Ko, J.S. Lee, J. Park and P.-Y. Tseng, A Higgcision study on the $750 \mathrm{GeV}$ di-photon resonance and $125 \mathrm{GeV}$ SM Higgs boson with the Higgs-singlet mixing, arXiv: 1512.07853 [INSPIRE].

[44] C. Cai, Z.-H. Yu and H.-H. Zhang, $750 \mathrm{GeV}$ diphoton resonance as a singlet scalar in an extra dimensional model, Phys. Rev. D 93 (2016) 075033 [arXiv:1512.08440] [INSPIRE].

[45] G. Li, Y.-N. Mao, Y.-L. Tang, C. Zhang, Y. Zhou and S.-H. Zhu, Pseudoscalar decaying only via loops as an explanation for the $750 \mathrm{GeV}$ diphoton excess, Phys. Rev. Lett. 116 (2016) 151803 [arXiv:1512.08255] [INSPIRE].

[46] Y.-L. Tang and S.-H. Zhu, NMSSM extended with vector-like particles and the diphoton excess on the $L H C$, arXiv:1512.08323 [INSPIRE].

[47] Q.-H. Cao, Y. Liu, K.-P. Xie, B. Yan and D.-M. Zhang, Diphoton excess, low energy theorem and the 331 model, Phys. Rev. D 93 (2016) 075030 [arXiv:1512.08441] [InSPIRE].

[48] D. Chway, R. Dermíšek, T.H. Jung and H.D. Kim, Glue to light signal of a new particle, arXiv: 1512.08221 [INSPIRE].

[49] I. Low and J. Lykken, Implications of gauge invariance on a heavy diphoton resonance, arXiv: 1512.09089 [INSPIRE].

[50] B. Dutta et al., Diphoton excess in consistent supersymmetric $\mathrm{SU}(5)$ models with vector-like particles, arXiv: 1601.00866 [INSPIRE].

[51] J. Gu and Z. Liu, Physics implications of the diphoton excess from the perspective of renormalization group flow, Phys. Rev. D 93 (2016) 075006 [arXiv: 1512.07624] [INSPIRE]. 
[52] S. Kanemura, N. Machida, S. Odori and T. Shindou, Diphoton excess at $750 \mathrm{GeV}$ in an extended scalar sector, arXiv: 1512.09053 [INSPIRE].

[53] S. Gopalakrishna, T.S. Mukherjee and S. Sadhukhan, Extra neutral scalars with vectorlike fermions at the LHC, Phys. Rev. D 93 (2016) 055004 [arXiv: 1504.01074] [InSPIRE].

[54] S. Abel and V.V. Khoze, Photo-production of a $750 \mathrm{GeV}$ di-photon resonance mediated by Kaluza-Klein leptons in the loop, JHEP 05 (2016) 063 [arXiv:1601.07167] [INSPIRE].

[55] J. Kawamura and Y. Omura, Diphoton excess at $750 \mathrm{GeV}$ and LHC constraints in models with vectorlike particles, Phys. Rev. D 93 (2016) 115011 [arXiv:1601.07396] [INSPIRE].

[56] M.J. Dolan, J.L. Hewett, M. Krämer and T.G. Rizzo, Simplified models for Higgs physics: singlet scalar and vector-like quark phenomenology, JHEP 07 (2016) 039 [arXiv: 1601.07208] [INSPIRE].

[57] D. Aloni, K. Blum, A. Dery, A. Efrati and Y. Nir, On a possible large width $750 \mathrm{GeV}$ diphoton resonance at ATLAS and CMS, arXiv:1512.05778 [INSPIRE].

[58] A. Salvio, F. Staub, A. Strumia and A. Urbano, On the maximal diphoton width, JHEP 03 (2016) 214 [arXiv: 1602.01460] [InSPIRE].

[59] A. Alves, A.G. Dias and K. Sinha, The $750 \mathrm{GeV}$ S-cion: where else should we look for it?, Phys. Lett. B 757 (2016) 39 [arXiv: 1512.06091] [InSPIRE].

[60] H. Ito, T. Moroi and Y. Takaesu, Studying $750 \mathrm{GeV}$ di-photon resonance at photon-photon collider, Phys. Lett. B 756 (2016) 147 [arXiv:1601.01144] [INSPIRE].

[61] M. He, X.-G. He and Y. Tang, A $\gamma \gamma$ collider for the $750 \mathrm{GeV}$ resonant state, Phys. Lett. B 759 (2016) 166 [arXiv:1603.00287] [INSPIRE].

[62] C. Gross, O. Lebedev and J.M. No, Drell-Yan constraints on new electroweak states and the di-photon anomaly, arXiv: 1602.03877 [INSPIRE].

[63] K.J. Bae, M. Endo, K. Hamaguchi and T. Moroi, Diphoton excess and running couplings, Phys. Lett. B 757 (2016) 493 [arXiv: 1602.03653] [INSPIRE].

[64] Y. Hamada, H. Kawai, K. Kawana and K. Tsumura, Models of the LHC diphoton excesses valid up to the Planck scale, Phys. Rev. D 94 (2016) 014007 [arXiv:1602.04170] [INSPIRE].

[65] J. Chakrabortty, A. Choudhury, P. Ghosh, S. Mondal and T. Srivastava, Di-photon resonance around $750 \mathrm{GeV}$ : shedding light on the theory underneath, arXiv:1512.05767 [INSPIRE].

[66] F. Staub et al., Precision tools and models to narrow in on the $750 \mathrm{GeV}$ diphoton resonance, arXiv: 1602.05581 [INSPIRE].

[67] L.M. Carpenter, R. Colburn and J. Goodman, Supersoft SUSY models and the $750 \mathrm{GeV}$ diphoton excess, beyond effective operators, Phys. Rev. D 94 (2016) 015016 [arXiv: 1512.06107] [INSPIRE].

[68] C. Csáki and L. Randall, A diphoton resonance from bulk RS, JHEP 07 (2016) 061 [arXiv: 1603.07303] [INSPIRE].

[69] N. Liu, W. Wang, M. Zhang and R. Zheng, $750 \mathrm{GeV}$ diphoton resonance in a vector-like extension of Hill model, arXiv:1604.00728 [INSPIRE].

[70] Q.-H. Cao, Y. Liu, K.-P. Xie, B. Yan and D.-M. Zhang, A boost test of anomalous diphoton resonance at the $L H C$, arXiv: 1512.05542 [INSPIRE]. 
[71] W. Altmannshofer, J. Galloway, S. Gori, A.L. Kagan, A. Martin and J. Zupan, $750 \mathrm{GeV}$ diphoton excess, Phys. Rev. D 93 (2016) 095015 [arXiv: 1512.07616] [INSPIRE].

[72] N. Craig, P. Draper, C. Kilic and S. Thomas, Shedding light on diphoton resonances, Phys. Rev. D 93 (2016) 115023 [arXiv: 1512.07733] [InSPIRE].

[73] S. Fichet, G. von Gersdorff and C. Royon, Scattering light by light at $750 \mathrm{GeV}$ at the LHC, Phys. Rev. D 93 (2016) 075031 [arXiv:1512.05751] [InSPIRE].

[74] I. Sahin, Semi-elastic cross section for a scalar resonance of mass $750 \mathrm{GeV}$, arXiv: 1601.01676 [INSPIRE].

[75] S. Fichet, G. von Gersdorff and C. Royon, Measuring the diphoton coupling of a $750 \mathrm{GeV}$ resonance, Phys. Rev. Lett. 116 (2016) 231801 [arXiv:1601.01712] [INSPIRE].

[76] C. Csáki, J. Hubisz, S. Lombardo and J. Terning, Gluon versus photon production of a $750 \mathrm{GeV}$ diphoton resonance, Phys. Rev. D 93 (2016) 095020 [arXiv:1601.00638] [INSPIRE].

[77] L.A. Harland-Lang, V.A. Khoze and M.G. Ryskin, The photon PDF in events with rapidity gaps, Eur. Phys. J. C 76 (2016) 255 [arXiv:1601.03772] [InSPIRE].

[78] L.A. Harland-Lang, V.A. Khoze and M.G. Ryskin, The production of a diphoton resonance via photon-photon fusion, JHEP 03 (2016) 182 [arXiv: 1601.07187] [INSPIRE].

[79] A.D. Martin and M.G. Ryskin, Advantages of exclusive $\gamma \gamma$ production to probe high mass systems, J. Phys. G 43 (2016) 04LT02 [arXiv:1601.07774] [InSPIRE].

[80] L. Bian, N. Chen, D. Liu and J. Shu, Hidden confining world on the $750 \mathrm{GeV}$ diphoton excess, Phys. Rev. D 93 (2016) 095011 [arXiv: 1512.05759] [InSPIRE].

[81] Y. Bai, J. Berger and R. Lu, 750 GeV dark pion: cousin of a dark G-parity odd WIMP, Phys. Rev. D 93 (2016) 076009 [arXiv: 1512.05779] [InSPIRE].

[82] Y. Nakai, R. Sato and K. Tobioka, Footprints of new strong dynamics via anomaly and the $750 \mathrm{GeV}$ diphoton, Phys. Rev. Lett. 116 (2016) 151802 [arXiv: 1512.04924] [INSPIRE].

[83] J.M. No, V. Sanz and J. Setford, See-saw composite Higgs model at the LHC: linking naturalness to the $750 \mathrm{GeV}$ diphoton resonance, Phys. Rev. D 93 (2016) 095010 [arXiv: 1512.05700] [INSPIRE].

[84] D. Bečirević, E. Bertuzzo, O. Sumensari and R. Zukanovich Funchal, Can the new resonance at LHC be a CP-odd Higgs boson?, Phys. Lett. B 757 (2016) 261 [arXiv: 1512.05623] [INSPIRE].

[85] E. Molinaro, F. Sannino and N. Vignaroli, Minimal composite dynamics versus axion origin of the diphoton excess, arXiv:1512.05334 [INSPIRE].

[86] M. Low, A. Tesi and L.-T. Wang, A pseudoscalar decaying to photon pairs in the early LHC Run 2 data, JHEP 03 (2016) 108 [arXiv:1512.05328] [INSPIRE].

[87] I. Ben-Dayan and R. Brustein, Hypercharge axion and the diphoton $750 \mathrm{GeV}$ resonance, arXiv: 1601.07564 [INSPIRE].

[88] A. Pilaftsis, Diphoton signatures from heavy axion decays at the CERN Large Hadron Collider, Phys. Rev. D 93 (2016) 015017 [arXiv:1512.04931] [InSPIRE].

[89] N.D. Barrie, A. Kobakhidze, M. Talia and L. Wu, $750 \mathrm{GeV}$ composite axion as the LHC diphoton resonance, Phys. Lett. B 755 (2016) 343 [arXiv: 1602.00475] [INSPIRE]. 
[90] E. Molinaro, F. Sannino and N. Vignaroli, Collider tests of (composite) diphoton resonances, arXiv: 1602.07574 [INSPIRE].

[91] M. Redi, A. Strumia, A. Tesi and E. Vigiani, Di-photon resonance and dark matter as heavy pions, JHEP 05 (2016) 078 [arXiv: 1602.07297] [INSPIRE].

[92] C.-W. Chiang, H. Fukuda, M. Ibe and T.T. Yanagida, $750 \mathrm{GeV}$ diphoton resonance in a visible heavy QCD axion model, Phys. Rev. D 93 (2016) 095016 [arXiv:1602.07909] [INSPIRE].

[93] A. Bharucha, A. Djouadi and A. Goudelis, Threshold enhancement of diphoton resonances, arXiv: 1603.04464 [INSPIRE].

[94] K. Harigaya and Y. Nomura, Hidden pion varieties in composite models for diphoton resonances, arXiv: 1603.05774 [INSPIRE].

[95] P. Ko, C. Yu and T.-C. Yuan, 750 GeV diphoton excess as a composite (pseudo)scalar boson from new strong interaction, arXiv:1603.08802 [INSPIRE].

[96] M. Chala, C. Grojean, M. Riembau and T. Vantalon, Deciphering the CP nature of the $750 \mathrm{GeV}$ resonance, Phys. Lett. B 760 (2016) 220 [arXiv: 1604.02029] [INSPIRE].

[97] B. Bellazzini, R. Franceschini, F. Sala and J. Serra, Goldstones in diphotons, JHEP 04 (2016) 072 [arXiv: 1512.05330] [inSPIRE].

[98] T. Higaki, K.S. Jeong, N. Kitajima and F. Takahashi, The QCD axion from aligned axions and diphoton excess, Phys. Lett. B 755 (2016) 13 [arXiv:1512.05295] [INSPIRE].

[99] J.E. Kim, Is an axizilla possible for di-photon resonance?, Phys. Lett. B 755 (2016) 190 [arXiv: 1512.08467] [INSPIRE].

[100] L.E. Ibáñez and V. Martin-Lozano, A megaxion at $750 \mathrm{GeV}$ as a first hint of low scale string theory, JHEP 07 (2016) 021 [arXiv:1512.08777] [INSPIRE].

[101] K. Ghorbani and H. Ghorbani, The $750 \mathrm{GeV}$ diphoton excess from a pseudoscalar in fermionic dark matter scenario, arXiv:1601.00602 [INSPIRE].

[102] D. Stolarski and R. Vega-Morales, Probing a virtual diphoton excess, Phys. Rev. D 93 (2016) 055008 [arXiv: 1601.02004] [INSPIRE].

[103] U. Ellwanger and C. Hugonie, A $750 \mathrm{GeV}$ diphoton signal from a very light pseudoscalar in the NMSSM, JHEP 05 (2016) 114 [arXiv:1602.03344] [INSPIRE].

[104] S. Di Chiara, A. Hektor, K. Kannike, L. Marzola and M. Raidal, Large loop-coupling enhancement of a $750 \mathrm{GeV}$ pseudoscalar from a light dark sector, arXiv:1603.07263 [INSPIRE].

[105] T. Gherghetta, N. Nagata and M. Shifman, A visible QCD axion from an enlarged color group, Phys. Rev. D 93 (2016) 115010 [arXiv:1604.01127] [INSPIRE].

[106] P. Lebiedowicz, M. Luszczak, R. Pasechnik and A. Szczurek, Can the diphoton enhancement at $750 \mathrm{GeV}$ be due to a neutral technipion?, Phys. Rev. D 94 (2016) 015023 [arXiv: 1604. 02037] [INSPIRE].

[107] A. Kusenko, L. Pearce and L. Yang, Leptogenesis via the 750 GeV pseudoscalar, Phys. Rev. D 93 (2016) 115005 [arXiv: 1604.02382] [INSPIRE].

[108] ATLAS collaboration, Search for new phenomena in the dilepton final state using proton-proton collisions at $\sqrt{s}=13 \mathrm{TeV}$ with the ATLAS detector, ATLAS-CONF-2015-070, CERN, Geneva Switzerland (2015). 
[109] ATLAS collaboration, Search for neutral minimal supersymmetric Standard Model Higgs bosons $H / A \rightarrow \tau \tau$ produced in pp collisions at $\sqrt{s}=13 \mathrm{TeV}$ with the ATLAS detector, ATLAS-CONF-2015-061, CERN, Geneva Switzerland (2015).

[110] ATLAS collaboration, Search for heavy resonances decaying to a $Z$ boson and a photon in pp collisions at $\sqrt{s}=13 \mathrm{TeV}$ with the ATLAS detector, ATLAS-CONF-2016-010, CERN, Geneva Switzerland (2016).

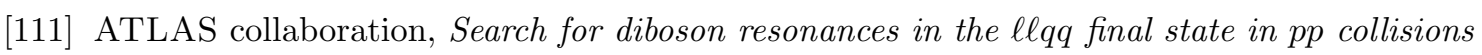
at $\sqrt{s}=13 \mathrm{TeV}$ with the ATLAS detector, ATLAS-CONF-2015-071, CERN, Geneva Switzerland (2015).

[112] ATLAS collaboration, Search for new resonances decaying to a $W$ or $Z$ boson and a Higgs boson in the $\ell \ell b \bar{b}, \ell \nu b \bar{b}$ and $\nu \nu b \bar{b}$ channels in pp collisions at $\sqrt{s}=13$ TeV with the ATLAS detector, ATLAS-CONF-2015-074, CERN, Geneva Switzerland (2015).

[113] ATLAS collaboration, Search for pair production of Higgs bosons in the $b \bar{b} b \bar{b}$ final state using proton-proton collisions at $\sqrt{s}=13 \mathrm{TeV}$ with the ATLAS detector, ATLAS-CONF-2016-017, CERN, Geneva Switzerland (2016).

[114] ATLAS collaboration, Search for $W W / W Z$ resonance production in the $\ell \nu q q$ final state at $\sqrt{s}=13 \mathrm{TeV}$ with the ATLAS detector at the LHC, ATLAS-CONF-2015-075, CERN, Geneva Switzerland (2015).

[115] ATLAS collaboration, Search for new phenomena in final states with an energetic jet and large missing transverse momentum in pp collisions at $\sqrt{s}=13$ TeV using the ATLAS detector, arXiv:1604.07773 [INSPIRE].

[116] NNPDF collaboration, R.D. Ball et al., Parton distributions for the LHC Run 2, JHEP 04 (2015) 040 [arXiv: 1410.8849] [INSPIRE].

[117] J. Alwall et al., The automated computation of tree-level and next-to-leading order differential cross sections and their matching to parton shower simulations, JHEP 07 (2014) 079 [arXiv: 1405.0301] [INSPIRE].

[118] M. Bauer, C. Hoerner and M. Neubert, Diphoton resonance from a warped extra dimension, arXiv: 1603.05978 [INSPIRE].

[119] R. Alonso, E.E. Jenkins and A.V. Manohar, Holomorphy without supersymmetry in the Standard Model effective field theory, Phys. Lett. B 739 (2014) 95 [arXiv:1409.0868] [INSPIRE].

[120] J. Elias-Miro, J.R. Espinosa and A. Pomarol, One-loop non-renormalization results in EFTs, Phys. Lett. B $\mathbf{7 4 7}$ (2015) 272 [arXiv:1412.7151] [INSPIRE].

[121] C. Cheung and C.-H. Shen, Nonrenormalization theorems without supersymmetry, Phys. Rev. Lett. 115 (2015) 071601 [arXiv:1505.01844] [INSPIRE].

[122] B. Gripaios and D. Sutherland, An operator basis for the Standard Model with an added scalar singlet, arXiv:1604.07365 [INSPIRE].

[123] G. D'Ambrosio, G.F. Giudice, G. Isidori and A. Strumia, Minimal flavor violation: an effective field theory approach, Nucl. Phys. B 645 (2002) 155 [hep-ph/0207036] [INSPIRE].

[124] P. Ciafaloni, D. Comelli, A. Riotto, F. Sala, A. Strumia and A. Urbano, Weak corrections are relevant for dark matter indirect detection, JCAP 03 (2011) 019 [arXiv: 1009. 0224] [INSPIRE]. 
[125] R.P. Kauffman, S.V. Desai and D. Risal, Production of a Higgs boson plus two jets in hadronic collisions, Phys. Rev. D 55 (1997) 4005 [Erratum ibid. D 58 (1998) 119901] [hep-ph/9610541] [INSPIRE].

[126] R.P. Kauffman, S.V. Desai and D. Risal, Amplitudes for Higgs bosons plus four partons, hep-ph/9903330 [INSPIRE].

[127] L.J. Dixon, E.W.N. Glover and V.V. Khoze, MHV rules for Higgs plus multi-gluon amplitudes, JHEP 12 (2004) 015 [hep-th/0411092] [INSPIRE].

[128] K. Hagiwara, Q. Li and K. Mawatari, Jet angular correlation in vector-boson fusion processes at hadron colliders, JHEP 07 (2009) 101 [arXiv:0905.4314] [INSPIRE].

[129] A.R. Barker, H. Huang, P.A. Toale and J. Engle, Radiative corrections to double Dalitz decays: effects on invariant mass distributions and angular correlations, Phys. Rev. D 67 (2003) 033008 [hep-ph/0210174] [INSPIRE].

[130] KTEV collaboration, E. Abouzaid et al., Determination of the parity of the neutral pion via the four-electron decay, Phys. Rev. Lett. 100 (2008) 182001 [arXiv:0802.2064] [INSPIRE].

[131] R.K. Ellis, I. Hinchliffe, M. Soldate and J.J. van der Bij, Higgs decay to $\tau^{+} \tau^{-}$: a possible signature of intermediate mass Higgs bosons at the SSC, Nucl. Phys. B 297 (1988) 221 [INSPIRE].

[132] G. Klamke and D. Zeppenfeld, Higgs plus two jet production via gluon fusion as a signal at the CERN LHC, JHEP 04 (2007) 052 [hep-ph/0703202] [inSPIRE].

[133] J.R. Andersen, K. Arnold and D. Zeppenfeld, Azimuthal angle correlations for Higgs boson plus multi-jet events, JHEP 06 (2010) 091 [arXiv: 1001.3822] [INSPIRE].

[134] C. Englert, M. Spannowsky and M. Takeuchi, Measuring Higgs CP and couplings with hadronic event shapes, JHEP 06 (2012) 108 [arXiv: 1203.5788] [INSPIRE].

[135] K. Cranmer, B. Mellado, W. Quayle and S.L. Wu, Search for Higgs bosons decay $H \rightarrow \gamma \gamma$ using vector boson fusion, hep-ph/0401088 [INSPIRE].

[136] F. James, Statistical methods in experimental physics, World Scientific, Hackensack U.S.A. (2006) [INSPIRE].

[137] A.D. Martin, W.J. Stirling, R.S. Thorne and G. Watt, Parton distributions for the LHC, Eur. Phys. J. C 63 (2009) 189 [arXiv:0901.0002] [INSPIRE].

[138] CMS collaboration, Search for neutral resonances decaying into a $Z$ boson and a pair of $b$ jets or $\tau$ leptons, Phys. Lett. B 759 (2016) 369 [arXiv: 1603. 02991] [INSPIRE].

[139] I. Anderson et al., Constraining anomalous $H V V$ interactions at proton and lepton colliders, Phys. Rev. D 89 (2014) 035007 [arXiv: 1309.4819] [INSPIRE].

[140] L. Di Luzio, J.F. Kamenik and M. Nardecchia, Implications of perturbative unitarity for the $\gamma \gamma$ resonance at $750 \mathrm{GeV}$, arXiv: 1604.05746 [INSPIRE].

[141] CMS collaboration, Search for pair-produced resonances decaying to jet pairs in proton-proton collisions at $\sqrt{s}=8 \mathrm{TeV}$, Phys. Lett. B 747 (2015) 98 [arXiv:1412.7706] [INSPIRE].

[142] ATLAS collaboration, Search for new phenomena in events with at least three photons collected in pp collisions at $\sqrt{s}=8 \mathrm{TeV}$ with the ATLAS detector, Eur. Phys. J. C 76 (2016) 210 [arXiv: 1509.05051] [INSPIRE]. 
[143] ATLAS collaboration, Analysis of events with b-jets and a pair of leptons of the same charge in pp collisions at $\sqrt{s}=8 \mathrm{TeV}$ with the ATLAS detector, JHEP 10 (2015) 150 [arXiv: 1504.04605] [INSPIRE].

[144] J.R. Ellis, M.K. Gaillard and D.V. Nanopoulos, A phenomenological profile of the Higgs boson, Nucl. Phys. B 106 (1976) 292 [InSPIRE].

[145] M.A. Shifman, A.I. Vainshtein, M.B. Voloshin and V.I. Zakharov, Low-energy theorems for Higgs boson couplings to photons, Sov. J. Nucl. Phys. 30 (1979) 711 [Yad. Fiz. 30 (1979) 1368] [INSPIRE].

[146] A.I. Vainshtein, V.I. Zakharov and M.A. Shifman, Higgs particles, Sov. Phys. Usp. 23 (1980) 429 [Usp. Fiz. Nauk 131 (1980) 537] [INSPIRE].

[147] M.B. Voloshin, Once again about the role of gluonic mechanism in interaction of light Higgs boson with hadrons, Sov. J. Nucl. Phys. 44 (1986) 478 [Yad. Fiz. 44 (1986) 738] [InSPIRE].

[148] M.A. Shifman, Anomalies and low-energy theorems of quantum chromodynamics, Phys. Rept. 209 (1991) 341 [Sov. Phys. Usp. 32 (1989) 289] [Usp. Fiz. Nauk 157 (1989) 561] [INSPIRE].

[149] J.F. Gunion, H.E. Haber, G.L. Kane and S. Dawson, The Higgs hunter's guide, Front. Phys. 80 (2000) 1 [INSPIRE].

[150] B.A. Kniehl and M. Spira, Low-energy theorems in Higgs physics, Z. Phys. C 69 (1995) 77 [hep-ph/9505225] [INSPIRE].

[151] E.W.N. Glover and J.J. van der Bij, Higgs boson pair production via gluon fusion, Nucl. Phys. B 309 (1988) 282 [INSPIRE].

[152] T. Plehn, M. Spira and P.M. Zerwas, Pair production of neutral Higgs particles in gluon-gluon collisions, Nucl. Phys. B 479 (1996) 46 [Erratum ibid. B 531 (1998) 655] [hep-ph/9603205] [INSPIRE].

[153] S. Dawson, S. Dittmaier and M. Spira, Neutral Higgs boson pair production at hadron colliders: QCD corrections, Phys. Rev. D 58 (1998) 115012 [hep-ph/9805244] [INSPIRE].

[154] A. Djouadi, W. Kilian, M. Muhlleitner and P.M. Zerwas, Production of neutral Higgs boson pairs at LHC, Eur. Phys. J. C 10 (1999) 45 [hep-ph/9904287] [INSPIRE].

[155] U. Baur, T. Plehn and D.L. Rainwater, Measuring the Higgs boson self coupling at the LHC and finite top mass matrix elements, Phys. Rev. Lett. 89 (2002) 151801 [hep-ph/0206024] [INSPIRE].

[156] E. Del Nobile, R. Franceschini, D. Pappadopulo and A. Strumia, Minimal matter at the Large Hadron Collider, Nucl. Phys. B 826 (2010) 217 [arXiv:0908.1567] [InSPIRE].

[157] R. Franceschini and R. Torre, RPV stops bump off the background, Eur. Phys. J. C 73 (2013) 2422 [arXiv: 1212.3622] [INSPIRE].

[158] F. Bishara, Y. Grossman, R. Harnik, D.J. Robinson, J. Shu and J. Zupan, Probing CP-violation in $h \rightarrow \gamma \gamma$ with converted photons, JHEP 04 (2014) 084 [arXiv:1312.2955] [INSPIRE].

[159] A. Djouadi, The anatomy of electro-weak symmetry breaking. I: the Higgs boson in the Standard Model, Phys. Rept. 457 (2008) 1 [hep-ph/0503172] [InSPIRE]. 